\title{
ALTERNATIVE MISSION CONCEPTS FOR THE EXPLORATION OF OUTER PLANETS USING SMALL
} SATELLITE SWARMS

\author{
A Thesis \\ presented to \\ the Faculty of California Polytechnic State University, \\ San Luis Obispo
}

In Partial Fulfillment

of the Requirements for the Degree

Master of Science in Aerospace Engineering

by

Andrew Gene Blocher

November 2017 
(C) 2017

Andrew Gene Blocher

ALL RIGHTS RESERVED 


\section{COMMITTEE MEMBERSHIP}

TITLE: Alternative Mission Concepts for the Exploration of Outer Planets Using Small Satellite Swarms

AUTHOR: $\quad$ Andrew Gene Blocher

DATE SUBMITTED: $\quad$ November 2017

COMMITTEE CHAIR: $\quad$ Dr. Jordi Puig-Suari, Ph.D.

Professor of Aerospace Engineering

COMMITTEE MEMBER: Dr. Kira Abercromby, Ph.D.

Associate Professor of Aerospace Engineering

COMMITTEE MEMBER: $\quad$ Dr. Amelia Greig, Ph.D.

Assistant Professor of Aerospace Engineering

COMMITTEE MEMBER: $\quad$ Dr. Anthony Freeman, Ph.D.

Systems Engineer Jet Propulsion Laboratory 


\begin{abstract}
Alternative Mission Concepts for the Exploration of Outer Planets Using Small Satellite Swarms
\end{abstract}

Andrew Gene Blocher

Interplanetary space exploration has thus far consisted of single, expensive spacecraft missions. Mission costs are particularly high on missions to the outer planets and while invaluable, finite budgets limit our ability to perform extensive and frequent investigations of the planets. Planetary systems such as Jupiter and Saturn provide extremely complex exploration environments with numerous targets of interest. Exploring these targets in addition to the main planet requires multiple fly-bys and long mission timelines.

In LEO, CubeSats have changed the exploration paradigm, offering a fast and low cost alternative to traditional space vehicles. This new mission development philosophy has the potential to significantly change the economics of interplanetary exploration and a number of missions are being developed to utilize CubeSat class spacecraft beyond earth orbit (e.g., NEAScout, Lunar Ice Cube, Marco and BioSentinel).

This paper takes the CubeSat philosophical approach one step further by investigating the potential for small satellite swarms to provide extensive studies of the Saturn system. To do this, an architecture was developed to best replicate the Cassini Primary Mission science objectives using swarms of CubeSats. Cassini was chosen because of its complexity and it defines a well-understood baseline to compare against. The paper outlines the overall mission architecture developed and provides a feasible initial design for the spacecraft in the architecture. The number of swarms needed, number of CubeSats per swarm, size of the CubeSats, overall science output and estimated mission cost are all presented. Additional science objectives beyond Cassini's capabilities are also proposed.

Significant scientific returns can be achieved by the swarm based architecture and the risk tolerance afforded by the utilization of large numbers of low-cost sensor carriers. This study found a potential architecture that could reduce the cost of replicating Cassini by as much as 63\%. The results of this investigation are not constrained to Saturn and can be easily translated to other targets such as Uranus, Neptune or the asteroid belt.

Keywords: Cassini, CubeSat, NanoSat, SmallSat, Swarm, Saturn, Titan, Enceladus, Spacecraft, Architecture, Interplanetary, Deep, Space 


\section{ACKNOWLEDGMENTS}

There are many people involved in my college career and thesis process. I'd like to thank some of them here.

My advisor Dr. Puig-Suari for presenting me with this thesis idea and helping me through

Dr. Tony Freeman for being one of my advisors and hosting me at JPL for the summer where I worked on my thesis and fulfilled a lifelong dream of working at a NASA center.

Kendra, the Aerospace Department Secretary, is someone I will be forever indebted to for helping me through my college career and many paperwork nightmares.

My girlfriend, Emily for pushing me through the final stage for my Masters degree and college career, and also for feeding me.

Shane "The Train" Sheehan, roommate, thesis buddy and friend.

Last. but not least, I'd like to thank my parents for enabling and supporting me to pursue my dreams.

Thank you all. 
LIST OF TABLES viii

LIST OF FIGURES . ix

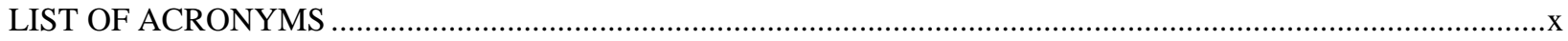

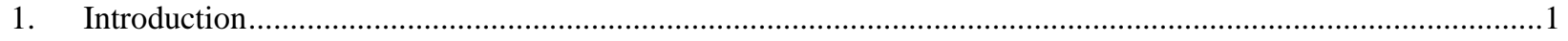

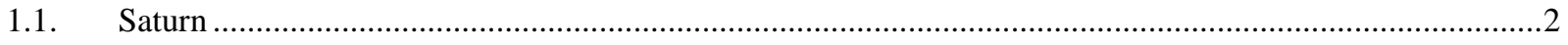

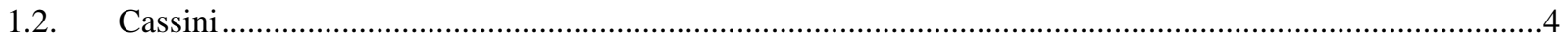

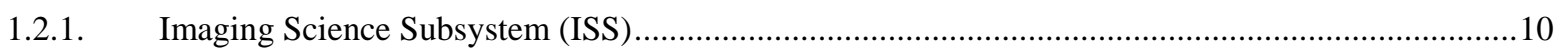

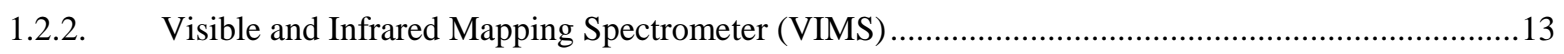

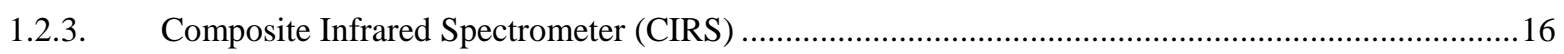

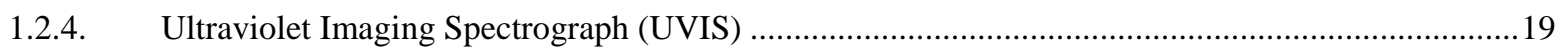

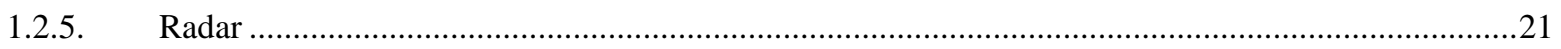

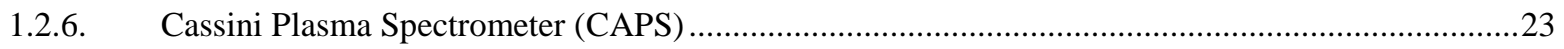

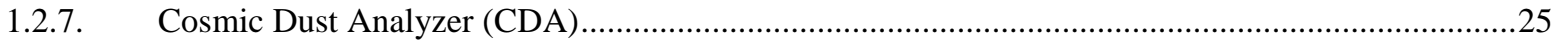

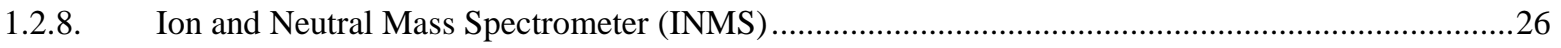

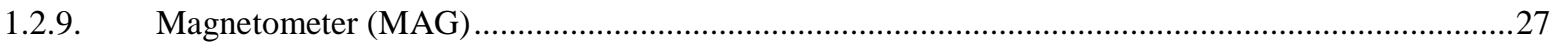

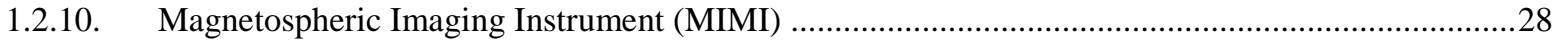

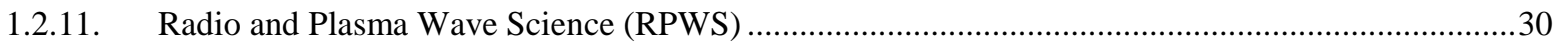

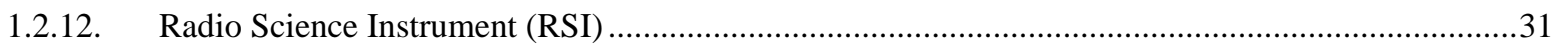

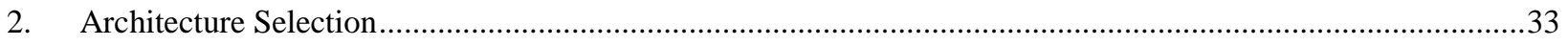

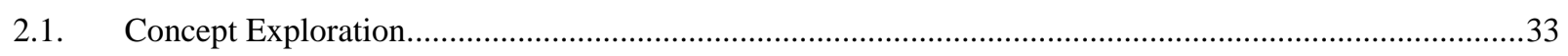

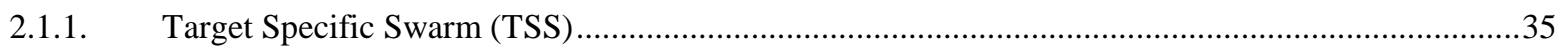

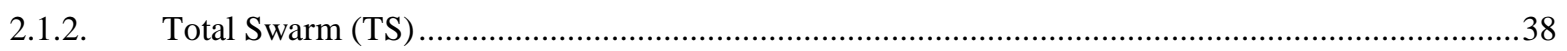

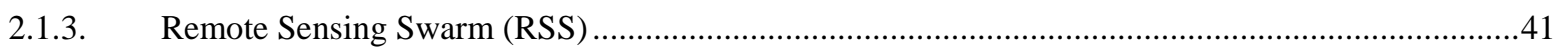

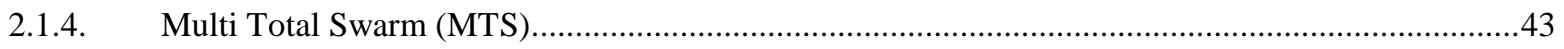

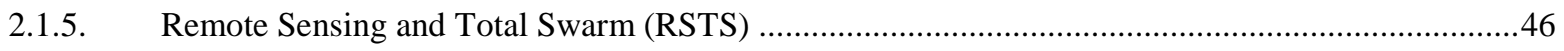

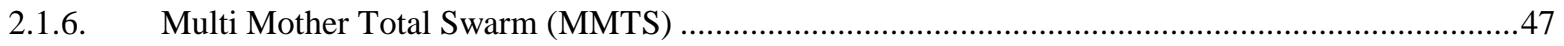

2.1.7. Multi Mother Remote Sensing Swarm (MMRSS) …..........................................................49

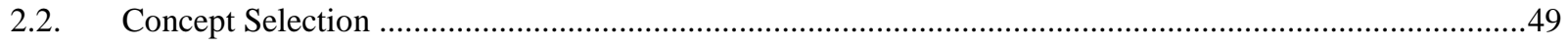

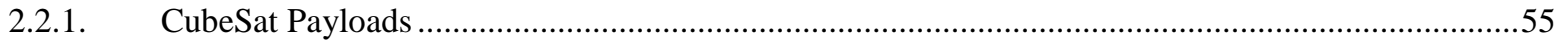

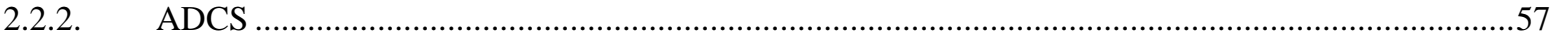

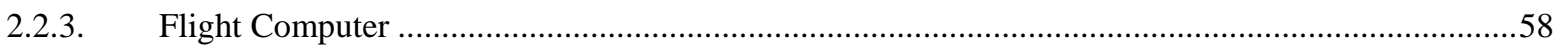

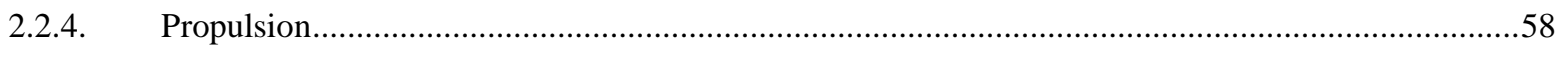

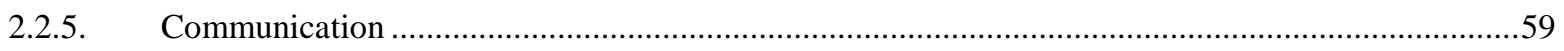




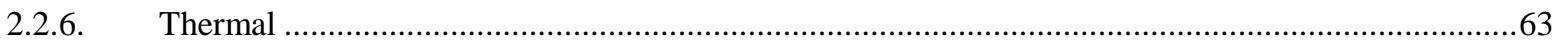

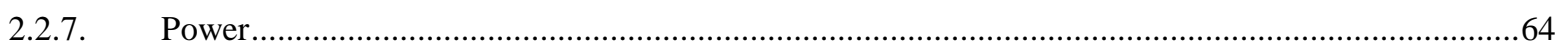

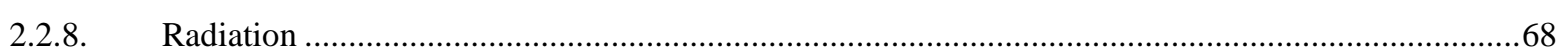

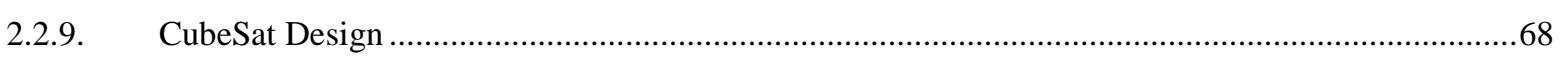

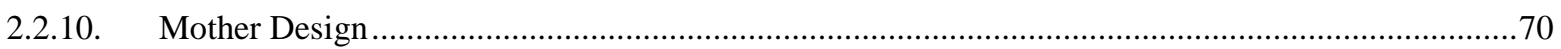

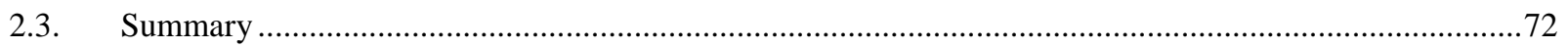

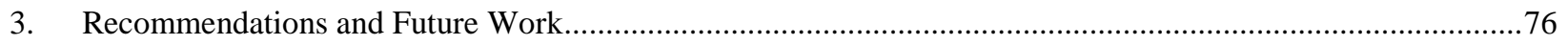

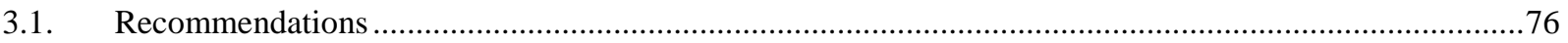

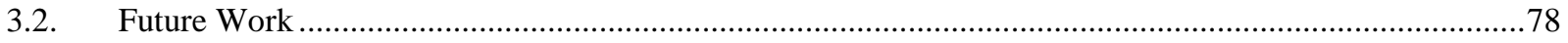

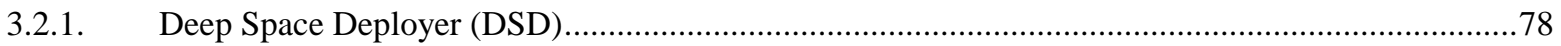

3.2.2. Trajectory Analysis and Optimization........................................................................................... 78

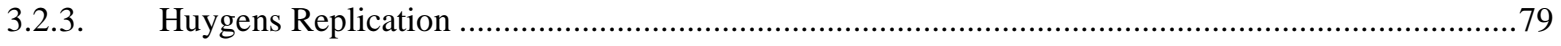

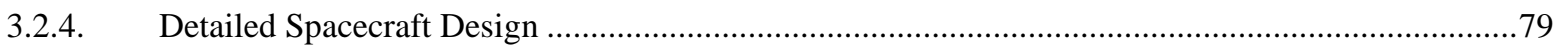

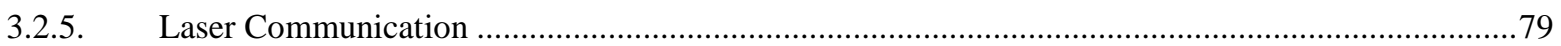

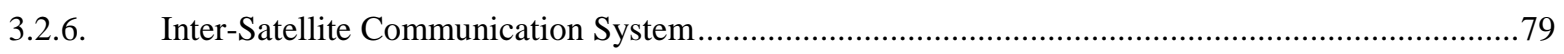

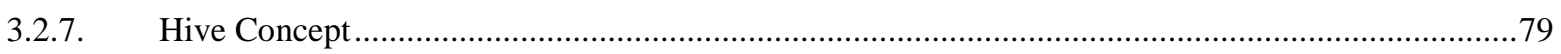

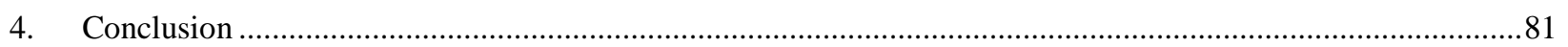

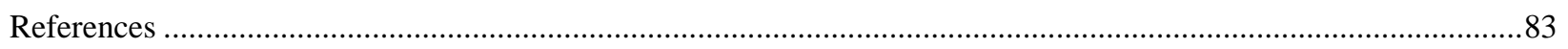

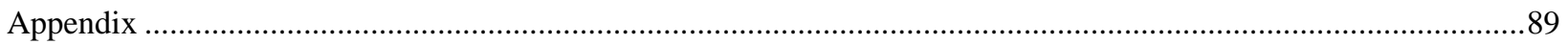




\section{LIST OF TABLES}

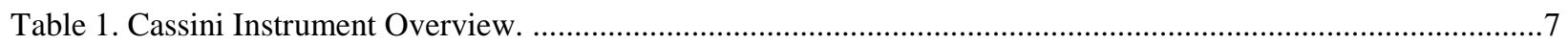

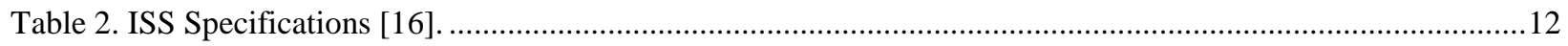

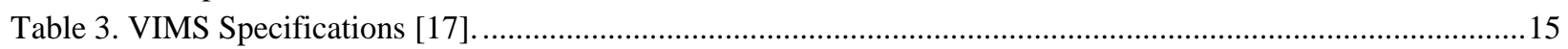

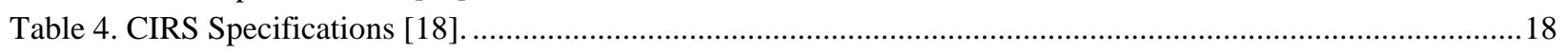

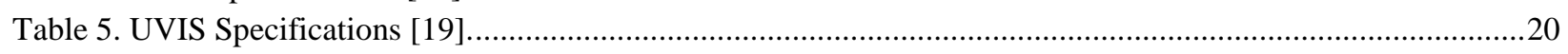

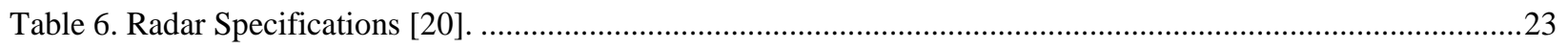

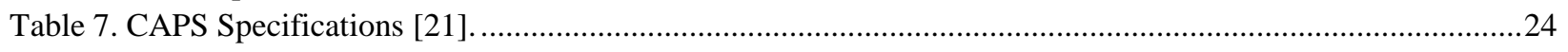

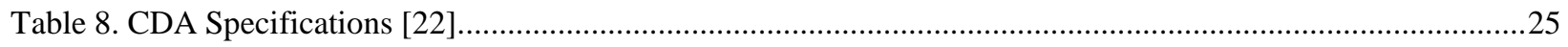

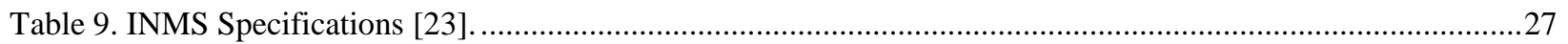

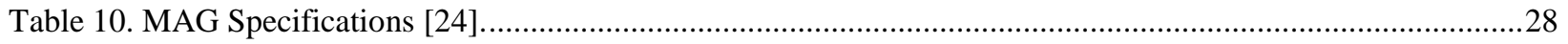

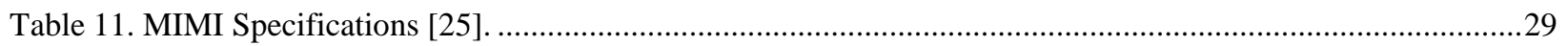

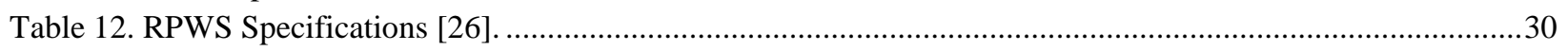

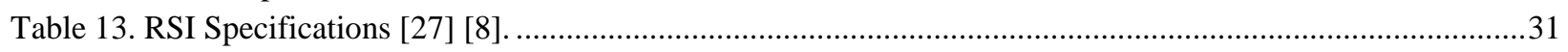

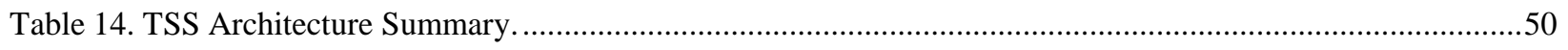

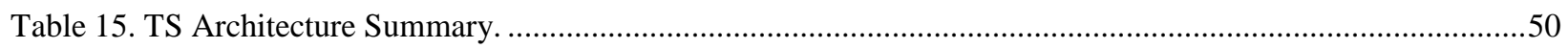

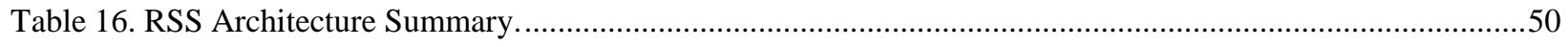

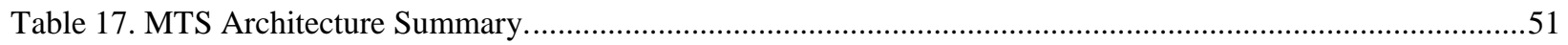

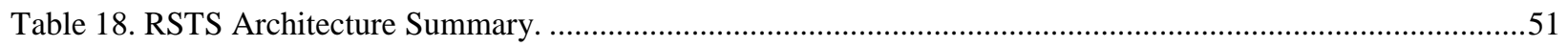

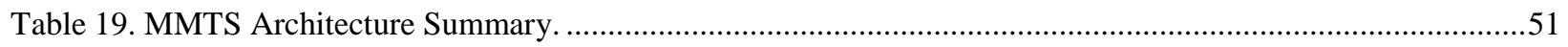

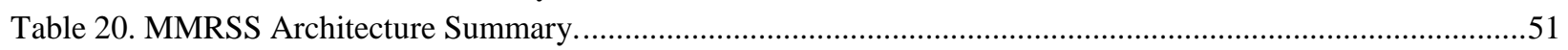

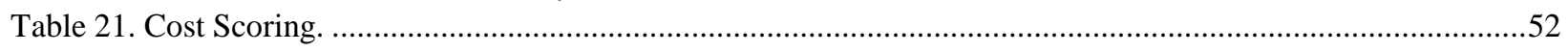

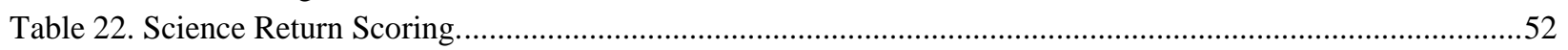

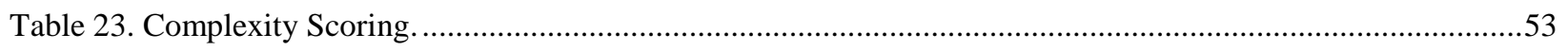

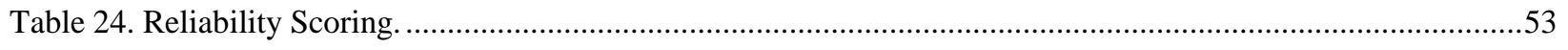

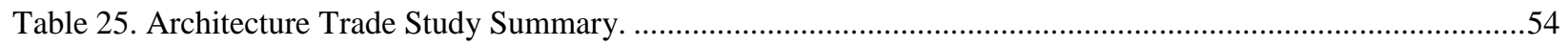

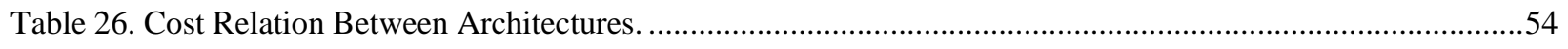

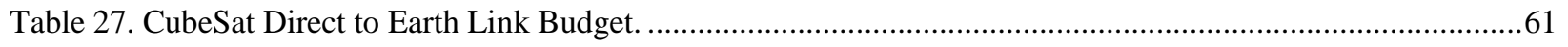

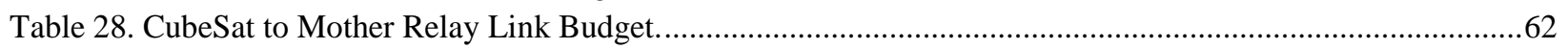

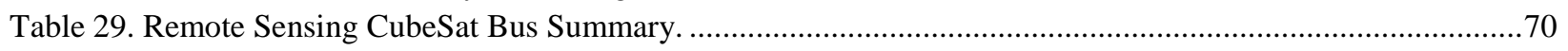

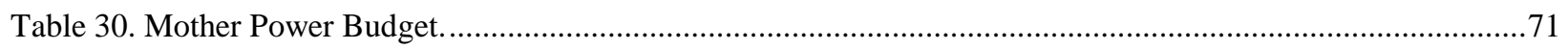




\section{LIST OF FIGURES}

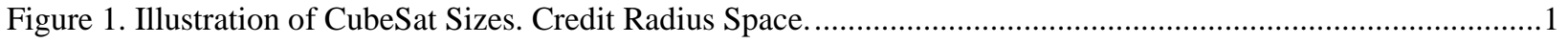

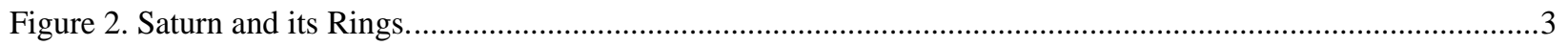

Figure 3. Diagram of Saturn's Rings and Important Moons. .......................................................................

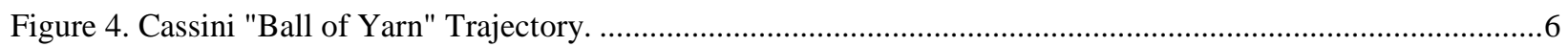

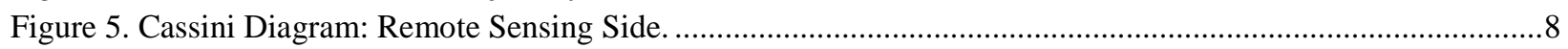

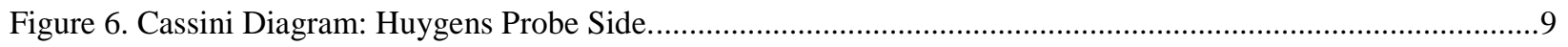

Figure 7. Cassini ISS. The NAC is on top with the WAC on bottom.......................................................11

Figure 8. Cassini VIMS Instrument with VIMS-VIS bottom left and VIMS-IR top right. ....................................14

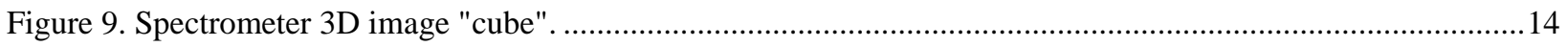

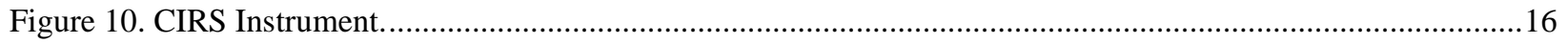

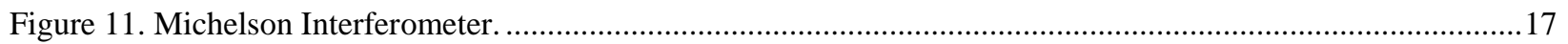

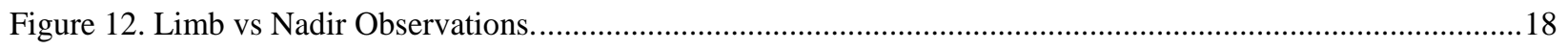

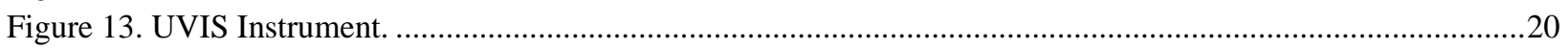

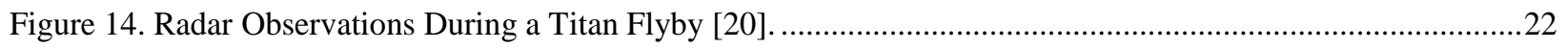

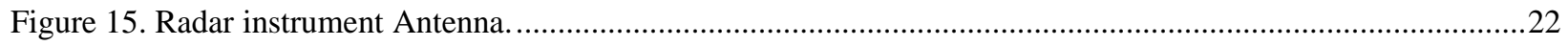

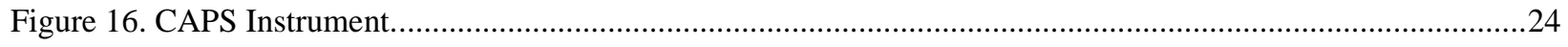

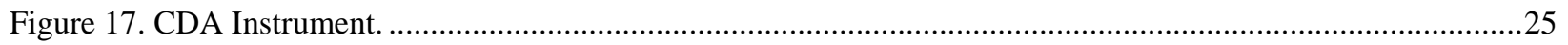

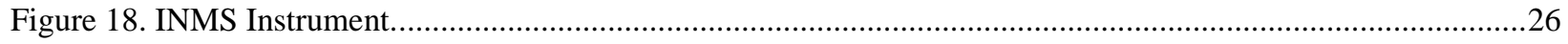

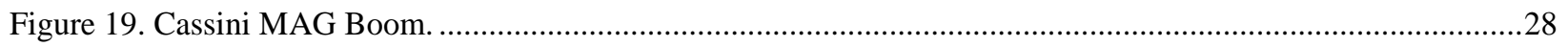

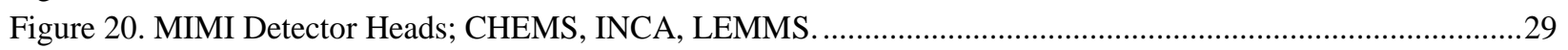

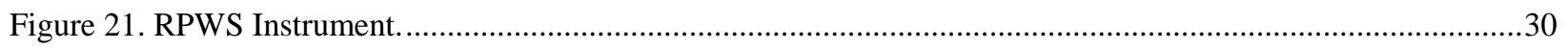

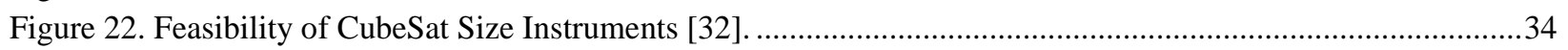

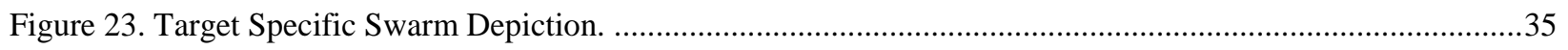

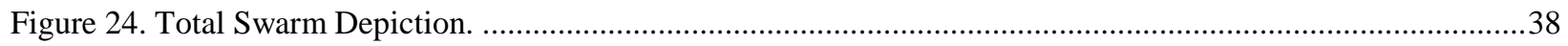

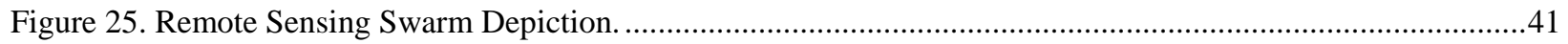

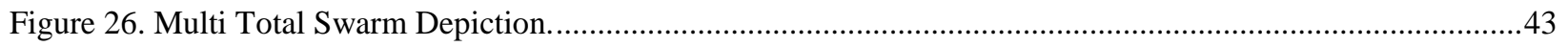

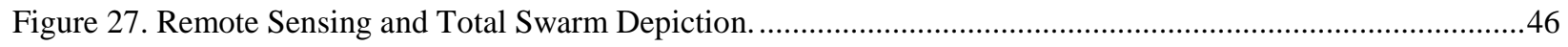

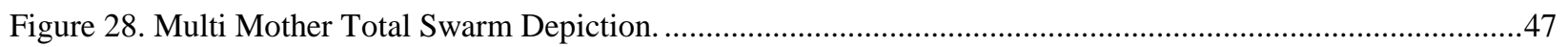

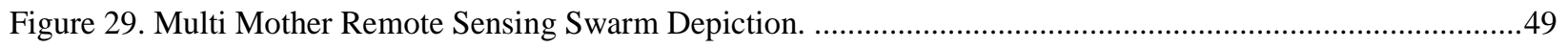

Figure 30. XACT 50 ADCS. Credit Blue Canyon Technologies. .........................................................................57

Figure 31. Leon 3 Sphinx Computer. Credit Jet Propulsion Laboratory. ...............................................................58

Figure 32. Aerojet Rocketdyne CubeSat Propulsion System............................................................................59

Figure 33. Iris Deep Space Transponder. Credit Jet Propulsion Laboratory. ...................................................60

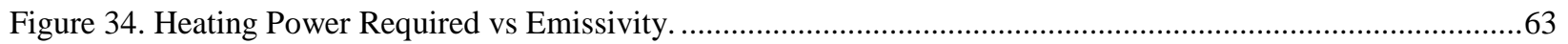

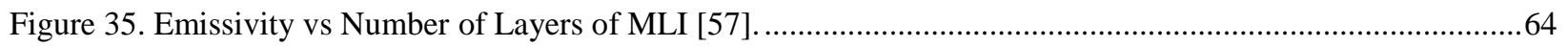

Figure 36. Lunar IceCube. Credit Morehead State University. .......................................................................65

Figure 37. Time to Recharge Batteries vs Power Consumed.............................................................................66

Figure 38. Total dose of the proposed Prometheus Saturn Moon Mission which had a four year minimum mission

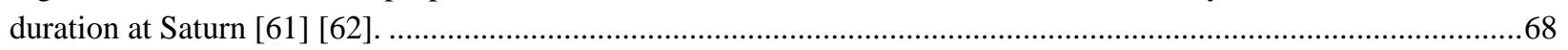

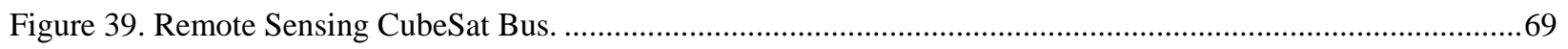

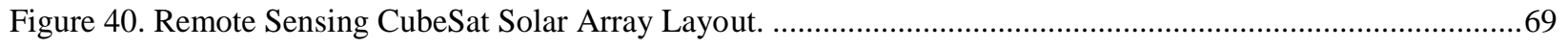

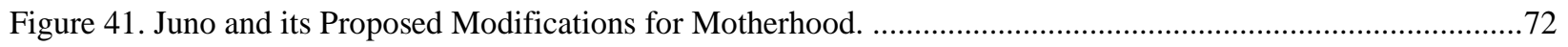

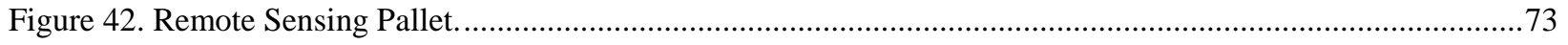




\section{LIST OF ACRONYMS}

\begin{tabular}{|c|c|}
\hline $\mathrm{AU}$ & Astronomical Unit \\
\hline CAPS & Cassini Plasma Spectrometer \\
\hline CCD & Charge Coupled Device \\
\hline CDA & Cosmic Dust Analyzer \\
\hline CHEMS & Charge Energy Mass Spectrometer \\
\hline CIRS & Composite Infrared Spectrometer \\
\hline COTS & Commercial Off The Shelf \\
\hline DSN & Deep Space Network \\
\hline ELS & Electron Spectrometer \\
\hline FGM & Fluxgate Magnetometer \\
\hline GEO & Geosynchronous Earth Orbit \\
\hline HDAC & Hydrogen Deuterium Absorption Cell \\
\hline HSP & High Speed Photometer \\
\hline IBS & Ion Beam Spectrometer \\
\hline IMS & Ion Mass Spectrometer \\
\hline INCA & Ion and Neutral Camera \\
\hline INMS & Ion and Neutral Mass Spectrometer \\
\hline IR & Infrared \\
\hline ISS & Imaging Science Subsystem \\
\hline JPL & Jet Propulsion Laboratory \\
\hline LEMMS & Low Energy Magnetospheric Measurements System \\
\hline LEO & Low Earth Orbit \\
\hline MAG & Magnetometer \\
\hline NAC & Narrow Angle Camera \\
\hline NASA & National Aeronautics and Space Administration \\
\hline NIR & Near Infrared \\
\hline
\end{tabular}




\begin{tabular}{|l|l|}
\hline RF & Radio Frequency \\
\hline RPWS & Radio and Plasma Wave Science \\
\hline RSI & Radio Science Instrument \\
\hline SAR & Synthetic Aperture Radar \\
\hline SHM & Scalar Helium Magnetometer \\
\hline SOI & Saturn Orbit Insertion \\
\hline SWAP & Size Weight And Power \\
\hline SWIR & Short Wave Infrared \\
\hline UV & Ultraviolet \\
\hline UVIS & Ultraviolet Imaging Spectrometer \\
\hline VHM & Vector Helium Magnetometer \\
\hline VIMS & Visible and Infrared Mapping Spectrometer \\
\hline VIS & Visible \\
\hline WAC & Wide Angle Camera \\
\hline
\end{tabular}




\section{Introduction}

This thesis aims to determine the ability of small satellite swarms to perform flagship class missions. Recent technological advances have made small satellites increasingly capable of performing high level science. To assess the feasibility of small satellites to perform flagship missions, this thesis will attempt to replicate the Cassini Primary mission. The Cassini Primary mission which lasted from 2004 - 2008 was a complex mission performing 45 flybys of Titan and 7 flybys of other moon all while orbiting Saturn and carrying 12 instruments to investigate the entire Saturn system. The Cassini Primary mission offers an excellent baseline against which various small satellite swarm architectures can be compared. This thesis will do just that by developing several architectures and evaluating the best one to replicate Cassini as well as offering an initial design of the architecture to determine its technical feasibility.

Much of the recent advancement has been focused on a class of spacecraft known as CubeSats. For this reason, only CubeSats will be considered for the architectures proposed. CubeSats conform to the CubeSat standard and have a base size of one unit, or $1 \mathrm{U}$, that is $10 \times 10 \times 10 \mathrm{~cm}$. Multiple Us can be put together to form larger spacecraft with common sizes of 2, 3, 6 and 12U as shown in Figure 1. CubeSats are housed within enclosed deployers that eject the CubeSat upon command. The deployer has all the mechanical and vibrational strength and has been preapproved to be attached to the primary vehicle allowing the CubeSat to have a much less rigorous design as long as it fits inside the deployer and meets the interface requirements. The standard has allowed CubeSats to launch cheaply as secondary payloads and opened the door to many organizations and universities to build and launch CubeSats that would otherwise not have been able to afford a traditional spacecraft.

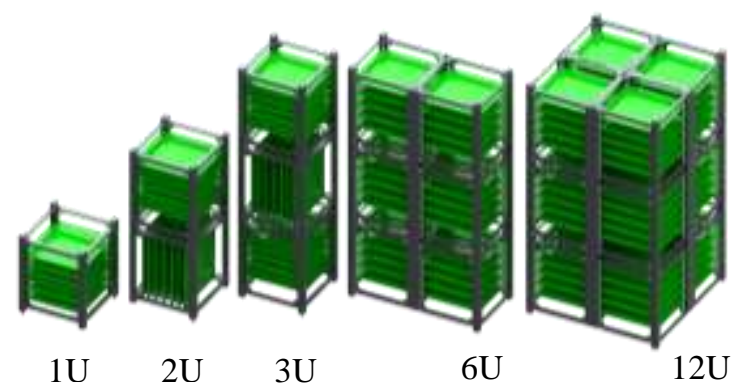

Figure 1. Illustration of CubeSat Sizes. Credit Radius Space. 
Flagship missions are NASA's most expensive class of mission typically costing over $\$ 1$ billion. The total cost of the Cassini mission through the Primary mission was $\$ 3.26$ billion in the year 2000's dollars [1] which translates to $\$ 4.73$ billion in today's dollars [2]. The cost was separated into $\$ 1.4$ billion for prelaunch development such as engineering and spacecraft construction, \$704 million for spacecraft operation, $\$ 54$ million for tracking and navigation assistance from the DSN, and \$422 million for the launch vehicle. The cost was split among several space organizations with the U.S. paying $\$ 2.6$ billion, the European Space Agency contributing \$500 million and the Italian Space Agency contributing \$160 million [1]. Assuming the European Space Agency and Italian contributions were solely for the Huygens Probe, the cost of the Cassini Orbiter Mission would be $\$ 3.95$ billion in today’s dollars.

CubeSats, with their low cost, could offer a low-cost alternative to monolithic spacecraft typically used for flagship missions. The 2017 NASA budget for space exploration is $\$ 4.33$ billion which comprises all of NASA's exploration activities. Even if the cost of Cassini was spread out over many years, it would still have a large impact on other programs. Reducing the cost of flagship missions would allow NASA to fly more missions, increasing our knowledge of the solar system. CubeSats also offer other benefits such as increasing spatial and temporal resolutions by collecting data with multiple CubeSats or performing higher risk measurements knowing there is another CubeSat to take its place should it fail.

No CubeSat has ever flown in interplanetary space, let alone a swarm of CubeSats [3]. This thesis seeks to determine the ideal architecture for implementing CubeSats to replicate the Cassini Primary mission, with the ultimate goal of reducing mission costs. Attention will be paid to how the small CubeSats effect architecture design and what the swarm would look like to achieve the science goals of Cassini. Initial CubeSat designs will be offered to investigate their technical feasibility and illustrate what a deep space CubeSat would require to function. A swarm successful at replicating Cassini with a reduced cost that is technically feasible could provide a baseline architecture to implement on future flagship exploration missions.

\subsection{Saturn}

Saturn is the second largest planet in our solar system behind Jupiter having a mass of $568.3 \times 10^{24} \mathrm{~kg}$ and an equatorial radius of $60,268 \mathrm{~km}$. Saturn's semimajor axis $9.58 \mathrm{AU}$, or $1.434 \times 10^{9} \mathrm{~km}$ from the sun with 
one Saturn completing one orbit every 29.46 years. Saturn is one of four gas giants in our solar system with the other three being Jupiter, Uranus and Neptune. Saturn has 53 named moons [4] with the largest and most famous being Titan. Titan is the only moon known moon in the solar system to have a thick atmosphere and is larger than the planet Mercury with a mean radius of $2575 \mathrm{~km}$ [5].

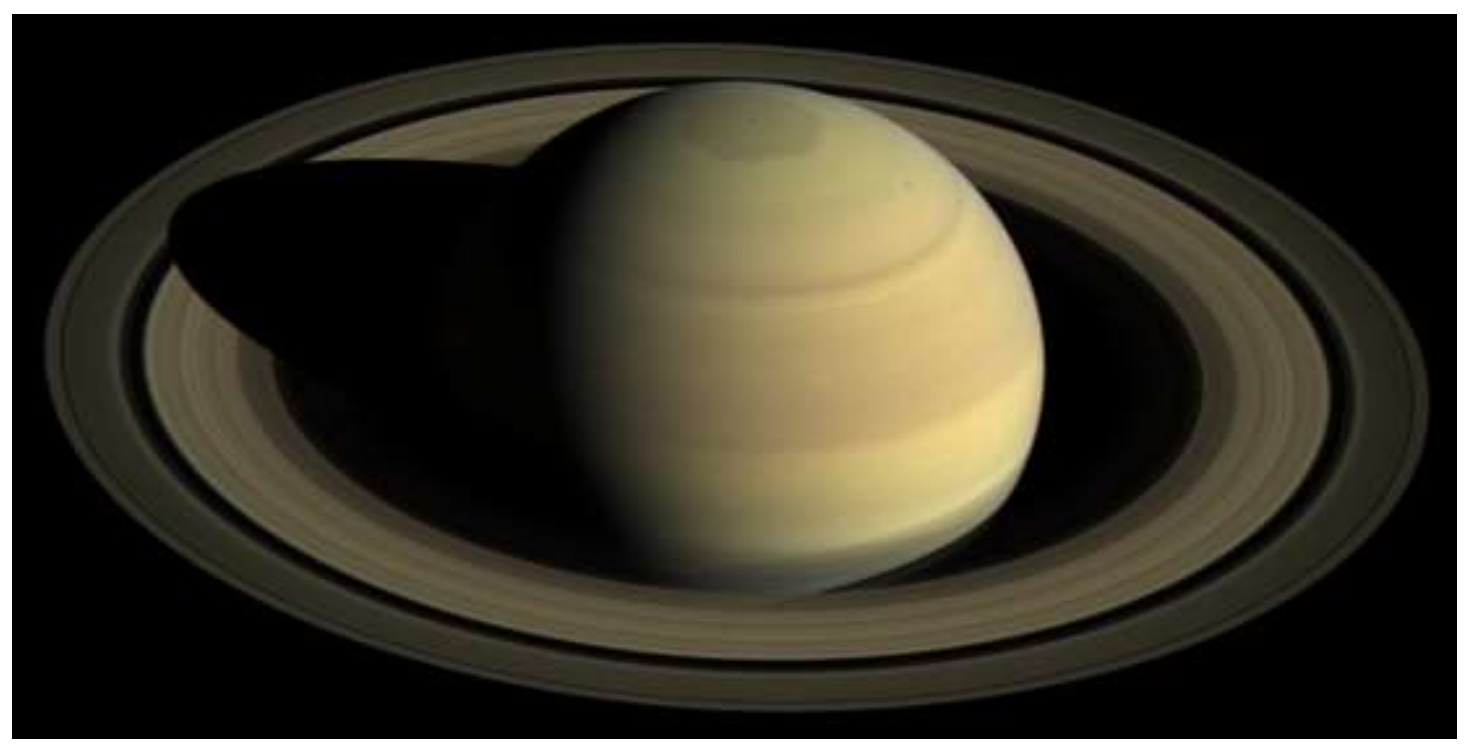

Figure 2. Saturn and its Rings.

Saturn is probably most known for its spectacular rings. While the other gas giants have rings, Saturn's are by far the largest with the highly visible A ring extending out to approximately 141,000 km from Saturn's center. Figure 3 illustrates the location and range of Saturn's rings, named alphabetically in order of discovery, as well as the location of some of Saturn's more prominent moons. 


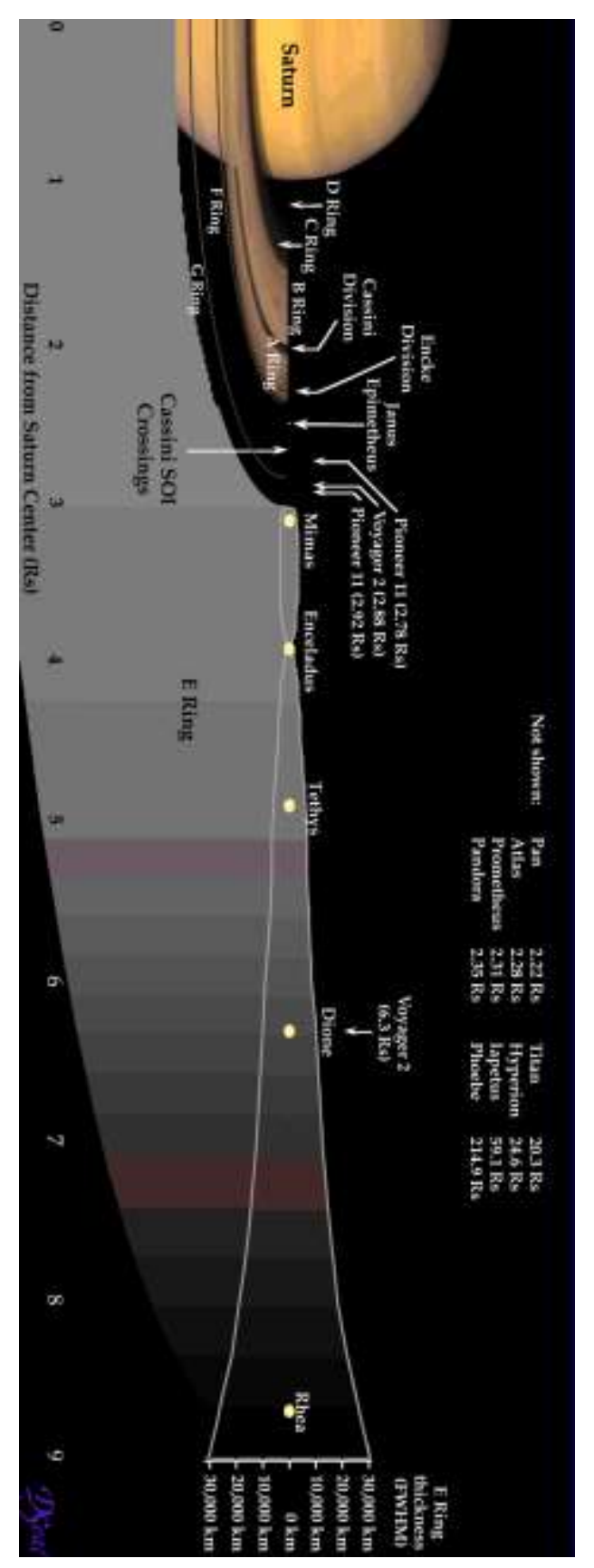

Figure 3. Diagram of Saturn's Rings and Important Moons.

\subsection{Cassini}

Before Cassini, only Pioneer 11, Voyager 1 and Voyager 2 had visited Saturn but these spacecraft only flew past and were not able to conduct extensive studies of the Saturn system [6]. Cassini became the first spacecraft to enter orbit around Saturn and continue the exploration of the outer gas giant. Before Cassini, only Jupiter had been extensively studied having been orbited by the Galileo probe [7]. Studying the gas 
giants helps constrain the origins and evolution of our solar system providing scientists with important information to be able to predict what will happen in the future. With the recent discovery of exoplanets, understanding the origins and evolution of planets within our own system helps determine the origin and evolution of body's in these extra-solar systems.

The Cassini spacecraft launched on October 15, 1997 from Cape Canaveral Airforce Station in Florida [8]. Cassini was lofted into space by a Titan IV rocket that at the time was the most powerful launch vehicle produced by the U.S. [8]. The Titan rocket provided Cassini with a C3 $18.1 \mathrm{~km}^{2} / \mathrm{s}^{2}$ [9]. At launch, the wet mass of Cassini was 5,712 kg. Only the two Phobos spacecraft developed by the Soviet Union were heavier, making Cassini one of the heaviest and most complex interplanetary spacecraft ever built [8]. Dry, Cassini's mass was $2125 \mathrm{~kg}$ [8] which does not include the $320 \mathrm{~kg}$ Huygens probe that Cassini carried to Saturn and released into Titans atmosphere. Fuel mass for Cassini was $3132 \mathrm{~kg}$.

Cassini inserted into Saturn Orbit on July $1^{\text {st }} 2004$ after an almost seven year journey and a 96 minute burn to change its velocity by almost $626 \mathrm{~m} / \mathrm{s}$ [10]. Cassini ended its four year primary mission on May $31^{\text {st }}$ 2008 [6]. Cassini followed a "ball of yarn" like trajectory on its tour through the Saturn system. During its tour, Cassini reached a maximum inclination in relation to Saturn's equator of $75.6^{\circ}$ and minimum inclination $0.3^{\circ}$ [11]. Cassini performed 45 close flybys of Titan that provided changes of inclination without burning fuel. Each of the close flybys also was used to perform various science investigations using Cassini's 12 instruments. The 45 flybys were useful to provide gravity assists to Cassini but Titan was also one of the primary scientific targets of the mission. Titan is the only moon in the solar system with a dense atmosphere and rocky core. Any planetary body with a solid surface and atmosphere is of interest because it helps scientists understand the formation of our home planet and how to better model our atmosphere on Earth. Titan's dense atmosphere also serves to block observations from Earth so only instruments in Titan's immediate vicinity can provide the data scientists desire. During tour, Cassini also performed three close flybys of Enceladus and one each of Dione, Rhea, Tethys, Hyperion, Phoebe, Mimas and Iapetus. 


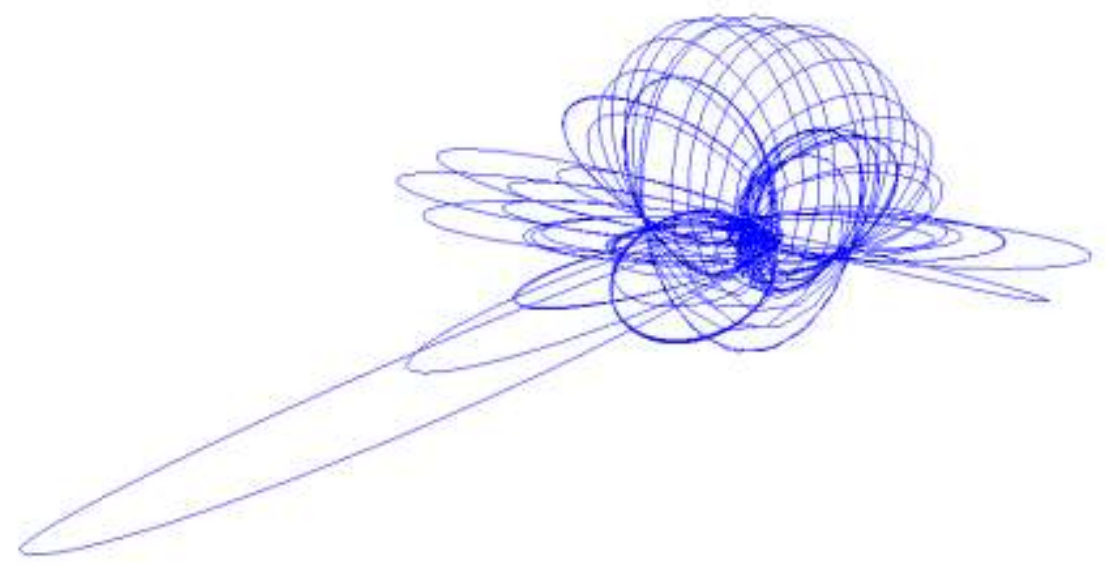

Figure 4. Cassini "Ball of Yarn" Trajectory.

Cassini was powered by three radioisotope thermal generators (RTGs) with each having a mass of $56.4 \mathrm{~kg}$ [12]. Cassini also used 82 radioisotope heater units (RHUs) to provide nearly $82 \mathrm{~W}$ of heat to keep Cassini warm in the depths of space [13]. Cassini was three axis stabilized using a combination of reaction wheels and thrusters to maintain pointing at all times during the mission. The reaction wheels, coupled with star trackers and gyroscopes, provided Cassini with a pointing accuracy of $2.0 \mathrm{mrad}$ [10]. Cassini had a $4 \mathrm{~m}$ parabolic high gain antenna which it used to downlink telemetry at a rate of $1 \mathrm{~Gb}$ per 12 hour track with a DSN $34 \mathrm{~m}$ high efficiency station an $4 \mathrm{~Gb}$ per 12 hour track with a $70 \mathrm{~m}$ station [14]. The Cassini telecommunication system operated at $13.8 \mathrm{GHz}$ and outputs $20 \mathrm{~W}$ of RF power to the antenna which has a gain of 47.2 dBi [14]. Two redundant Kaiser-Marquardt R4-J $490 \mathrm{~N}$ main engines are used to provide Cassini's anticipated $2040 \mathrm{~m} / \mathrm{s}$ of delta V [15]. Sixteen $1 \mathrm{~N}$ reaction control thrusters, two redundant sets of eight, were used to desaturate the reaction wheels and provide coarse attitude control [15].

Cassini was equipped with 12 scientific instruments; five remote sensing instruments, six fields and particles instruments and one radio science instrument. Four of the five remote sensing instruments composed the remote sensing pallet on the Cassini orbiter. Those four where the Imaging Science Subsystem (ISS) to capture images in the visible light spectrum, the Visual and Infrared Mapping Spectrometer (VIMS) to add additional spectral information to the images produced by the ISS, the Composite Infrared Spectrometer (CIRS) to measure thermal emmisions, and the Ultraviolet Imaging Spectrograph (UVIS) to measure ultraviolet energies. These instruments are passive remote sensing 
instruments in that they observe reflected energy from the target with the usual source of the energy being our sun. These instruments are also coaligned so they can observe the same target at once. The fifth instrument remote sensing instrument is the Cassini Radar which is an active remote sensing instrument in that it produces its own energy and measures the response from it.

The six fields and particles instruments are non-targeting in that they simply collect data on the immediate environment around the spacecraft and don't actively point to specific targets. These instruments are the Cassini Plasma Spectrometer (CAPS), which measures the plasma environment, the Cosmic Dust Analyzer (CDA), which determines size and density dust particles, the Ion and Neutral Mass Spectrometer (INMS), which determines elemental constituents of gases and atmospheres, the Magnetometer instrument (MAG), to study the magnetic field of Saturn, the Magnetospheric Imaging Instrument (MIMI), which constructs images of the interaction of the solar wind and Saturn's magnetosphere, and the Radio and Plasma Wave Science (RPWS), instrument to investigate plasma and radio wave emitted by Saturn.

The twelfth instrument is the Radio Science Instrument (RSI) which is built into Cassini's Communication system and performs radio science on the atmospheres of Saturn and Titan. The RSI has two primary components, one on the spacecraft and one back on Earth to receive and process the radio signals generated by the RSI. Table 1 summarizes the mass, power and use of each of Cassini's instruments. Figure 5 and Figure 6 show detailed views of the Cassini spacecraft.

Table 1. Cassini Instrument Overview.

\begin{tabular}{|l|l|l|l|}
\hline Instrument & Mass $(\mathbf{k g}$ & Power $\mathbf{( W )}$ & Use \\
\hline ISS & 57.83 & 55.9 & Visual imaging \\
\hline VIMS & 37.14 & 27.2 & Visual spectroscopy \\
\hline CIRS & 39.24 & 32.9 & IR spectroscopy \\
\hline UVIS & 14.46 & 11.8 & UV Spectroscopy \\
\hline Radar & 41.43 & 108.4 & Imaging and altimetry \\
\hline CAPS & 12.50 & 14.5 & Plasma science \\
\hline CDA & 16.36 & 18.4 & Dust analysis \\
\hline INMS & 9.25 & 27.7 & Mass spectroscopy \\
\hline Mag & 3.0 & 3.1 & Magnetic fields \\
\hline MIMI & 16 & 14.0 & Solar wind interaction \\
\hline RPWS & 6.80 & 7.0 & Radio and plasma wave \\
\hline Radio & 14.38 & 80.7 & Radio science \\
\hline Total & 268.4 & 401.6 & \\
\hline
\end{tabular}




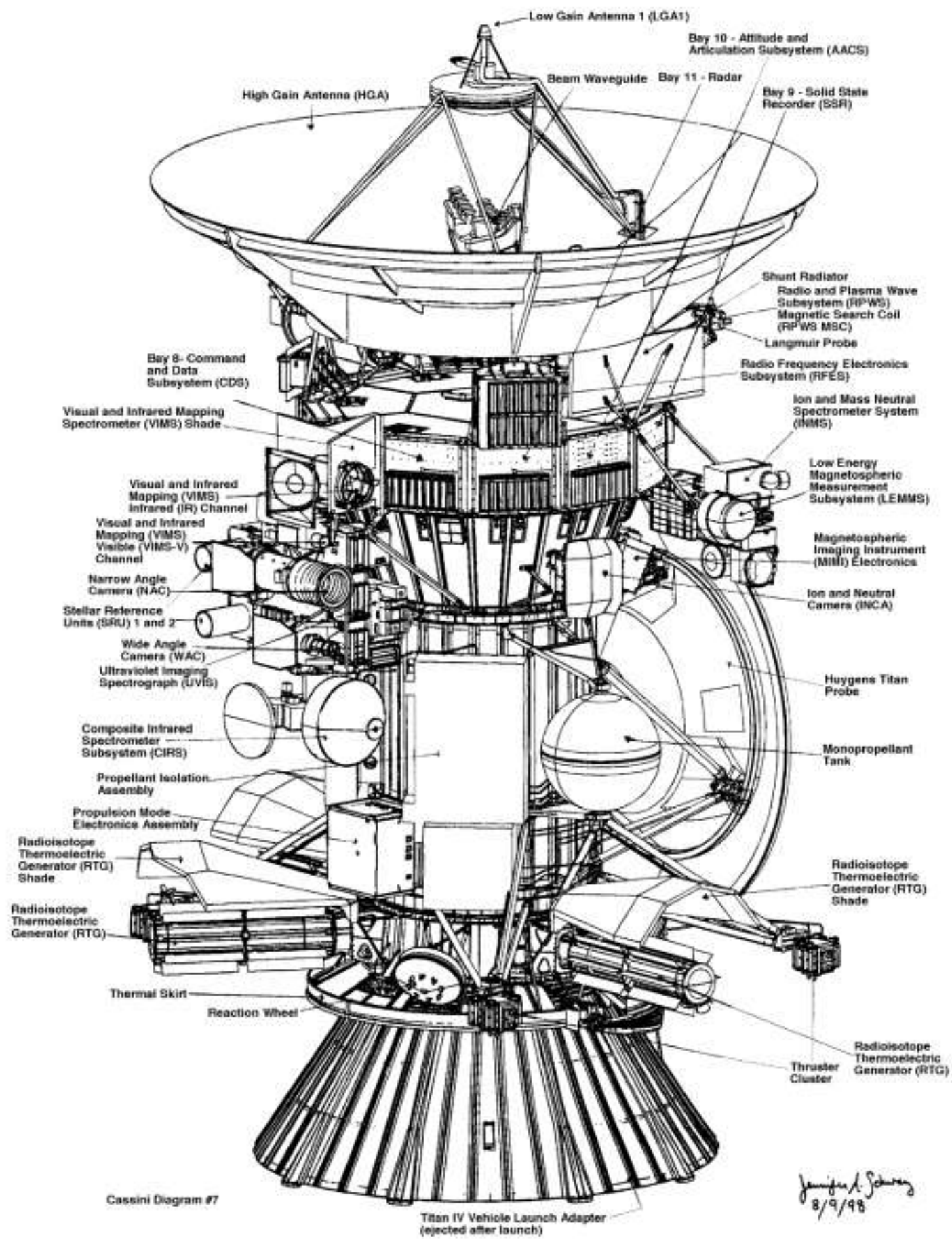

Figure 5. Cassini Diagram: Remote Sensing Side. 


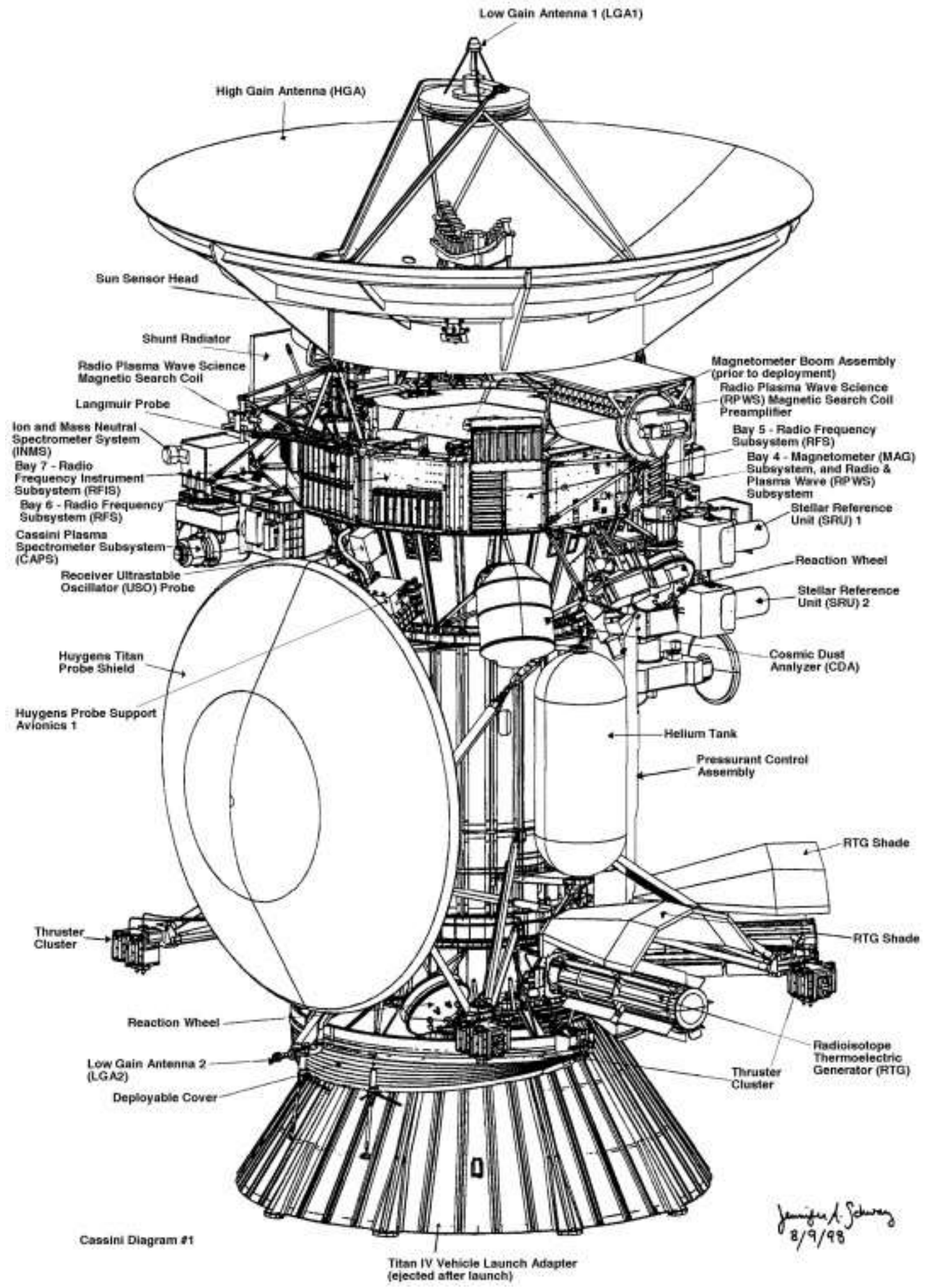

Figure 6. Cassini Diagram: Huygens Probe Side. 


\subsubsection{Imaging Science Subsystem (ISS)}

The ISS consists of a narrow and a wide-angle camera. The two cameras work together to image the Saturn system and are used by Cassini for optical navigation. The Narrow Angle Camera (NAC) is a Ritchey-Chretien reflective telescope and is used for high resolution images of specific targets. The NAC gets its magnification from its long focal length of $2 \mathrm{~m} \mathrm{[16].} \mathrm{At} \mathrm{the} \mathrm{time} \mathrm{Cassini} \mathrm{was} \mathrm{launched,} \mathrm{the} \mathrm{NAC}$ had the longest focal length optics of any interplanetary probe [16]. The Wide Angle Camera (WAC) in contrast, has a much shorter focal length and uses refractive lenses similar to standard camera optics used by photographers. The shorter focal length gives the WAC ten times the field of view of the NAC [16] and is used to provide context to the images produced by the NAC.

Both cameras are sensitive to a wide range of wavelength from near ultraviolet to near infrared with the NAC having a wider range being more sensitive to ultraviolet. The cameras have multiple filters that can be used to take images at specific wavelengths as opposed to panchromatic images over the whole spectral range. These filters allow for different colors to be imaged which not only produces standard color pictures that we see but also gives scientific insight to the structure and composition of the areas being imaged. The light that the human retina is sensitive to, otherwise known as visible light, ranges from approximately 390 to $700 \mathrm{~nm}$. The NAC and WAC however are sensitive to wavelength from 200 to $1100 \mathrm{~nm}$ and 380 to $1100 \mathrm{~nm}$ respectively [16]. The wide spectral range of wavelengths combined with the filters allow the cameras to "see” things humans can’t.

Each filter is specially chosen for different science investigations. For example, the cameras have filters at $938 \mathrm{~nm}$ to view the Titan surface [16]. Titan's atmosphere is such that there is a very narrow window through which light can pass unscattered by the atmospheric constituents and so this filter allows the ISS to look through this window. Because the WAC has a smaller spectral range, it has only 18 filters as opposed to the NAC's 24 filters [16]. The WAC has a smaller range because its uses refractive lenses which do not permit UV light to pass through. The NAC uses reflective mirrors which are reflective to a wider range of wavelengths and is only limited by the sensitivity of the sensor receiving the light.

Both cameras have the same sensor located at their focal point. The sensors are 1024 x 1024 pixel area sensor [16]s. Area sensors are used as opposed to line scanners which have a single line of pixels that 
are then "pushed" along by the motion of the spacecraft. Area sensors allow the ISS to stare at a target for extended periods of time and produce a full image. ISS's sensors, built in the 1990's, where one megapixel each. Today, even phone cameras can have sensors in the tens of megapixel range.

Figure 7 shows the NAC and WAC mounted together to comprise the ISS. Table 2 gives the specifications of the ISS instrument.

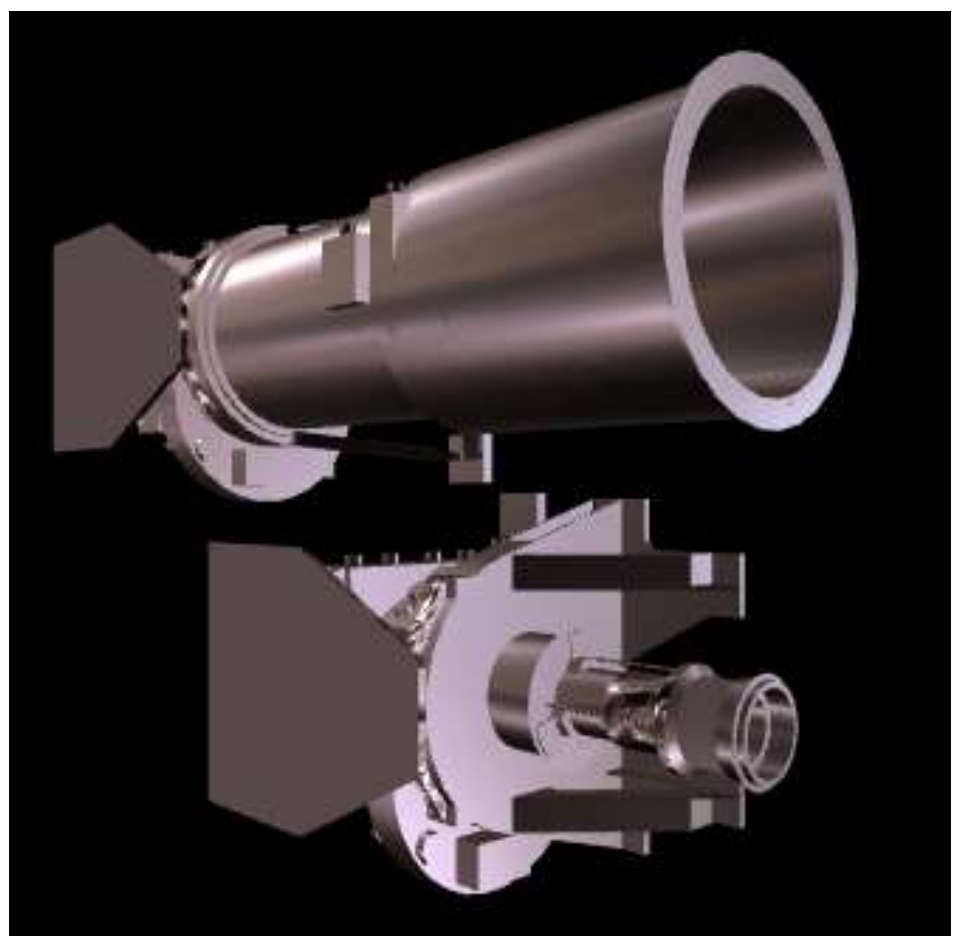

Figure 7. Cassini ISS. The NAC is on top with the WAC on bottom. 
Table 2. ISS Specifications [16].

\begin{tabular}{|c|c|c|}
\hline & NAC & WAC \\
\hline Mass & $30.5 \mathrm{~kg}$ & 26.4 \\
\hline Dimensions & $95 \times 40 \times 33 \mathrm{~cm}$ & $55 \times 35 \times 33 \mathrm{~cm}$ \\
\hline Power & $26.2 \mathrm{~W}$ & $19.4 \mathrm{~W}$ \\
\hline Sensor & $\mathrm{CCD}$ & $\mathrm{CCD}$ \\
\hline Pixel Size & $12 \mu \mathrm{m}$ & $12 \mu \mathrm{m}$ \\
\hline Format & 1024 x 1024 pixels & $1024 \times 1024$ pixels \\
\hline Exposure times & $\begin{array}{c}64 \text { commandable settings, } 5 \mathrm{~ms}- \\
1200 \mathrm{~s}\end{array}$ & $\begin{array}{c}64 \text { commandable settings, } 5 \mathrm{~ms}- \\
1200 \mathrm{~s}\end{array}$ \\
\hline Framing Time & $11 \mathrm{~s}$ & $11 \mathrm{~s}$ \\
\hline Pixel Full Well & $120,000 \mathrm{e}-$ & $120,000 \mathrm{e}-$ \\
\hline Read Noise & $12 \mathrm{e}-$ & $12 \mathrm{e}-$ \\
\hline F\# & 10.5 & 3.5 \\
\hline Focal Length & $2002.7 \mathrm{~mm}$ & $200.77 \mathrm{~mm}$ \\
\hline IFOV & $5.9907 \mu \mathrm{rad}$ & $59.749 \mu \mathrm{rad}$ \\
\hline FOV & $6.134 \mathrm{mrad}$ & $61.18 \mathrm{mrad}$ \\
\hline PSF & 1.3 & 1.8 \\
\hline Spectral Range & $200-1050 \mathrm{~nm}$ & $380-1050 \mathrm{~nm}$ \\
\hline Number of Filters & 24 & 18 \\
\hline & \multicolumn{2}{|c|}{ Total } \\
\hline Mass & \multicolumn{2}{|c|}{$57.83 \mathrm{~kg}$} \\
\hline Peak Power & \multicolumn{2}{|c|}{$55.9 \mathrm{~W}$} \\
\hline Peak Data Rate & \multicolumn{2}{|c|}{$365.6 \mathrm{kbps}$} \\
\hline
\end{tabular}

The overarching goals of the ISS are to develop an understanding of the origin and evolution of the Saturnian system to gain a better understanding of the four outer gas planets in general. Specific attention was given to comparing Saturn to Jupiter, which was the most studied gas planet at that time, in order to compare the two planets evolution. All four gas planets were formed from the same protoplanetary nebula and so understanding the formation of Saturn provides clues to the formation of the all four planets and better refine models for the formation of the solar system.

The ISS images the surface of satellites to create high resolution geodetic maps and take multiple geometry images to characterize the surface structure, geologic resurfacing and crater size and distribution. Surface characterization helps determine geologic processes and surface age. Images of the satellites will determine albedo of the objects as well as their size, mass and shape which are used to formulate the history and origins of the Saturn system and determine unique qualities of Saturn in comparison to the other gas planets. 
The ISS tracks clouds in the atmosphere to perform wind speed measurements and understand meteorological processes in general. Because Saturn is farther away from the sun and gets less energy, its atmosphere is colder and so the cloud deck is lower and obscured by aerosols making for poor imaging from Earth based telescopes and a lack of understanding of the atmosphere.

Characterization of the ring structure at high spatial resolution and broad spectral range to determine age, mass, and dynamics is performed by the ISS. Images taken are compared to those taken by Voyager 1 to characterize temporal changes in the rings and update models created from the Voyager data

The ISS has several filters to assist in viewing the surface of Titan. Titan's atmosphere is composed mostly of nitrogen but contains a small amount of methane. This methane is highly absorbent to light and plays a large role in obscuring Titan's surface from remote sensing. However, there are several spectral windows through which light can pass and the filters enable the ISS to look through them at Titan's surface

ISS also has polarizing filters which are important for Titan aerosol studies as well as surface viewing. Imaging the atmosphere during occultations of the sun with the polarizing filters give clues to the nature of the aerosol constituents. The same aerosols scatter the light coming from the surface of Titan making imaging terrestrial features difficult. The polarizing filters of the ISS cut down this scattered light similar to how polarized sunglasses sharpen scenes on Earth.

\subsubsection{Visible and Infrared Mapping Spectrometer (VIMS)}

The Cassini VIMS is a hyperspectral mapping spectrometer covering the visible and short wave IR spectrum. VIMS is composed of two instruments, the VIMS-VIS which is covers the visible spectrum and the VIMS-IR which covers the IR spectrum. The two instruments overlap in their spectral range to continuously cover wavelengths from $300-5100 \mathrm{~nm}$ [17]. 


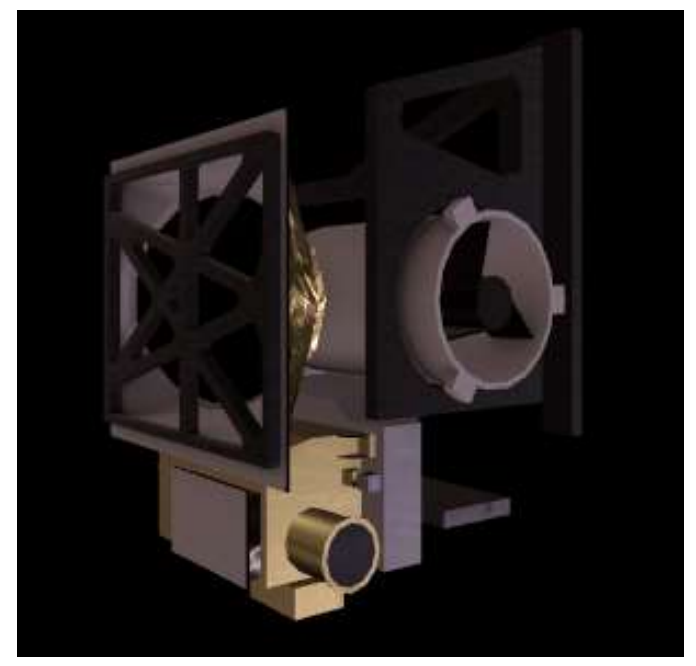

Figure 8. Cassini VIMS Instrument with VIMS-VIS bottom left and VIMS-IR top right.

Mapping spectrometers create a three-dimensional image product with the third image being spectral information. For each pixel in a normal two-dimensional image, a mapping spectrometer also records the spectral intensity at different bands which forms the third dimension of the product. An image "cube" can be visualized by the example shown in Figure 9. The third dimension of spectral information enables the identification of the object or material in the area of the pixel. For VIMS and Cassini, different gases in the atmospheres of Saturn and Titan and different compounds in the surface of the icy satellites and rings have different spectral signatures that can be identified and distinguished by VIMS.

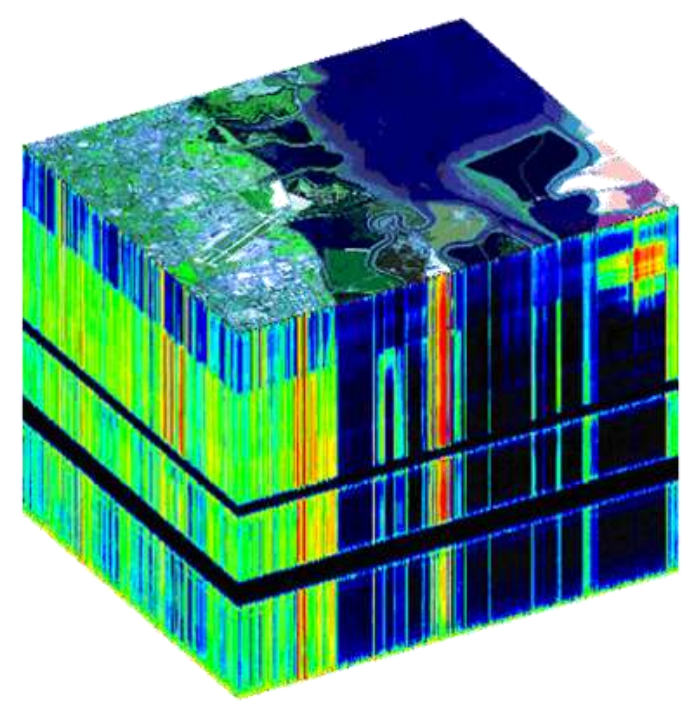

Figure 9. Spectrometer 3D image "cube". 
VIMS-VIS has 96 channels and is covers $300-1000 \mathrm{~nm}$ [17]. The 96 bands are each $7.3 \mathrm{~nm}$ wide and evenly divided along its $700 \mathrm{~nm}$ range [17]. The optics are a Schafer design telescope which incorporates and inverted Burch telescope with an Offner relay. VIMS-VIS is a grating spectrometer which utilizes a grated plane to divide the incoming light into its spectral constituents much like a prism. The light falls onto a 256 x 480 spatial x spectral pixel CCD [17]. Because the 480 pixel direction is purely spectral, the result is one dimensional linear spatial array. In order to create a two dimensional image, the primary mirror of the Burch telescope scans in the across track dimension in a whisk broom architecture to create the image. VIMS-VIS does a $3 \times 5$ pixel summation resulting in 83 spatial pixels and 96 spectral pixels to create the 96 bands [17]. The spatial pixels are further reduced to 64 to match the field of view of VIMS-IR [17].

VIMS-IR has 256 spectral channels spanning 850 - $5100 \mathrm{~nm}$ with each channel $16.6 \mathrm{~nm}$ wide [17]. The IR fore optics is a Ritchy-Cretien telescope with a $23 \mathrm{~cm}$ aperture operating at F3.5 [17] which is coupled to a Dahl-Kirkham collimator. VIMS-IR is also a grating spectrometer splitting the light onto a 1x256 linear InSb array [17]. Because the array is linear, it is purely spectral and has no spatial component. To create a spatial image, the primary mirror can be scanned in two dimensions to create the 2-D image. The VIMS-IR focal plane is cooled to 55-60 K using an active cold finger [17].

Table 3. VIMS Specifications [17].

\begin{tabular}{|c|c|c|}
\hline & VIMS-VIS & VIMS-IR \\
\hline Sensor & $\mathrm{CCD}$ & $\mathrm{CCD}$ \\
\hline Pixel Size & $24 \mu \mathrm{m}$ & $200 \times 103 \mu \mathrm{m}$ \\
\hline Pixel Format & $64 \times 96$ & $1 \times 256$ \\
\hline F\# & 3.2 & 3.5 \\
\hline Aperture & & $23 \mathrm{~cm}$ \\
\hline IFOV & $0.5 \mathrm{mrad}$ & $0.5 \mathrm{mrad}$ \\
\hline FOV & $32 \mathrm{mrad}$ & $32 \mathrm{mrad}$ \\
\hline PSF & 1.3 & 1.8 \\
\hline Spectral Range & $300-1000 \mathrm{~nm}$ & $850-5100 \mathrm{~nm}$ \\
\hline Number of Bands & 96 & 256 \\
\hline Spectral Resolution & $7.3 \mathrm{~nm}$ & $16.6 \mathrm{~nm}$ \\
\hline & \multicolumn{2}{|c|}{ Total } \\
\hline Mass & \multicolumn{2}{|c|}{$37.14 \mathrm{~kg}$} \\
\hline Peak Power & \multicolumn{2}{|c|}{$27.2 \mathrm{~W}$} \\
\hline Peak Data Rate & \multicolumn{2}{|c|}{$182.8 \mathrm{kbps}$} \\
\hline
\end{tabular}


The VIMS primary goals are to investigate the chemical and dynamical processes of Saturn and Titan to further our knowledge of the formation and evolution of the Saturnian and solar system. The threedimensional nature of the data produced by VIMS will provide insight to the thermal, aerosol, and chemical structures of the atmospheres of Saturn and Titan as well as the physical makeup of the other Saturnian satellites and the rings. Determining the structure of these atmospheres and the ratios of gaseous elements and isotopes will allow constraints to be placed on the formation history of the Saturnian system and by extension the other icy giants and even the solar system as a whole.

The VIMS instrument is coaligned with the ISS allowing the two instruments to work together. The ISS provides high spatial resolution and context with VIMS providing the spectral makeup and constituent classification of the frame in view. VIMS is also coaligned with CIRS and UVIS. CIRS extends the spectral range tremendously into the long range IR and UVIS looks at the extreme UV spectrum the three spectrometer complement each other and provide a complete picture of the target.

\subsubsection{Composite Infrared Spectrometer (CIRS)}

The CIRS instrument consists of two interferometer spectrometers that share the same telescope optics to cover the spectral range of $7000-1,000,000 \mathrm{~nm}$ [18]. One interferometer covers the far IR range of 17000$1,000,000 \mathrm{~nm}$ and the other covering the mid IR range of $7000-17000 \mathrm{~nm}$ [18]. The telescope has an aperture of $50.8 \mathrm{~cm} \mathrm{[18].}$

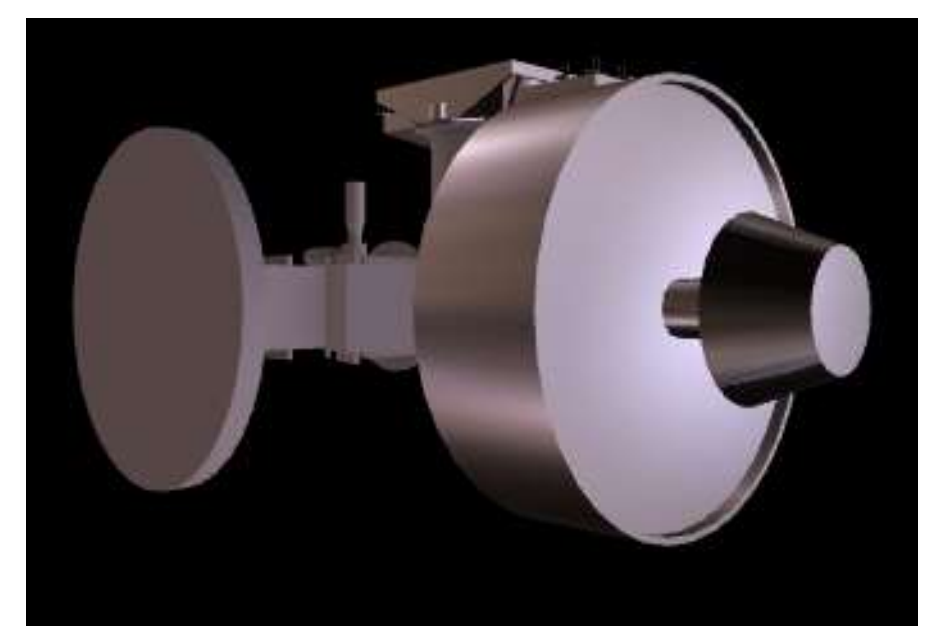

Figure 10. CIRS Instrument. 
Both spectrometers are Michelson Fourier Transform spectrometers. Instead of splitting the incoming light with a prism or grating like VIMS, Fourier spectrometers split the light into two beam paths of differing lengths then recombine the beams in such a way that, because of the different path lengths, the beams of light destructively interfere creating a filter for selected wavelengths. A photodetector then measures the intensity of the remaining light to get a sample. The process repeats with changing path lengths to create a complete data set. Onboard computer processing then applies a Fourier transform algorithm to the data set to determine the spectral signature of the target. A common interferometer arrangement is shown in Figure 11. The advantages of using a Fourier Transform Spectrometer are that they are capable of measuring much greater spectral ranges at finer spectral resolutions allowing for the detection of narrow atmospheric absorption window across a broad spectral range.

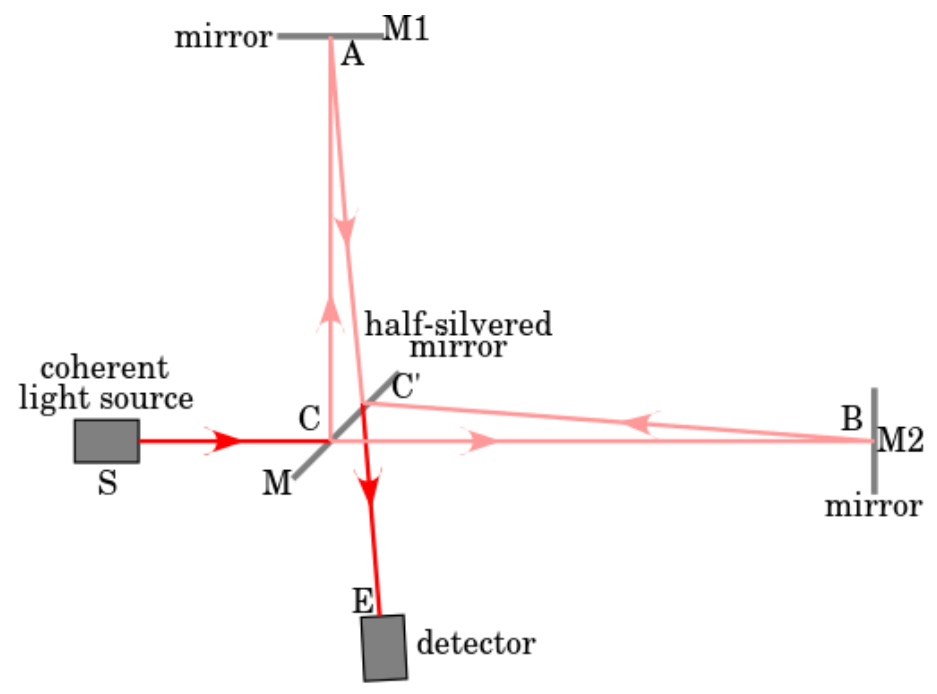

Figure 11. Michelson Interferometer.

Far IR interferometer, covering 17,000 - 1,000,000 nm, has a single thermopile detector pixel. The thermopile pixel has the best performance in the far IR wavelengths and is operated at $170 \mathrm{~K}$ [18]. the thermopile detector was chosen for its high sensitivity, low response time and low noise.

Mid IR interferometer has two focal planes, one for the wavelength range of $7000-9000 \mathrm{~nm}$ and the other for 9000 - $17000 \mathrm{~nm}$ [18]. Each focal plane has a linear array of ten $\mathrm{HgCdTe}$ detector pixels and each pixel has a field of view of $0.273 \operatorname{mrad}[18]$. 
Table 4. CIRS Specifications [18].

\begin{tabular}{|l|l|l|l|}
\hline & Far IR & Mid IR FP1 & Mid IR FP2 \\
\hline Spectral Range & $17-1000 \mu \mathrm{m}$ & $9-17 \mu \mathrm{m}$ & $7-9 \mu \mathrm{m}$ \\
\hline Spectral Resolution & $0.5-15.5 \mathrm{~cm}^{-1}$ & $0.5-15.5 \mathrm{~cm}^{-1}$ & $0.5-15.5 \mathrm{~cm}^{-1}$ \\
\hline Pixel Format & $1 \times 1$ & $1 \times 10$ & $1 \times 10$ \\
\hline IFOV & $3.9 \mathrm{mrad}$ & $0.273 \mathrm{mrad}$ \\
\hline Detector Temp & $170 \mathrm{~K}$ & $0.273 \mathrm{mrad}$ & $75-90 \mathrm{~K}$ \\
\hline & & $75-90 \mathrm{~K}$ & \\
\hline Mass & & Total & \\
\hline Power & & $39.24 \mathrm{~kg}$ \\
\hline Data Rate & & $26.37 \mathrm{~W}$ \\
\hline
\end{tabular}

The thermal IR wavelengths are ideal to observe the Saturn system due to its cold radiating temperatures of 55-200 K [18]. CIRS will observe Saturn and Titan to measure temperatures in the atmospheres to better understand what drives their weather patterns. CIRS will also perform many limb view observations where instead of pointing towards nadir, it points at the very edge of the planet or satellite to focus on the region between the "surface" and outer reaches of the atmosphere. Figure 12 illustrates limb vs nadir viewing. Limb viewing will allow the CIRS instrument to profile the atmospheric temperatures at different altitudes simultaneously to better model the atmosphere and understand thermal drivers in atmospheric winds. Limb viewing does not rely on an occultation event allowing CIRS to observe the atmosphere at any latitude or longitude enabled by its orbit. CIRS will also be able to map surface temperature of Saturn's satellites to help identify compositional features.

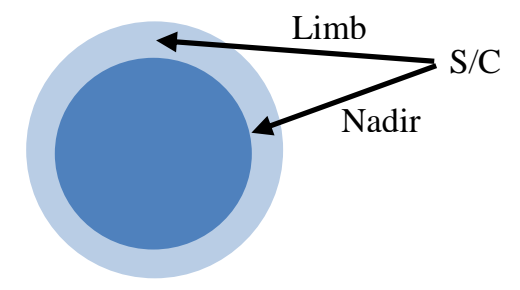

Figure 12. Limb vs Nadir Observations.

CIRS is an excellent complement to VIMS as CIRS starts its spectral range where VIMS stops. Where VIMS is designed to identify aerosols and other atmospheric constituents, CIRS will measure temperature 
profiles and determine abundances of elements in Saturn and Titan's atmospheres. Both instruments will help determine the fundamental differences in structure and composition between Jupiter and Saturn.

\subsubsection{Ultraviolet Imaging Spectrograph (UVIS)}

UVIS is a hyperspectral imager operating in the far and extreme UV wavelengths from $56-191 \mathrm{~nm}$. Wavelengths from $56-118 \mathrm{~nm}$ are covered by one telescope and detector plane while the wavelengths from $111-191 \mathrm{~nm}$ are covered by a separate detector and telescope pair. The two spectrographic channels are grating spectrometers like VIMS with the light falling on a 1024 spectral x 64 spatial pixel detector.

UVIS also contains additional channels for high speed photometry and a Hydrogen Deuterium Absorption Cell (HDAC). The High Speed Photometer (HSP) has an integration time of just $2.0 \mathrm{~ms}$ to capture fleeting stellar occultations of Saturn's rings. The HSP is sensitive to wavelengths between 115 and $190 \mathrm{~nm}$ but is not a spectrometer in that it only has one detector pixel that is sensitive to the entire spectral range. This allows the HSP to have such a low integration time but has the drawback of only being able to determine if a target is emitting UV light or not. The detector for the HSP is a CsI photocathode that has a low sensitivity to solar light to allow it to not pick up light emitted from the star during an occultation.

The HDAC is used to measure the relative signal strengths of the lyman- $\alpha$ emission lines of hydrogen and its isotope deuterium. Using the relative signal strengths, a direct measurement of the hydrogen to deuterium ratio can be made. Deuterium is a naturally occurring isotope of hydrogen and determining the ratio between the two helps determine when and where the body in question formed and whether it was supplemented by comets.

The UVIS instrument is coaligned with the other remote sensing instruments on the remote sensing platform of Cassini. The complete UVIS instrument is shown in Figure 13. Its specifications are listed in Table 5. 


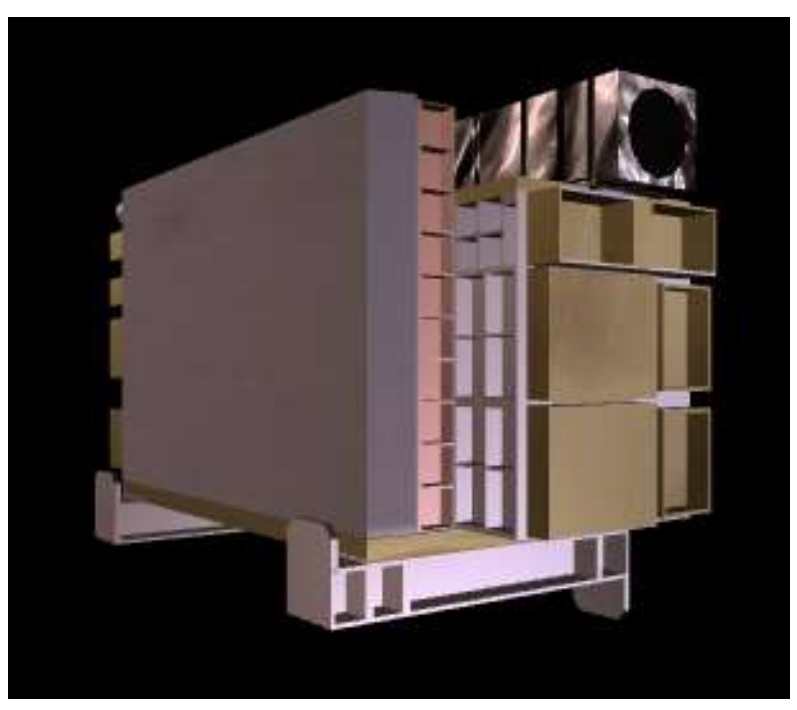

Figure 13. UVIS Instrument.

Table 5. UVIS Specifications [19].

\begin{tabular}{|l|l|l|l|}
\hline & Far UV & Extreme UV & HSP \\
\hline Spectral Range & $111.5-191.2 \mathrm{~nm}$ & $56.3-118.2 \mathrm{~nm}$ & $115-190 \mathrm{~nm}$ \\
\hline Spectral Resolutions & $0.275,0.48,2.49 \mathrm{~nm}$ & $0.275,0.48,1.94 \mathrm{~nm}$ & N/A \\
\hline \# Bands & 1024 & 1024 & 1 \\
\hline FOVs & $0.75,1.5,8.0 \times 60 \mathrm{mrad}$ & $1,2,8, \times 59 \mathrm{mrad}$ & $6.0 \mathrm{mrad}$ \\
\hline Pixel Format & $1024 \times 64$ & $1024 \times 64$ & $1 \times 1$ \\
\hline Focal Length & $100 \mathrm{~mm}$ & $100 \mathrm{~mm}$ & $200 \mathrm{~mm}$ \\
\hline Aperture & $20 \times 20 \mathrm{~mm}$ & $20 \times 20 \mathrm{~mm}$ & $135 \times 30 \mathrm{~mm}$ \\
\hline & & \multicolumn{2}{|c|}{ Total } \\
\hline Mass & & $15.6 \mathrm{~kg}$ & \\
\hline Power & & $8.0 \mathrm{~W}$ & \\
\hline Data Rate & & $32.1 \mathrm{kbps}$ \\
\hline
\end{tabular}

UVIS is used for a host of scientific observations but primarily for atmospheric investigations and compositional determination of the rings. For Saturn and Titan, UVIS will map the distribution of UV absorbing aerosols. UVIS will map the altitude and latitude distribution of hydrogen and hydrocarbon molecules. UVIS will also investigate and observe the aurorae of Saturn to determine the interaction of its atmosphere and ionosphere. Using solar and stellar occultations, UVIS will detect whether sub-micron dust is present in the rings. Additional information on ring structure and variability as well as albedo distribution will be determined. 


\subsubsection{Radar}

The Radar instrument is designed primarily to investigate the surface of Titan but is also used for other Saturn satellites and Saturn itself. The instrument has four operational modes; synthetic aperture radar (SAR) imaging, altimetry, scatterometry, and radiometry. The Radar will investigate Titan in all four modes but only the scatterometry and radiometry modes will be used on other targets. The Radar instrument utilizes Cassini’s 4 m high gain antenna and operates at $13.8 \mathrm{GHz}$ [20].

SAR is used to produce radar images of a target. The Radar in SAR imaging mode differs from the remote sensing instruments in that it is an active imager. In active remote sensing, the instrument is both the signal detector and source. The Radar instrument sends a high-power radio frequency pulse, in this case at 13.8 $\mathrm{GHz}$, which reflects off the target and received by the detector in the instrument. Differing return times for the signal determine distance between the spacecraft and the target and multiple successive pulses help build a picture of the target. Synthetic aperture radar take the principle a step further by measuring the return signal at multiple locations to effectively enlarge the antenna size to produce a better image. SAR has the benefit of being its own signal source so it can image the target regardless of solar lighting conditions. The reason it is used for Titan is that its signal, being a radio frequency, can penetrate Titan's thick atmosphere and cloud layer to observe the surface which is something the other remote sensing instruments can't do. Due to the limited number of Titan flybys during the Primary mission, only $25 \%$ of Titans surface is expected to be imaged with SAR [20].

The altimeter operates similarly to the SAR mode in that it actively sends out pulses but instead of producing an image the altimeter produces detailed altitude information within the swath width and orbit track. Successive overlapping altimetry passes allow the Radar to map the altitude variations of Titan and a topographical map can be produced. The scatterometer mode effectively operates as a coarse altimeter. The scatterometer measures the surface back scatter of the radar signal and is often used for wind speed and ocean surface roughness measurements but will also determine back scatter characteristics of Titan.

The radiometer mode, unlike the others is not active, and is instead used to gather information on the radiofrequency emissions of the target itself. The radiometer can be used in conjunction with the other 
modes in between their pulses. The radiometer will measure the radiofrequency brightness temperature of the target.

During a typical flyby of Titan, all four modes will be used starting with radiometry 5 hours out and ending at closest approach with high resolution SAR imaging. After closest approach the order of observations will be reversed ending 5 hours past for a total Radar observation of 10 hours. The first five hours of observations are detailed in Figure 14. Figure 15 shows an image of the Radar instrument itself.

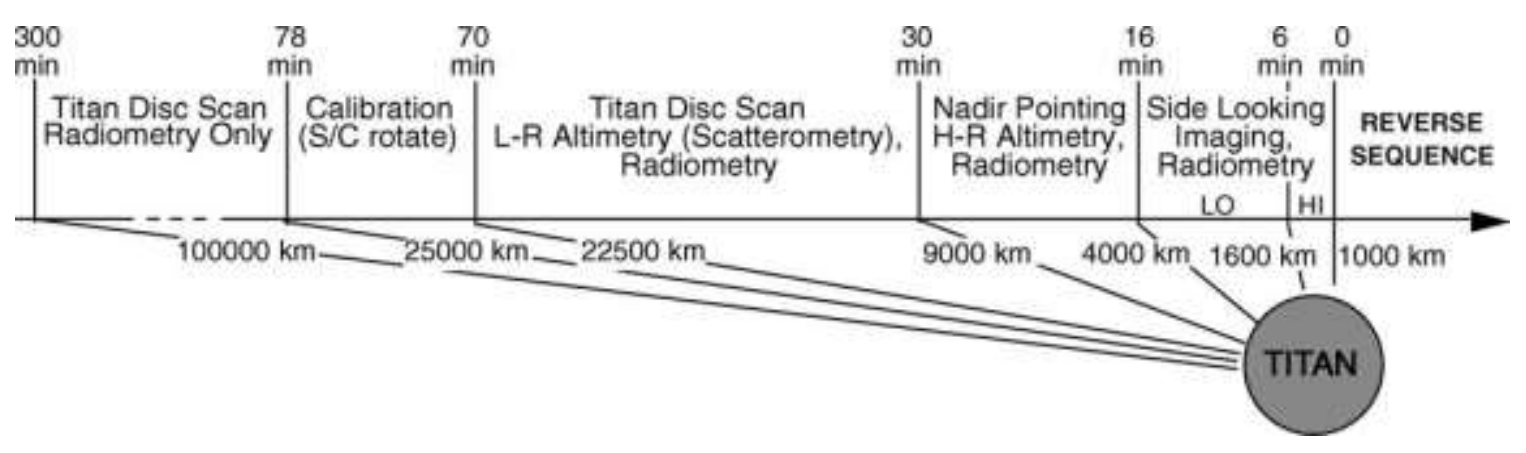

Figure 14. Radar Observations During a Titan Flyby [20].

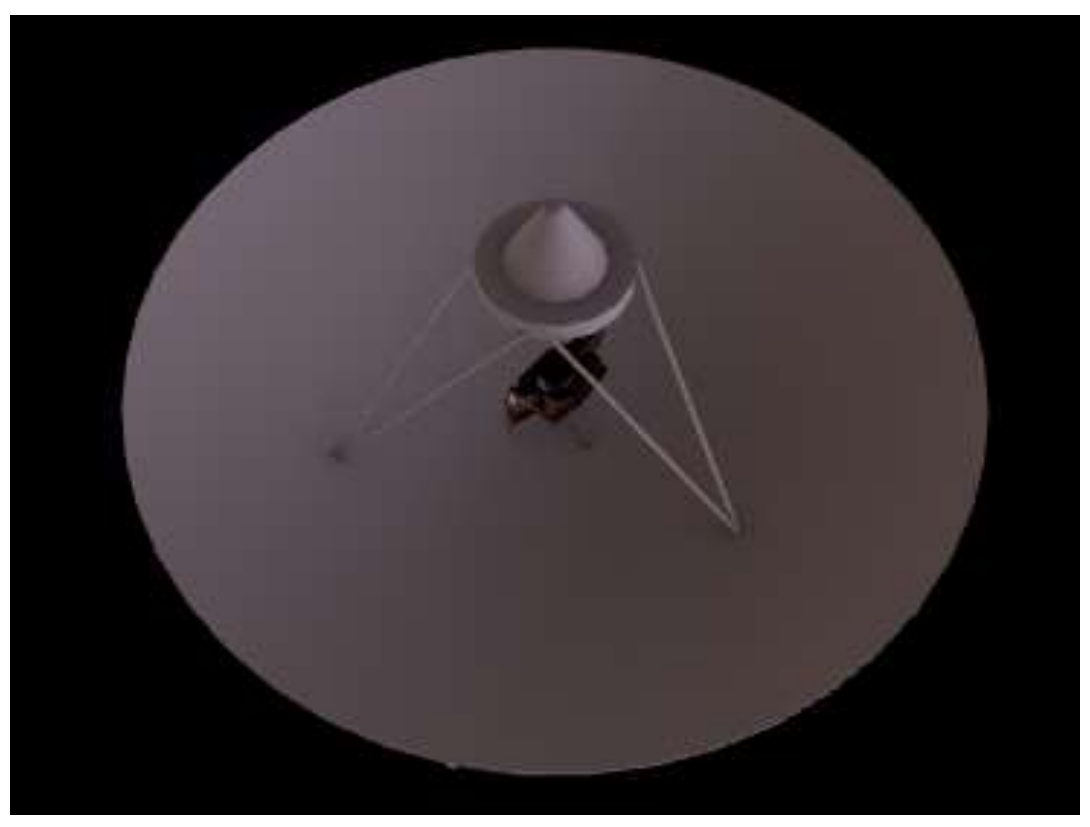

Figure 15. Radar instrument Antenna. 
Table 6. Radar Specifications [20].

\begin{tabular}{|c|c|c|c|c|c|c|}
\hline Antenna Diameter & \multicolumn{6}{|c|}{$4 \mathrm{~m}$} \\
\hline Frequency & \multicolumn{6}{|c|}{$13.8 \mathrm{GHz}$} \\
\hline & SAR High & SAR Low & Alt. & \multicolumn{2}{|l|}{ Scat. } & Radmtr. \\
\hline $\begin{array}{l}\text { Altitude of Operation } \\
(\mathrm{km})\end{array}$ & $1000-1600$ & $1600-4000$ & $4000-9000$ & \multicolumn{2}{|c|}{$9000-22500$} & $1000-100000$ \\
\hline Incidence Angle $\left(^{0}\right)$ & $21-30$ & $15-28$ & 0 & \multicolumn{2}{|l|}{$0-30$} & $0-8$ \\
\hline Azimuth Res (km) & $0.35-0.41$ & $0.41-0.72$ & $24-27$ & \multicolumn{2}{|l|}{$55-140$} & $6-600$ \\
\hline Range Res (km) & $0.48-0.64$ & $0.48-2.70$ & $24-27$ & \multicolumn{2}{|l|}{$55-140$} & $6-600$ \\
\hline Vertical Res (km) & N/A & N/A & 60 & \multicolumn{2}{|l|}{ N/A } & N/A \\
\hline & \multicolumn{2}{|c|}{ Beams 1 and 5} & \multicolumn{2}{|c|}{ Beams 2 and 4} & \multicolumn{2}{|c|}{ Beam 3} \\
\hline Peak Gain & \multicolumn{2}{|l|}{$42.0 \mathrm{~dB}$} & \multicolumn{2}{|l|}{$42.1 \mathrm{~dB}$} & \multicolumn{2}{|c|}{$49.1 \mathrm{~dB}$} \\
\hline Azimuth Beamwidth & \multicolumn{2}{|l|}{$0.35^{\circ}$} & \multicolumn{2}{|l|}{$0.35^{\circ}$} & \multicolumn{2}{|c|}{$0.35^{\circ}$} \\
\hline Cross-track Beamwidth & \multicolumn{2}{|l|}{$1.35^{\circ}$} & \multicolumn{2}{|l|}{$1.35^{\circ}$} & \multicolumn{2}{|c|}{$1.35^{\circ}$} \\
\hline Mass & \multicolumn{6}{|c|}{$43.3 \mathrm{~kg}$} \\
\hline Power & \multicolumn{6}{|c|}{$86 \mathrm{~W}$} \\
\hline Data Rate & \multicolumn{6}{|c|}{1 Gbit/ Titan Flyby } \\
\hline
\end{tabular}

The priority science investigations for the Radar instrument are the determination of the of the physical state, topography and composition of Titan's surface and a global measurement of temperatures and circulation of Titan's atmosphere [20]. Other targets will also be observed such as Saturn, other satellites and the rings but the investigation of Titan drives the design of the Radar and is the priority for the instrument. Special attention will be given to the imaging of Titans polar regions which experience a great amount of seasonal change so if evidence of surface liquids were to be present, they would be found near the poles. Attention will also be given to imaging the general population of crater. While only an estimated $25 \%$ of Titan's surface will be imaged with SAR, enough global coverage should be captured to produce a representative picture of Titan's craters which will help determine Titan's surface age.

\subsubsection{Cassini Plasma Spectrometer (CAPS)}

CAPS is composed of three instruments, the Electron Spectrometer (ELS), the Ion Beam Spectrometer (IBS) and the Ion Mass Spectrometer (IMS) [21]. The ELS measures electron velocity with respect to energy for a global survey of plasma density and temperature. Cassini spent $50 \%$ of its mission within Saturn's magnetosphere and 50\% in the solar wind and Saturn's magnetosheath contributing to an 
unprecedented study of the interaction between the two. The IBS provides high angular resolution measurements of incoming ions for solar wind measurements and ion ram flux determination at Titan. The IMS measures the mass to charge ratio of incoming ions and is designed to identify isobaric molecular species.

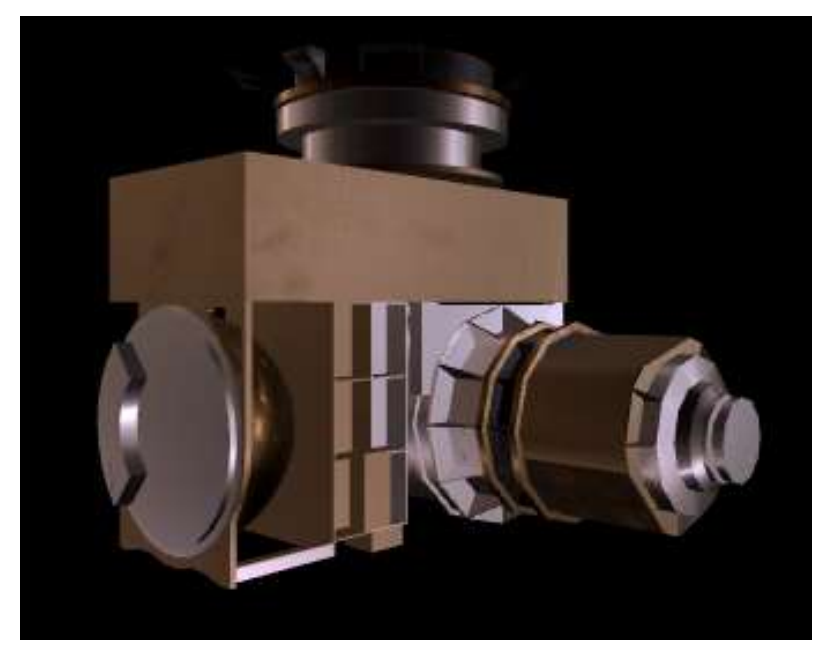

Figure 16. CAPS Instrument.

Table 7. CAPS Specifications [21].

\begin{tabular}{|c|c|c|c|c|}
\hline & \multicolumn{2}{|c|}{ IMS } & ELS & IBS \\
\hline Energy Range & \multicolumn{2}{|c|}{$1-50,280 \mathrm{eV} / \mathrm{e}$} & $0.6-28,750 \mathrm{eV} / \mathrm{e}$ & $1-49,8000$ \\
\hline Energy Res & \multicolumn{2}{|c|}{$0.17 \Delta \mathrm{E} / \mathrm{E}$} & $0.17 \Delta \mathrm{E} / \mathrm{E}$ & $0.014 \Delta \mathrm{E} / \mathrm{E}$ \\
\hline IFOV & \multicolumn{2}{|c|}{$8.3 \times 160^{\circ}$} & $5.2 \times 160^{\circ}$ & $1.4 \times 150^{\circ}$ \\
\hline Angular Res & \multicolumn{2}{|c|}{$8.3 \times 20^{\circ}$} & $5.2 \times 20^{\circ}$ & $1.4 \times 1.5^{\circ}$ \\
\hline & High Res & Low Res & & \\
\hline Mass Range & $1-400 \mathrm{amu} / \mathrm{e}$ & $1-100 \mathrm{amu} / \mathrm{e}$ & N/A & N/A \\
\hline \multirow[t]{2}{*}{ Mass Res } & $8 \mathrm{M} / \Delta \mathrm{M}$ & $60 \mathrm{M} / \Delta \mathrm{M}$ & N/A & N/A \\
\hline & \multicolumn{4}{|c|}{ Total } \\
\hline Mass & \multicolumn{4}{|c|}{$23.03 \mathrm{~kg}$} \\
\hline Power & \multicolumn{4}{|c|}{$16.4 \mathrm{~W}$} \\
\hline Data Rate & \multicolumn{4}{|c|}{$0.5-16 \mathrm{kbps}$} \\
\hline
\end{tabular}

The two primary investigation areas of the Caps instrument were Saturn's Magnetosphere and Titan's interaction with it. Within Saturn's magnetosphere, plasma sources and sinks as well as auroral processes were determined. Titan's interaction with Saturn's magnetosphere offered a case study of the interaction between a non-magnetized body with energized magnetic particles. The interaction between the two is like 
the planets' interaction with the Sun's magnetosphere allowing for an unprecedented study not capable anywhere else in the solar system.

\subsubsection{Cosmic Dust Analyzer (CDA)}

The Cassini Dust Analyzer studies the mass, impact rate, distribution, composition, electric charge, speed and flight direction of dust particles in the Saturn System [22]. The CDA consists of a high rate detector and a dust analyzer. The dust analyzer contains multiple grids of stainless steel stacked on top of one another and create a voltage when impacted by a dust particle. As the particle passes through the grids, the velocity, charge and mass are measured. The high rate detector consists of two polyvinylidene sensors that measure gross impacts per second when the dust analyzer is overwhelmed.

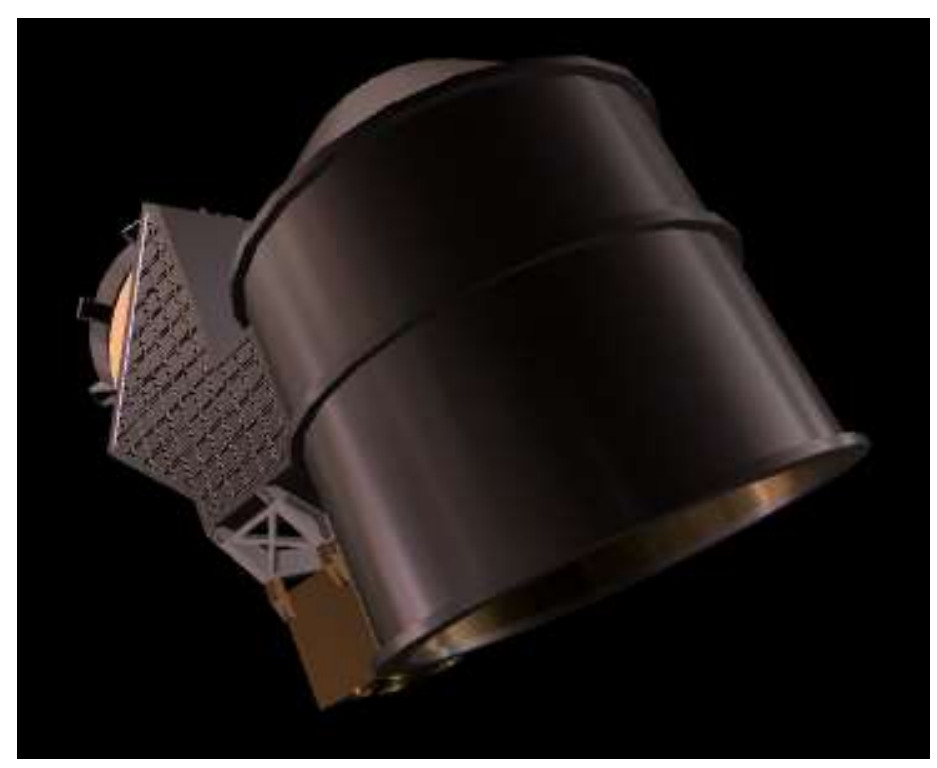

Figure 17. CDA Instrument.

Table 8. CDA Specifications [22].

\begin{tabular}{|l|l|}
\hline Mass Sensitivity & $5 \times 10^{-19}$ \\
\hline Max Detection Rate & $10^{4}$ impacts s $^{-1}$ \\
\hline Angle Accuracy & $5^{\circ}$ \\
\hline & \multicolumn{2}{|l|}{} \\
\hline Mass & $17.15 \mathrm{~kg}$ \\
\hline Power & $17.3 \mathrm{~W}$ \\
\hline Data Rate & $524 \mathrm{bps}$ \\
\hline
\end{tabular}


The CDA investigated three areas at Saturn; the rings, satellites, and magnetosphere. Within the rings the CDA mapped the ring distribution and searched for ring particle outside of the E ring. It also analyzed the ring particle composition. For the satellites, the CDA investigated the interaction between the ring particles and the satellites themselves. In the magnetosphere, the CDA worked in tandem with CAPS to investigate dust particles as sources and sinks for charged particles.

\subsubsection{Ion and Neutral Mass Spectrometer (INMS)}

The Ion and Neutral Mass Spectrometer is a quadrupole mass spectrometer that contains two ion sources for sampling ambient ions and neutral particles [23]. A quadrupole mass spectrometer contains a chamber of four rods connected to RF sources arranged in such a way to allow particles with a select charge through to the detection chamber. Within the detection chamber an electric field exists such that the charged particle is forced to impact the detector. Depending on where the particle impacts and the charge allowed through, the mass and actual charge can be determined. The ion sources work to charge any neutral particle that may enter the spectrometer.

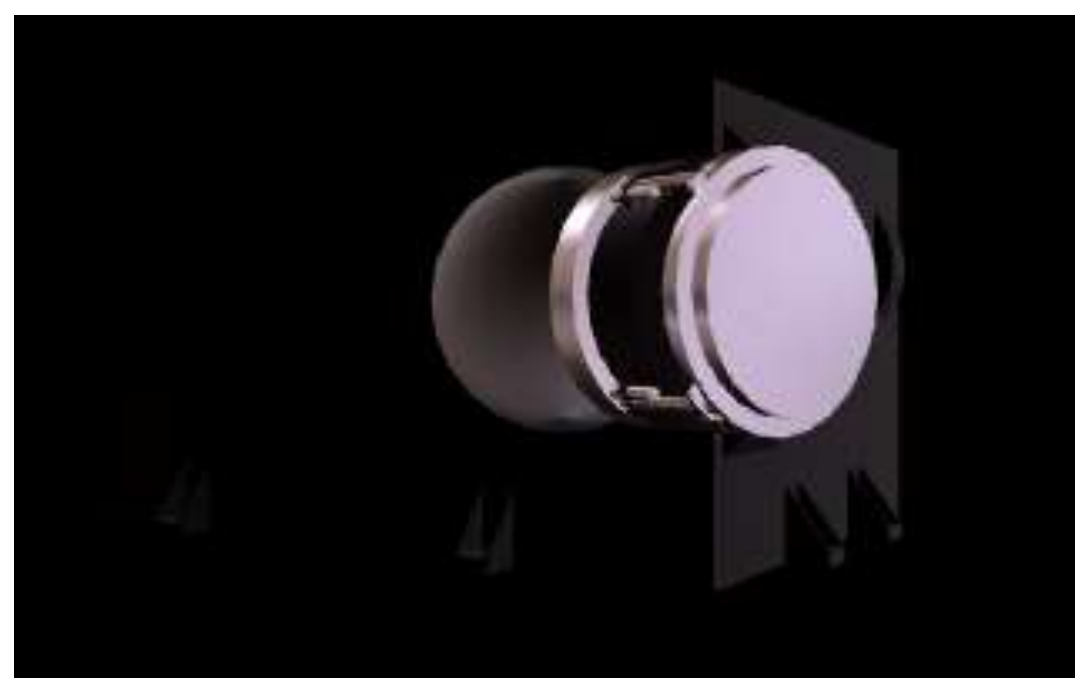

Figure 18. INMS Instrument. 
Table 9. INMS Specifications [23].

\begin{tabular}{|l|l|}
\hline View Angle & $8.6^{\circ}$ \\
\hline Mass Range & $1-8,12-99$ Daltons \\
\hline Resolution & $10^{-6}$ \\
\hline & \\
\hline Mass & $10.29 \mathrm{~kg}$ \\
\hline Power & $23.3 \mathrm{~W}$ \\
\hline Data Rate & $1510 \mathrm{bps}$ \\
\hline
\end{tabular}

The primary science objective of INMS was to characterize the composition and structure of Titans atmosphere. Specifically, INMS gathered data on the composition, density and temperature of the upper neutral atmosphere and the density and composition of the ionosphere. Determination of the formation of complex hydrocarbons was emphasized. INMS also investigated the neutral and plasma environments of Saturn's rings. The thermal plasma observed by Pioneer 11 is believed to be sourced from the rings so INMS sought to determine if this is the case.

\subsubsection{Magnetometer (MAG)}

The MAG instrument is composed of a fluxgate magnetometer (FGM) and a vector/scalar helium magnetometer (VHM/SHM). The helium magnetometer is placed at the tip of an $11 \mathrm{~m}$ boom with the fluxgate magnetometer located halfway between the spacecraft and the helium magnetometer. The distance provided by the boom reduces the field contamination from the electrical wiring of the space craft and the configuration of the magnetometers allows the field from the spacecraft to be calculated and calibrated out. 


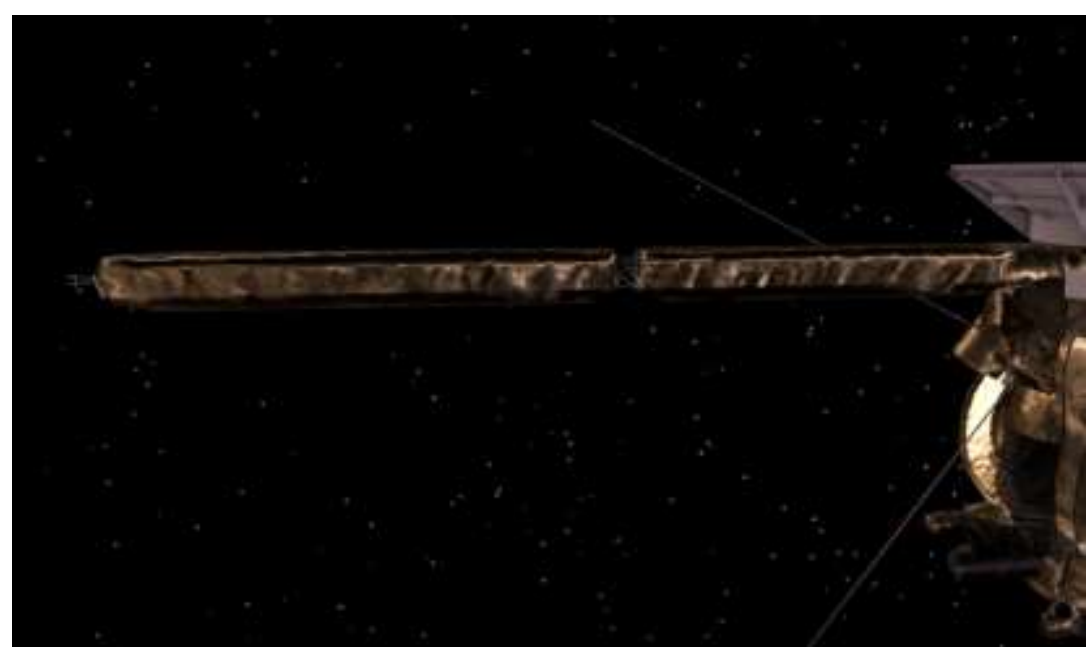

Figure 19. Cassini MAG Boom.

Table 10. MAG Specifications [24].

\begin{tabular}{|l|l|l|l|}
\hline & FGM & VHM & SHM \\
\hline Dynamic Range & $\pm 40 \mathrm{nT}$ & $\pm 32 \mathrm{nT}$ & $256-16,384 \mathrm{nT}$ \\
& $\pm 400 \mathrm{nT}$ & $\pm 256 \mathrm{nT}$ & \\
& $\pm 10,000 \mathrm{nT}$ & & \\
& $\pm 44,000 \mathrm{nT}$ & $36 \mathrm{pT}$ \\
\hline Resolution & $4.9 \mathrm{pT}$ & $3.9 \mathrm{pT}$ & \\
& $48.8 \mathrm{pT}$ & $31.2 \mathrm{pT}$ & \\
& $1.2 \mathrm{nT}$ & & \\
& $5.4 \mathrm{nT}$ & Total & \\
& & $8.82 \mathrm{~kg}$ & \\
\hline & & $12.63 \mathrm{~W}$ \\
\hline Mass & & $2000 \mathrm{bps}$ \\
\hline Power & & \\
\hline Data Rate & & \\
\hline
\end{tabular}

The MAG instrument conducted a global survey of magnetic field strengths and directions. Detailed readings will enable the internal planetary magnetic field to be resolved to the fourth order. The internal field will provide information about the dynamo region and the source of Saturn's magnetic field.

\subsubsection{Magnetospheric Imaging Instrument (MIMI)}

The Magnetospheric Imaging Instrument consists of three detector heads; the Ion and Neutral Camera (INCA) the Charge Energy Mass Spectrometer (CHEMS) and the Low Energy Magnetospheric Measurements System (LEMMS) [25]. The three detector heads perform a broad spectrum of 
investigations but are all connected by the same electronics. INCA is a time of flight detector that analyzes direction and motion of impacting energetic atoms to construct an image of the magnetosphere. CHEMS measures the distribution of elemental and molecular ions to characterize suprathermal ion populations.

CHEMS has a large view factor and when rolled about the Spacecraft can measure complete 3-D distributions. LEMMS measures the distribution of energetic ion and electron fluxes. The instrument head rotates about the y-axis of the spacecraft but can only obtain a 3-D measurement if Cassini is rotating about its z-axis.

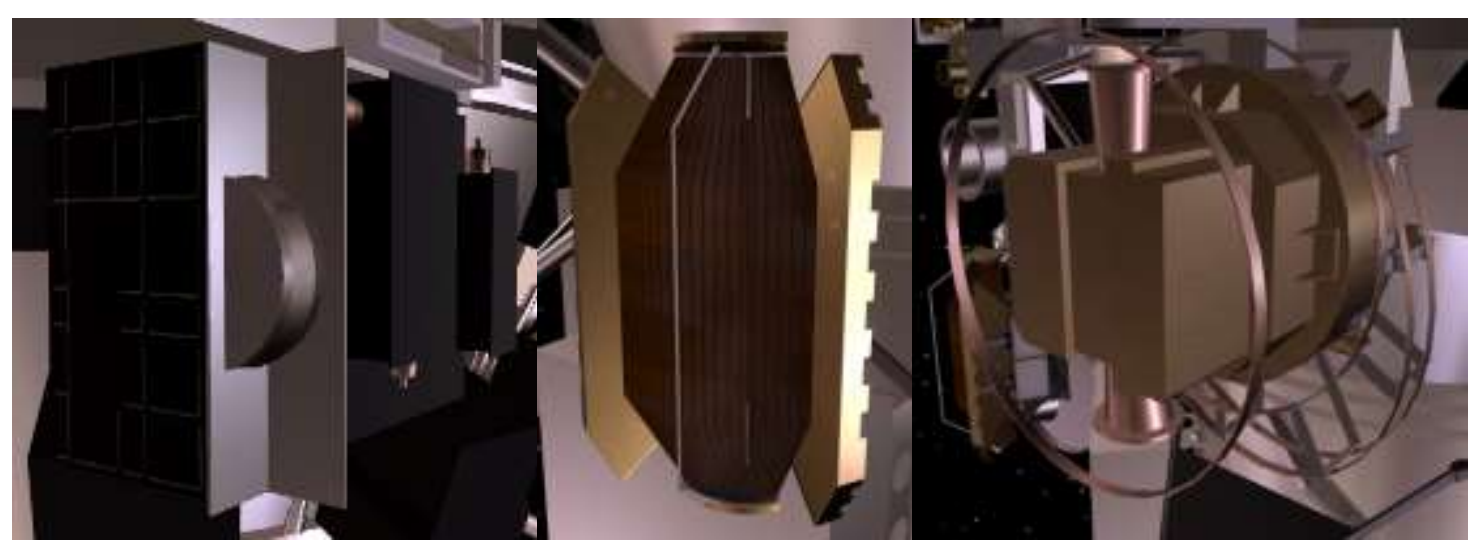

Figure 20. MIMI Detector Heads; CHEMS, INCA, LEMMS.

Table 11. MIMI Specifications [25].

\begin{tabular}{|l|l|l|l|}
\hline & INCA & CHEMS & LEMMS \\
\hline Energy Range & $<7 \mathrm{keV}-3 \mathrm{MeV} / \mathrm{nuc}$ & N/A & $\begin{array}{l}\text { Ions: } 0.03-160 \mathrm{MeV} \\
\text { Electrons: } 0.015-5 \mathrm{MeV}\end{array}$ \\
\hline Velocity Resolution & $<50 \mathrm{~km} / \mathrm{s}$ & N/A & N/A \\
\hline Mass Resolution & $\mathrm{H}, \mathrm{O}, \mathrm{Heavies}$ & N/A & N/A \\
\hline Field of View & $120^{\circ} \times 90^{\circ}$ & $4^{\circ} \times 159^{\circ}$ & N/A \\
\hline Energy/Charge Range & N/A & $3-220 \mathrm{keV} / \mathrm{e}$ & N/A \\
\hline Mass/Charge Range & N/A & $1-80 \mathrm{amu} / \mathrm{e}$ & \\
\hline & & \multicolumn{2}{|c|}{ Total } \\
\hline & & $28.11 \mathrm{~kg}$ \\
\hline Mass & & $20.3 \mathrm{~W}$ & \\
\hline Power & & & \\
\hline
\end{tabular}

The three detector heads will measure 3-D distributions, compositions and charge state of energetic particles within Saturn's magnetosphere. The measurements will be used to create images of the 
magnetosphere on a global scale. The images and 3-D distribution measurements will determine the configuration and dynamics of Saturn's magnetospheric plasma as well as its origins and interactions with satellites and rings.

\subsubsection{Radio and Plasma Wave Science (RPWS)}

The Radio and Plasma Wave Science consists of three orthogonal $10 \mathrm{~m}$ antennas to measure electric fields, three orthogonal search coil magnetometer antennas and a Langmuir probe [26]. The antennas are connected to receivers to measure a wide range of waveforms and frequencies associated with different wave sources.

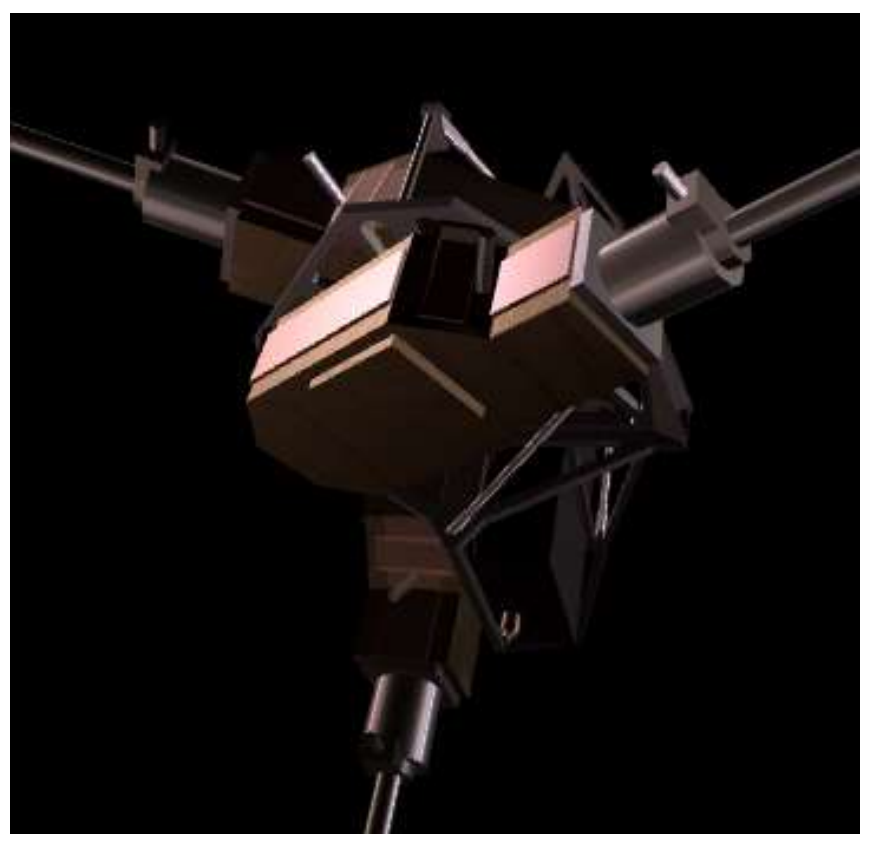

Figure 21. RPWS Instrument.

Table 12. RPWS Specifications [26].

\begin{tabular}{|l|c|c|l|}
\hline & Electric Antennas & Magnetic Antennas & Langmuir Probe \\
\hline Frequency Range & $1 \mathrm{~Hz}-16 \mathrm{MHz}$ & $1 \mathrm{~Hz}-12 \mathrm{kHz}$ & $1 \mathrm{~Hz}-2.5 \mathrm{kHz}$ \\
\hline Mass & & Total \\
\hline Power & & $37.68 \mathrm{~kg}$ \\
\hline
\end{tabular}


Saturn is one of five planetary sources of nonthermal radio emissions [26]. The electric antennas measure the frequency and strength of Saturn's emissions. The radio frequencies are governed in large part by the global magnetic field so measuring them will aid in the investigation of Saturn's interior magnetic dynamo.

The electric antennas also measure the frequencies of electrostatic discharges emanating from equatorial lighting storms. The measurement of the frequencies of these discharges enables the electron density in the upper atmosphere to be calculated. In addition, the antennas measure the impacts of micron scale dust. The measurements compliment those of the CDA in that the surface area of the antennas is much greater than the aperture of the CDA allowing for impact measurement in low area of low particle flux. The magnetic antennas measure electromagnetic fields and waves within Saturn's magnetosphere.

\subsubsection{Radio Science Instrument (RSI)}

The Radio Science Instrument uses the Cassini telecommunications system to perform a range of radio science. The instrument is unique in that part of the instrument is the DSN which is used as the detector for the transmitted radio frequencies. The RSI transmits at S, X, and Ka band using ultra stable oscillators separate from the normal telecommunication system to ensure precise transmission of the selected frequencies. The RSI also receives at X and Ka band for two-way calibration of the emitted frequencies. The frequencies can vary slightly depending the two-way calibration outcome but will around the frequencies given in Table 13.

Table 13. RSI Specifications [27] [8].

\begin{tabular}{|l|l|l|l|}
\hline & S band & X Band & Ka Band \\
\hline Frequency & $2.3 \mathrm{GHz}$ & $8.4 \mathrm{GHz}$ & $32 \mathrm{GHz}$ \\
\hline & & \multicolumn{2}{|c|}{ Total } \\
\hline & & $14.38 \mathrm{~kg}$ \\
\hline Mass & & $80.7 \mathrm{~W}$ \\
\hline Power & & \\
\hline
\end{tabular}

The two primary science areas of investigation of the RSI are gravity science and occultation experiments. Precision Doppler data obtained from the change in frequency received by the DSN is used to calculate global gravity maps of Saturn, Titan, and the icy satellites. The gravity field measurements are also used to accurately determine the mass of the bodies. Unlike the remote sensing instruments that use the Sun and 
stars as light sources to perform stellar occultation measurements, the RSI is the source of radio emissions that are then received and observed by the DSN. Transmission through the ionosphere and atmosphere also produce Doppler shifts of the frequency that are used to create vertical profiles of electron density as well as temperature, pressure and density of the atmosphere. Changes in the phase and amplitude of the transmitted signal are observed during occultations of the rings. The changes provide information on the radius and abundance of particles. 


\section{Architecture Selection}

In this section, several concept architectures using swarms of CubeSats are developed that would achieve the Cassini Primary mission's science objectives as well as similar data returns. A trade study was performed to choose the ideal architecture with emphasis was placed on architectures that will cost less than the Cassini mission. Potential spacecraft designs are developed for the selected architecture. Cost was prioritized while still meeting science objectives but overall data return was secondary to cost and science achieved. NASA is descoping the upcoming Wide Field Infrared Space Telescope to save \$400 million [28]. Many of the proposed architectures will save similar amounts or more compared to the original Cassini mission justifying the emphasis on cost in the selection process.

\subsection{Concept Exploration}

Several assumptions were made in creating the architectures; namely a mother spacecraft will be required to carry the CubeSats to Saturn and insert them into Saturn orbit, the mother spacecraft will follow Cassini's trajectory to Saturn and insert into the same initial orbit as Cassini, the mother and CubeSats have the opportunity to follow Cassini's primary mission trajectory within the Saturn system, the mother spacecraft will act as a telecommunication relay between the CubeSats and Earth, the Huygens probe will not need to be replicated, all of Cassini's instruments can be made sufficiently small to fit in a 6U CubeSat and still retain similar capabilities, the CubeSats would be able to survive the transit to Saturn and the total mission duration, the Mother and deployer will provide thermal and radiation protection during the transit to Saturn, and that RTGs are not available for use.

The main reason a mother was assumed to carry the CubeSats is that the high delta V necessary to enter Saturn orbit would be almost impossible for a CubeSat. Cassini needed about $600 \mathrm{~m} / \mathrm{s}$ to attain its initial orbit around Saturn [15] [29]. The mother also conveniently provides thermal and radiation protection due to the deployer throughout the interplanetary cruise. With the mother spacecraft needing a communications system to communicate with Earth and the mother being physically large due to the propellant it needs to carry. It was assumed that the mother would host a large high gain antenna and act as a relay for the CubeSats. For the sake of this study, optical communication systems were not considered. 
The Juno space probe was established as an initial baseline mother spacecraft hence forth referred to as the Mother. Juno has similar delta V capabilities as Cassini and has large solar arrays such that it doesn't require any RTGs. Juno had a wet mass of $3625 \mathrm{~kg}$ and dry mass of $1593 \mathrm{~kg}$ [30]. Juno had three solar array each $2.7 \times 9$ meters with a total area of just over $60 \mathrm{~m}^{2}$ due to one of the panels being shorter to accommodate a magnetometer boom [30]. Of the 60 square meters of solar panel arrays, 45 square meters had active solar cells producing approximately $475 \mathrm{~W}$ at $20 \%$ efficiency at Jupiter [30]. Juno uses a $645 \mathrm{~N}$ LEROS-1B engine with an Isp of 317 seconds [30] [31]. Given Juno's dry and wet masses as well as its main engine Isp, Juno's theoretical total delta V capability is calculated to be $2554 \mathrm{~m} / \mathrm{s}$ which is close to Cassini's theoretical capability of $2734 \mathrm{~m} / \mathrm{s}$ and greater than Cassini's needed delta V of $2040 \mathrm{~m} / \mathrm{s}$ [15].

The assumption of Cassini's instruments being mad sufficiently small was based on research performed at the Jet Propulsion Laboratory showing that CubeSat sized instruments are now completely feasible.

Because many of the instruments weren't feasible just five years ago but are now implies that further progress in miniaturization will only increase feasibility and performance. Figure 22 show an assessment of CubeSat based instruments and their feasibility.

\begin{tabular}{|c|c|c|c|}
\hline Technology & $\begin{array}{l}\text { Selva* and } \\
\text { Krejci, } 2012\end{array}$ & $\begin{array}{c}\text { Freeman } \\
2017\end{array}$ & Justification \\
\hline Atmospheric Chemistry Instruments & Problematic & Feasible & PICASSO, IR sounders \\
\hline Atmos Temp and Humidity Sounders & Feasible & Feasible & \\
\hline Cloud Profile and rain radars & Infeasible & Feasible & JPL RainCube Demo \\
\hline Earth Radiation Budget radiometers & Feasible & Feasible & SERB, RAVAN \\
\hline Gravity Instruments & Feasible & Feasible & Need a demo mission \\
\hline Hi-res Optical Imagers & Infeasible & Feasible & Planetlabs \\
\hline Imaging microwave radars & Infeasible & Feasible & Ka-Band $12 U$ design \\
\hline Imaging multi-spectral radiometers (Vis/IR) & Problematic & Feasible & AstroDigital \\
\hline Imaging multi-spectral radiometers ( $\mu$ Wave) & Problematic & Feasible & TEMPEST, \\
\hline Lidars & Infeasible & Feasible & DIAL laser occultation \\
\hline Lightning Imagers & Feasible & Feasible & \\
\hline Magnetic Fields & Feasible & Feasible & InSPIRE \\
\hline Multiple direction/polarization radiometers & Problematic & Feasible & HARP Polarimeter \\
\hline Ocean color instruments & Feasible & Feasible & SeaHawk \\
\hline Precision orbit & Feasible & Feasible & CanX-4 and -5 \\
\hline Radar altimeters & Infeasible & Feasible & Bistatic LEO-GEO \\
\hline Scatterometers & Infeasible & Feasible & GPS refl. (CyGNSS) \\
\hline
\end{tabular}

Figure 22. Feasibility of CubeSat Size Instruments [32]. 


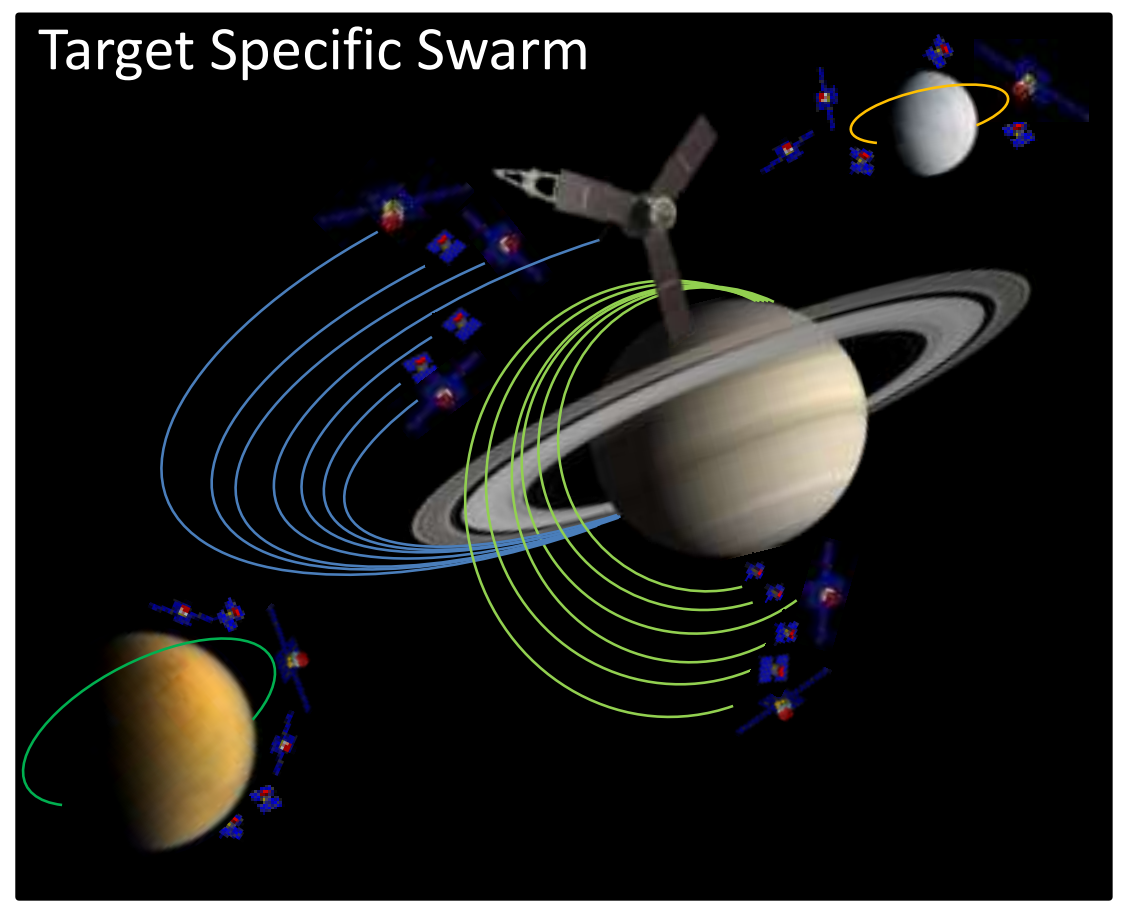

Figure 23. Target Specific Swarm Depiction.

The Target Specific Swarm, depicted in Figure 23, was the first architecture to be considered. The architecture consisted of sending swarms of CubeSats to each of the main scientific targets; Titan, Enceladus, rings, and polar regions. Sending swarms to individual targets would allow the swarms to be tailored and optimized for each target. The instruments carried would be specific to the science goals of specific target and could therefore be simplified due to their narrower scope and focus. All the CubeSats would be released after the Mother inserted into Saturn orbit and performed a phasing burn to raise its periapse. After deployment, the CubeSats would maneuver themselves using their own propulsion to achieve their respective orbits. The swarm investigating the polar region would use successive Titan gravity assists to change its orbit to eventually be inclined by $73^{\circ}$ to the equator. Cassini also used Titan gravity assists to change its inclination to $73^{\circ}$ so the swarm would not be increasing the mission time compared to Cassini by using gravity assists. The length of Cassini's four year tour was driven by the wide range of targets and the time taken traveling between. By having dedicated swarms for each target, the mission duration would likely be reduced by at least half. Reducing the mission to two years instead of four could 
save \$208 million since Cassini’s operations cost \$1.103 billion in 2017 dollars over nearly 11 years. On top of a reduction in mission duration, more data could be gathered from Titan and Enceladus due to target specific orbits to allow more flybys.

This architecture, while great for mission duration and scientific return, would be very complex from an operations standpoint and would likely be as expensive as the original Cassini mission. Both the high complexity and cost stem from the large total number of CubeSats required. Almost all the instruments collected data for each target requiring approximately 48 CubeSats for this architecture. Operating this many satellites poses an enormous challenge to mission operators. Very few constellations this size exist in orbit around Earth and none have ever flown in interplanetary space. While a few mega constellations have been proposed in Earth orbit such as One Web's 648 satellite constellation currently in development [33], Earth based constellations benefit from a multitude of ground station, tracking stations and the availability of the Global Positioning System. Most interplanetary spacecraft use the Deep Space Network for all their tracking and communication need. The DSN only has three sites around the world to serve all the interplanetary probes at a given time. NASA currently has only 12 active interplanetary probes [34] and so increasing the number of active satellites to above 60 would pose a huge logistical challenge to operate that many spacecraft simultaneously. Additionally, the design of the telecommunications subsystem for the Mother would be inherently complex in order to serve as the relay for as many as 48 individual CubeSats.

Tracking and navigation of the constellation would also be complex. While Cassini used the DSN for navigating to Saturn, once there it used the WAC to optically navigate within the Saturn System. The DSN can only provide $2.5 \mathrm{nrad}(1 \sigma)$ tracking accuracy at best which translates to $3.58 \mathrm{~km}$ position knowledge at Saturn distances [35]. This accuracy was insufficient for the Cassini navigation team and would most likely be insufficient for operating tight formations of CubeSats and for precise location knowledge to correlate data points to position in the Saturn system. Navigation techniques and hardware would need to be developed for the CubeSats in this architecture to overcome the inaccuracies of the DSN's tracking capabilities. Upgrades to the DSN's ground stations might bring its capabilities to an acceptable level but would require major investments to the sites. An optical navigation system could be developed for the CubeSats that uses positions of Saturn's moon for position determination. Such a system would likely 
require a dedicated navigation camera as but would also enable autonomous navigation reducing the operation complexity of this architecture.

The need for improved tracking and navigation abilities extends to all the CubeSat swarm architectures discussed, but the need is greatest and most complex for the Target Specific Swarm architecture due to its large number of CubeSats in different orbits with only one Mother.

The challenges of communicating with the CubeSats to supply commands and to receive data is also compounded by the large number of them. The design of the Mother's telecommunication system will be more complex with additional components to communicate with all the CubeSats. From an operations and tasking standpoint, timeslots will likely need to be allocated to each CubeSat for it to communicate with the mother. Separate channels with different frequencies could be established to enable simultaneous communication with multiple CubeSats but this would add mass and complexity to the telecommunication system. Cassini was only able to communicate with ground stations for 12 hours once a day due to the DSN's obligations to other missions [14]. A similar limitation of availability for communication support is assumed for all the architectures furthering the operational complexities, especially for the TSS architecture. The lack of continuous communication with the Mother will challenge the operations team to develop a schedule to communicate with all the CubeSats within the available time so a store and forward system will need to be developed.

The swarms could be released one at a time, after the previous swarm had completed its mission, to simplify tracking and communications. Doing so, however, would cause the mission duration to lengthen and likely be longer than the original Cassini mission. This would negate one of the primary benefits of this architecture and increase the cost by more than $\$ 208$ million which would likely outweigh any benefits of simplification.

All the complexities in navigating, tracking, operating and communications will add costs to this architecture. The largest cost however would be for the CubeSats themselves. Science grade interplanetary CubeSats are typically estimated to be $\$ 30$ million [36] which seems appropriate for this mission due to the likely complex CubeSats and high grade instruments and components. With TSS architecture's estimated 
48 CubeSats, the CubeSats alone will cost approximately $\$ 1.44$ billion. Some economy of scale could be realized manufacturing multiple similar CubeSats reducing the cost. However, the cost of building so many CubeSats would likely be several hundreds of millions of dollars which doesn't include the cost of the Mother. Accounting for the costs of complex operations tracking and navigation as well as the costs of the spacecraft, this architecture will likely be as expensive as the original Cassini mission. Even with cost sharing among other space faring nations, the cost of this architecture will likely still be very high for the prime coordinating organization.

While the cost of the TSS architecture defeat the original purpose of using swarms of CubeSats, it could provide more scientific return especially for key targets such as Titan and Enceladus. Additional returns could include global radar mapping of Titan, higher resolution imaging of the Tiger Stripe region of Enceladus and imaging of individual particles in the rings.

\subsubsection{Total Swarm (TS)}

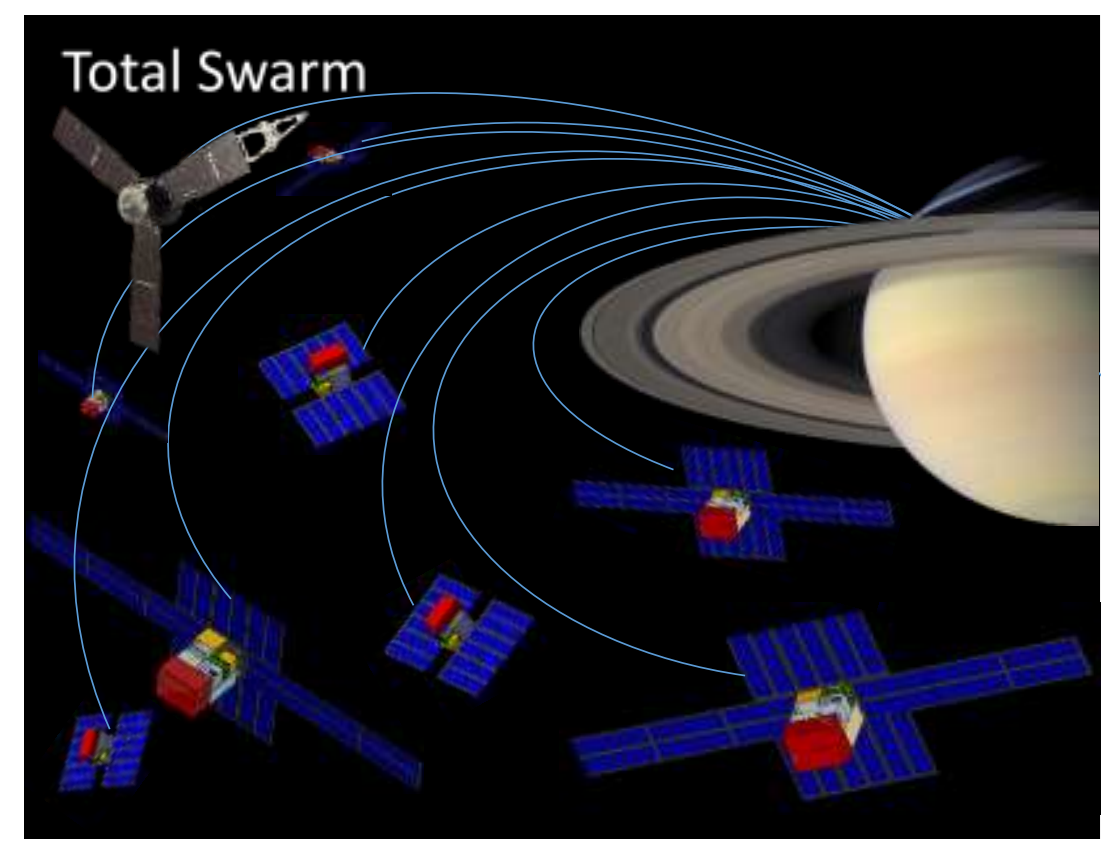

Figure 24. Total Swarm Depiction.

The Total Swarm architecture, depicted in Figure 24, involves removing all the Cassini instruments from the Mother except the Radar and RSS and moving the instruments into CubeSats. The Radar and RSS would remain on the mother and utilize the high gain telecommunication antenna in a similar set up as 
Cassini. Due to the size of the initial instruments, it is assumed that each instrument will get its own $6 \mathrm{U}$ CubeSat. The swarm of CubeSats would fly in close proximity to the Mother with the whole formation following Cassini's trajectory over its four year tour. All the CubeSats would be released after the Mother inserted into Saturn orbit and performed a phasing burn to raise its periapse. This architecture is effectively identical to the Cassini mission but with a swarm of CubeSats containing each instrument instead of a single monolithic spacecraft.

The biggest advantage of this architecture is a substantial reduction in complexity for the Mother. Instead of interfacing with 10 different instruments with widely varying power, data and mechanical needs, the Mother simply has two instruments and ten standardized CubeSat deployers. The deployers all have simple and identical interfaces and very low power and data needs. During the tour, the Mother doesn't need to be continually pointing instruments allowing for the possibility of a spin stabilized configuration. Because each CubeSat powers itself during the tour, the Mother doesn't need to power instruments, reducing the power production requirements for the Mother.

Because the Mother hosts the Radar, the Mother will need to actively point it, requiring the Mother to despin for each of the Titan flybys. The Mother would need to de-spin regardless of the Radar since after each flyby was when Cassini performed trajectory correction burn. There wouldn't be a benefit to having a CubeSat Radar as the Mother would need to de-spin anyways so the CubeSat wouldn't reduce any of the Mother's requirements.

With reduced instruments to power, spin stabilization and no power-hungry reaction wheels, it is feasible for the Mother to be solar powered. A mother with similar solar panel area as the Juno spacecraft utilizing next generation Low Incidence and Low Temperature solar cells [37] could generate as much power as 240 W at Saturn. Using new Roll Out Solar Array technology which is 20\% lighter [38] than traditional solar arrays like the ones used on Juno, $290 \mathrm{~W}$ could be produced at Saturn for the same mass as Juno's solar arrays. Using solar arrays instead of RTGs, which were used on Cassini, reduces the complexity of the Mother as well as the systems engineering and environmental analysis requirements all enabling a reduction in cost. 
While the operations of this architecture will be far simpler compared to the TSS, operating 10 CubeSats plus the Mother in formation is still difficult. The benefit of having only one swarm that moves together with the mother is the ease of disseminating commands and receiving data. Flying in close proximity could allow the CubeSats to communicate with the Mother through the Mother's omni directional low gain antennas. Doing so allows the Mother to maintain an Earth pointing orientation and builds the case for spin stabilization. The close proximity also allows for extremely high data rates between the Mother and the CubeSats minimizing time required in contact with each other. Complexities still exist with scheduling communication times with each CubeSat but ten CubeSats with high data rates is more reasonable than the TSS architecture.

Navigating the CubeSats will still require the development of a tracking system which likely can't be solved through upgrades to the DSN. A system of relative navigation will need to be developed to enable formation flight with the CubeSats staying within approximately $1 \mathrm{~km}$ of the Mother. If the spacecraft are separated by much more than $1 \mathrm{~km}$, it will be difficult for the formation to stay together over successive Titan Flybys [39]. The close proximity of the formation could allow for radio frequency based tracking similar to the DSN and the Electra radio system used on Mars orbiters and rovers. While a CubeSat communicates with the Mother, the Mother can measure the time delay in signals and Doppler shift in frequency to calculate range and range rate between the two spacecraft. From the range and range rate, position and velocity of the CubeSat relative to the mother could be determined. The Mother would likely serve as the hub for this type of tracking system to reduce the processing power needed by the likely power and volume constrained CubeSats. The development of such a tracking system is not covered in the scope of this paper and is only mentioned here as a proof of feasibility as the Electra radio operates on the same principles but in UHF frequencies.

The hardware cost for this architecture will be far lower. Reducing the number of CubeSats from 48 to 10 reduces costs by as much as $\$ 1.14$ billion. The Mother will also likely be cheaper with its simpler system for tracking and communicating with the CubeSats. This architecture will add two years to the mission adding \$208 million for extends operations. Even with the added operation costs, the Total Swarm architecture would be as much as $\$ 900$ million cheaper than the Target Specific Swarm. 
The costs for the ten CubeSats would still be fairly expensive at $\$ 300$ million. But the use of CubeSats opens the door to collaboration with other nations and space agencies. The Mother could be provided by the primary agencies with the CubeSats contributed by new and emerging space faring nations with national space agencies such as Australia, United Arab Emirates, Vietnam, Poland, Nigeria, Argentina, and South Korea or even more established players such as Japan, Canada, Russia, ESA, United Kingdom, France, Italy and Germany. These nations and their space agencies could reasonably contribute a CubeSat given the number of commercial CubeSat developers around the world and the founding premise of CubeSats as simple, low cost spacecraft. The potential gain of geopolitical prowess in having contributed an entire spacecraft to a flagship interplanetary mission and reasonable budget of $\$ 30$ million would likely be sufficient to attract many agencies for the project.

\subsubsection{Remote Sensing Swarm (RSS)}

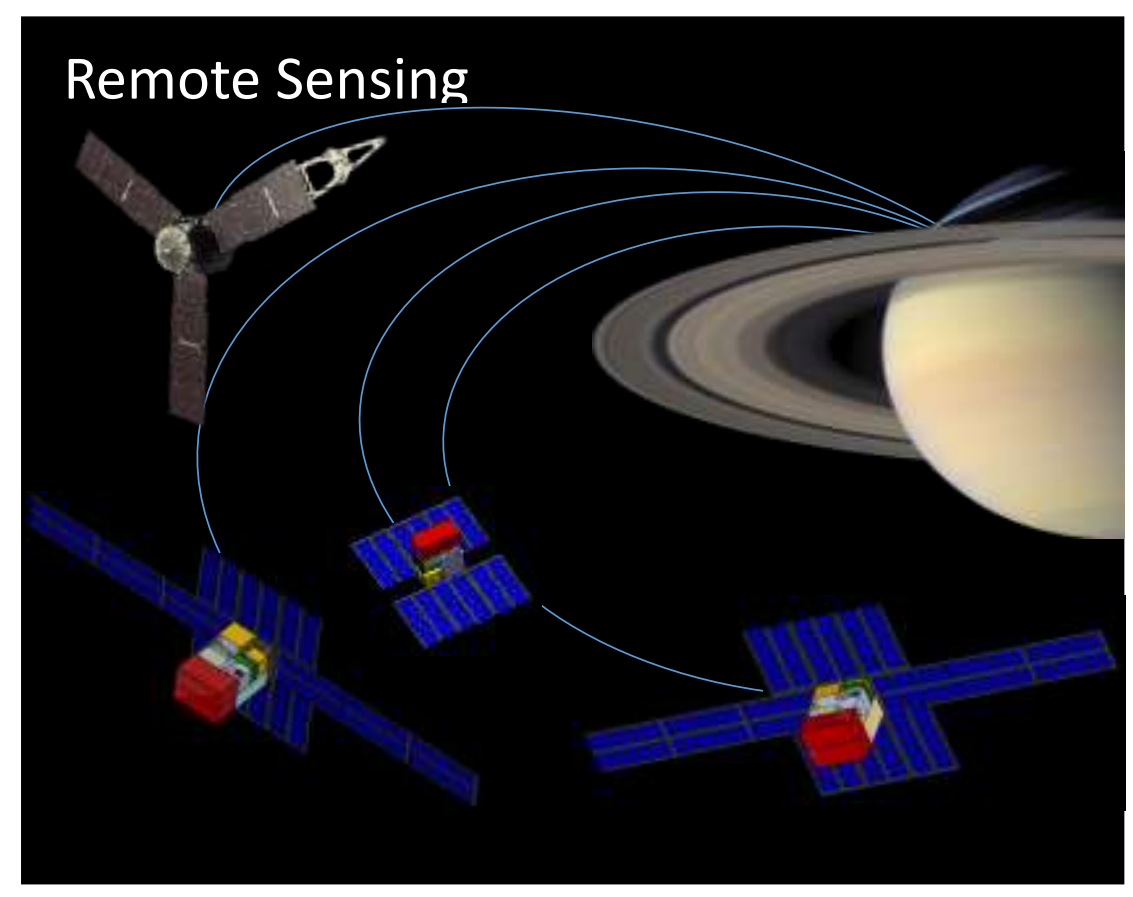

Figure 25. Remote Sensing Swarm Depiction.

The Remote Sensing Swarm architecture, depicted in Figure 25, only consists of the remotes sensing instruments (ISS, VIMS, UVIS and CIRS) being moved to CubeSats while the remaining instruments remain on the Mother. For this architecture, like the Total Swarm, the CubeSats and the Mother follow 
Cassini's same trajectory for the four year Primary Mission. The RSS architecture attempts to reduce some of the complexities of the TS architecture by reducing the Number of CubeSats. Additionally, because the fields and particles instruments are passive, there is no scientific benefit to moving them off the Mother onto CubeSats. By keeping the fields and particles instruments on the Mother, the total number of CubeSats is reduced to four. Scheduling communication and tracking times inherently becomes simpler and communications could become more frequent reducing the amount of storage the CubeSats need as well as performing tracking and navigations activities more often to increase position knowledge.

Reducing the number of CubeSats will also lower the hardware cost by approximately $\$ 150$ million assuming the instruments cost $\$ 5$ million and are simply placed on the mother. The Mother will cost slightly more due its increased engineering complexity of needing to supply power to the instruments as well as command and receive data from them. The Total cost of this architecture would likely be around $\$ 100$ million less than the TS architecture due to the added design complexities of the Mother. Even though the total mission cost is less, if a cost sharing strategy of having other organizations contribute the CubeSats is implemented, the primary organizing agency could actually see a slight increase in cost due to the increased Mother Cost.

The solar panels will likely need to grow to accommodate the increased power consumption. The Cassini fields and particles instruments together consumed a total of $85 \mathrm{~W}[6]$ and assuming that at worst case the Mother would need to supply the same power, the solar panel area would need to grow by $15.8 \mathrm{~m}^{2}$. The active solar panel area for Juno was $45 \mathrm{~m}^{2}$ with a mass of $340 \mathrm{~kg}$ [40] so to accommodate the increase in power, the Juno solar panels would increase in mass by $\% 33$ or $113 \mathrm{~kg}$. Modern instruments would likely require less power and using roll out solar arrays would both reduce the mass growth of the solar panels. Eliminating six CubeSat deployers and the CubeSat hardware will also offset solar panel mass increases. Assuming each CubeSat has a mass of $12 \mathrm{~kg}$ which is the maximum allowable mass for a $6 \mathrm{U}$ [41] [42] and the use of a Planetary Systems Corporation 6U deployer which has a mass of $4.5 \mathrm{~kg}$ [43] and that the payloads have a mass less than $4 \mathrm{~kg}, 75 \mathrm{~kg}$ is saved be keeping the fields and particles instruments on the Mother. At first glance, any mass increase in the mother would likely be minimalized by the reduced CubeSat mass. 
Aside from the reduced cost and operational complexity of this architecture, an advantage of this architecture is the increased reliability. With fewer CubeSats and therefore fewer components, the probability of a failure decreases.

\subsubsection{Multi Total Swarm (MTS)}

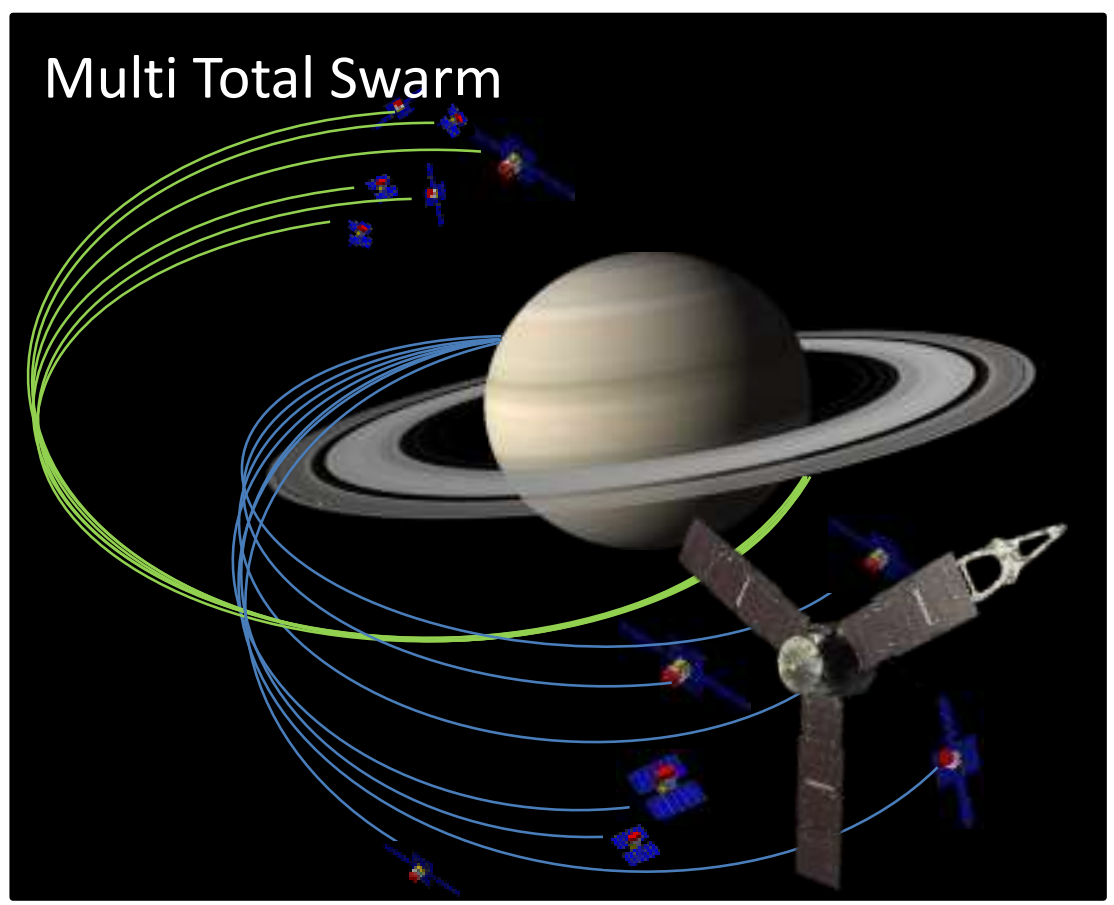

Figure 26. Multi Total Swarm Depiction.

The Multi Total Swarm architecture, depicted in Figure 26, consists of a Mother accompanied by two swarms of CubeSats each containing all the Cassini instruments except the Radar and RSI which would stay on the Mother and utilize its high gain antenna. One Swarm will be in a highly inclined investigate the aurora and other plasma and magnetospheric phenomena. The other Swarm will stay in Saturn's equatorial region to investigate Titan, Enceladus, and the rings. All the CubeSats would be released after the Mother inserted into Saturn orbit and performed a phasing burn to raise its periapse. After deployment, the CubeSats would maneuver themselves using their own propulsion to achieve their respective orbits. The swarm investigating the polar region would use successive Titan gravity assists to change its orbit to eventually be inclined by $73^{\circ}$ to the equator. Cassini also used Titan gravity assists to change its inclination to $73^{\circ}$ so the swarm would not be increasing the mission time compared to Cassini by using gravity assists. 
The Mother would orbit in formation with the equatorial swarm. This architecture takes the best aspects of the TS and TSS architectures, simple Mother, opportunity for collaboration and cost sharing, and shortened mission duration.

The inclined swarm will likely need to use Titan flybys to enter an inclined orbit as the amount of fuel the CubeSats can carry will not be enough to perform an inclination change maneuver and still have enough propellant left for multiple years' worth of orbital corrections. By having a swarm in a polar orbit and another in an equatorial orbit, the mission duration can be reduced by roughly half. The Cassini tour took the spacecraft through both inclined and equatorial orbits to investigate the entire Saturn system. By reducing the mission duration, operation costs can be saved. Conversely, for the same mission duration, more science investigations can be performed and more data points can be gathered for more temporal resolution of different phenomena.

By having multiple swarms, operations become more difficult. The additional CubeSats reduce the duration of communications all the CubeSats have with the Mother. Flexibility is also lost to troubleshoot potential issues or handle any unexpected events or scientific opportunities. Scheduling communications is more complex and requires greater forethought due to the limited time of communication. Cassini's nine hours of contact with Earth would be shared by 20 CubeSats as opposed to 10 in the TS architecture or four in the RSS architecture. With the reduced time spent in communication with each CubeSat, tracking and position accuracy will likely decrease. No matter what tracking system is implemented, less time spent tracking the CubeSats will result in worse position accuracy. Depending on requirements for position knowledge, the reduced tracking time available could result in a more complex tracking system. Conceivably a system could be developed to communicate with and track multiple CubeSats simultaneously but such a system would require multiple channels at different frequencies adding complexity and mass to the Mother.

The inclined swarm would also be at a much greater distance from the Mother as compared to the equatorial CubeSats in the immediate vicinity of the Mother. The increased distance will reduce the data rate at which the inclined swarm CubeSats can communicate with the Mother requiring more time to relay data. Additionally, the data rate at which the Mother will need to communicate with the Earth will double to still return the same amount of data for a condensed two year mission. Cassini required one gigabit of 
data to be relayed per day over a 12 hour communication window [14]. For a full four year mission, 1 gigabit over 12 hours translates to a data rate of $23 \mathrm{kbps}$. For a condensed 2 year mission, a data rate of 46 kbps is needed. To accommodate the increased data rate, the Mother's RF input power would need to double if the telecom system remained the same or the telecom system would need to change dish size or frequency. Either adding power or changing the telecom system will add mass and complexity. Increasing the data rate more than required would not decrease the mission duration, rather it would only increase the amount of data returned.

The overall cost of this architecture however, will be higher than both the TS and RSS architectures but still lower than the TSS architecture. With approximately 10 more CubeSats than the TS and RSS and each CubeSat costing an estimated $\$ 30$ million [44] or more, CubeSat costs for this architecture would likely be $\$ 300$ million more than the TS and RSS architectures not including the extra deployers or any added complexities to the Mother. Launch mass will also increase due to the additional CubeSats and deployers as well as the extra propellant required to carry them through the $1240 \mathrm{~m} / \mathrm{s}$ of delta $\mathrm{V}$ required to reach Saturn following Cassini’s trajectory [15]. Assuming the inclined swarm has ten 6U CubeSats, one for each instrument minus the Radar and RSS which would remain on the Mother, the inclined swarm will add 165 $\mathrm{kg}$ not including additional harnessing for the deployers. This assumes each CubeSat has a mass of $12 \mathrm{~kg}$ which is the maximum allowable mass for a $6 \mathrm{U}$ [41] [42] and the use of a Planetary Systems Corporation $6 \mathrm{U}$ deployer which has a mass of $4.5 \mathrm{~kg}$ [43]. The increase in mass could require the use of a more powerful launch vehicle further increasing costs. The price difference between an Atlas V 531 and 551 is $\$ 13$ million [45] and are quoted as costing \$160 million and \$173 million respectively for the two variants. If launch requirements exceed the capabilities of the Atlas V, the largest rocket in the U.S. fleet would be needed which costs almost $\$ 400$ million [46]. With the increases in complexity and mass of the Mother and the additional CubeSats while factoring the \$208 million savings from reduced mission length, it seems reasonable that this architecture would be $\$ 150$ million and \$250 million more than the TS and RSS architectures respectively.

While the increase in cost from the extra CubeSats could be mitigated through international partnerships, finding partners for 20 CubeSats would be challenging and using all non-US instruments would likely be 
unacceptable to NASA. More probable is that a few core participating agencies would supply multiple CubeSats which requires a greater financial commitment. Enough collaborating organizations could be found but the primary agency would need to supply some CubeSats in addition to the Mother.

\subsubsection{Remote Sensing and Total Swarm (RSTS)}

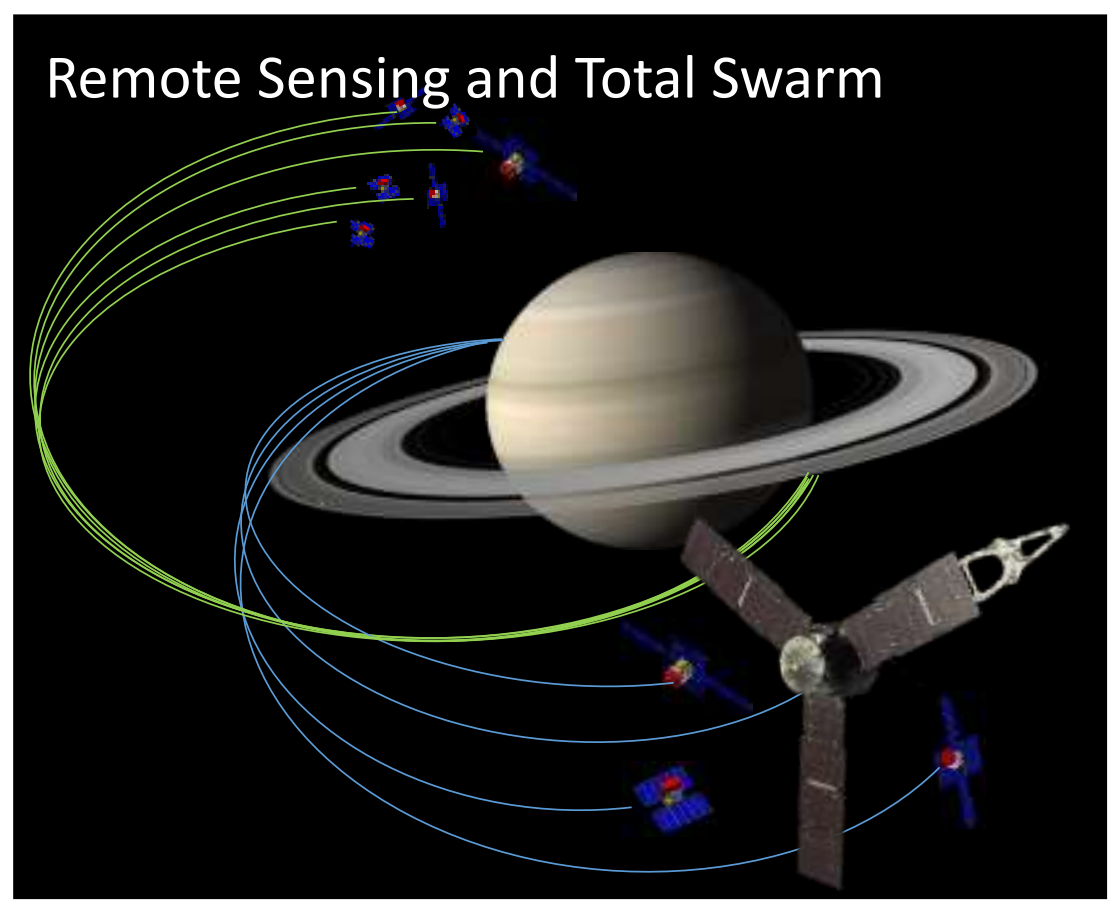

Figure 27. Remote Sensing and Total Swarm Depiction.

The Remote Sensing and Total Swarm architecture, depicted in Figure 27, consists of a Mother accompanied by a remote sensing swarm in an equatorial orbit and a separate swarm containing all the instruments in a highly inclined orbit. This architecture is nearly identical to the Multi Total Swarm architecture except it reduces the equatorial swarm to just the remote sensing instruments. The point of this architecture is to minimize the number of CubeSats to reduce cost and complexity while still achieving reduced mission length.

The equatorial swarm can be reduced to just the remote sensing instruments, ISS, VIMS, UVIS and CIRS because the rest of the instruments can be housed onboard the Mother. The inclined swarm still needs 10 CubeSats for all the instruments minus the Radar and RSS since those 10 instruments all performed investigations at high Saturn latitudes. 
Complexities still exist with having a separate inclined swarm. The distance between the Mother and inclined swarm at worst case could be as much as several Saturn radii with one radii being 60,268 $\mathrm{km}$ [47]. The distance will still affect communications and tracking for this architecture such that a reduction in complexity of the system responsible for these tasks will not diminish appreciably from the same system in the Multi Total Swarm architecture. The Mother however, like in the RSS architecture will become more complicated. The Mother will need to provide the fields and particles instruments with power as well as support them with command and data interfaces. Operations and scheduling will be easier with fewer spacecraft.

This architecture would save about $\$ 150$ million compared to the MTS architecture by reducing the number of CubeSats. With $\$ 150$ million in savings, this architecture would have the same cost as the TS but would still be $\$ 100$ million more than the RSS.

\subsubsection{Multi Mother Total Swarm (MMTS)}

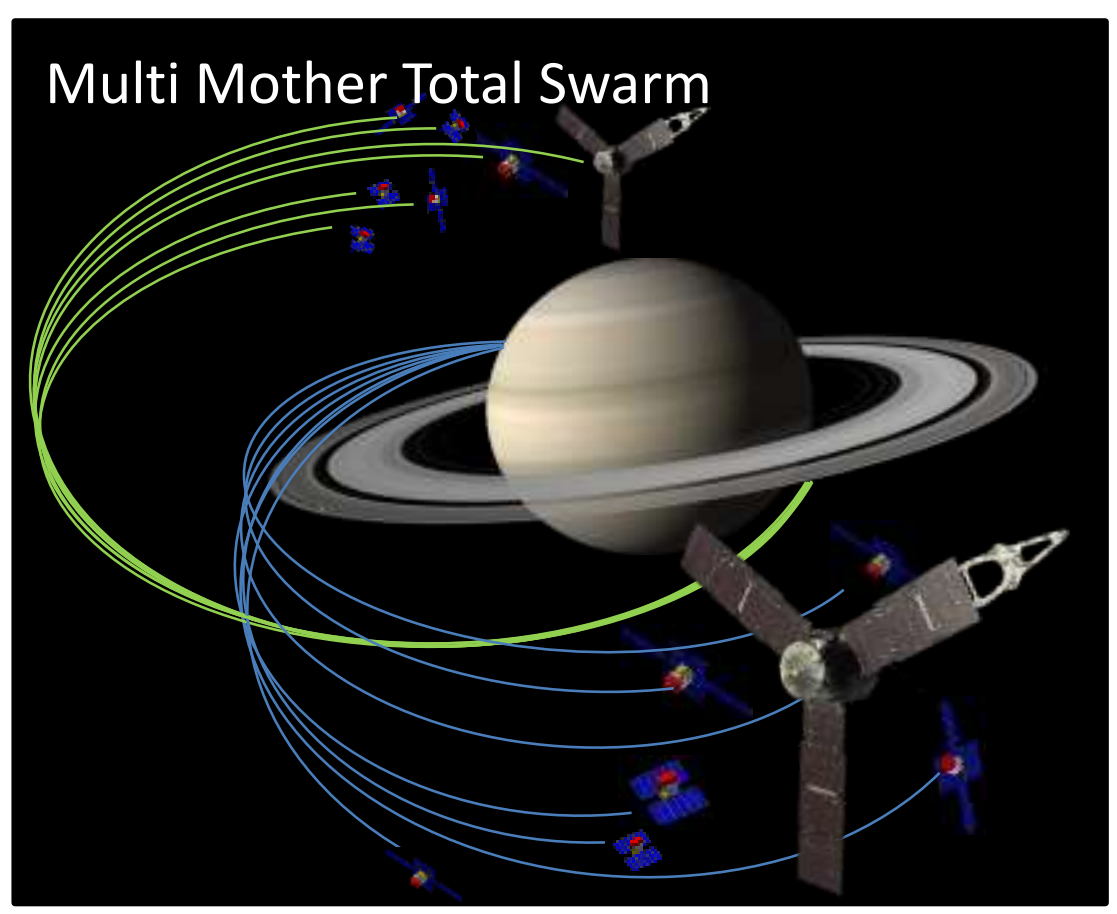

Figure 28. Multi Mother Total Swarm Depiction.

The Multi Mother Total Swarm, depicted in Figure 28, consists of two total swarms like the MTS architecture but has a mother accompany each swarm. The two swarms, one in an equatorial orbit and the 
other in a highly inclined orbit, will enable the mission duration to be reduced by half. Each swarm has all the instruments on the CubeSats except the Radar and RSI which stay on the Mother and utilize the Mother's high gain antenna. Moving the rest of the instruments off the Mother keeps the design simple like the TS architecture.

The idea behind this architecture is that is has all the advantages of the Total Swarm but has the reduced mission duration of the multi swarm architectures. Having a second Mother orbit in formation with the other swarm allows the Mother to be the hub for tracking, navigation and communication for that swarm. The two swarms could be almost identical simplifying design and manufacturing cost. However, adding a second Mother adds a tremendous amount of mass if the two swarms were launched on the same vehicle. The Mothers would also need to be stacked complicating their design. This architecture would most likely require the use of a Delta 4 Heavy which costs $\$ 400$ million. An Ariane 5 could be used and has the advantage of built in dual launch capability such that the two mother aren't stacked directly on each other. The Ariane 5 is less powerful than the Delta 4 Heavy so meeting its mass capability could prove challenging. The Ariane 5 is also a European rocket which might pose political issues unless ESA provides one like they did with the James Webb Space Telescope [48]. Between the more powerful launch vehicle and the addition of another Mother, this architecture will be very costly even with the simple design and reduced mission duration. 


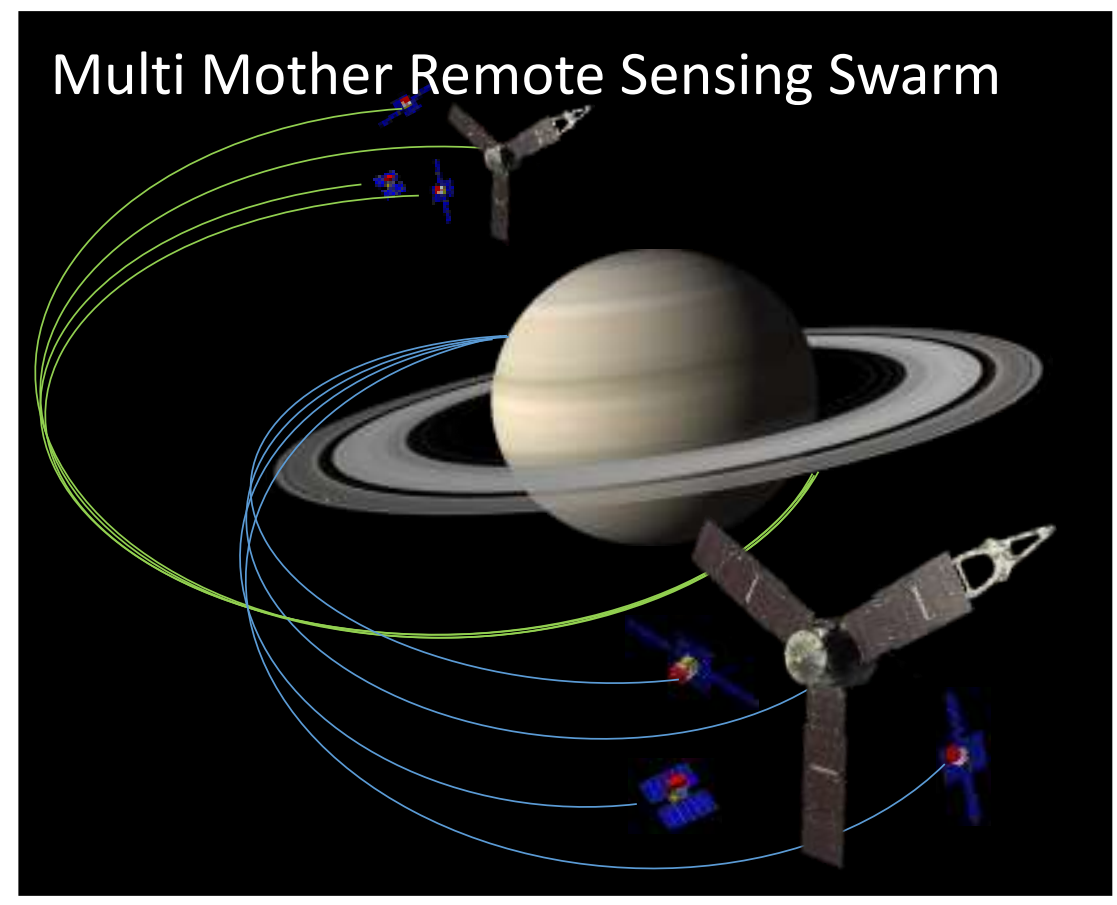

Figure 29. Multi Mother Remote Sensing Swarm Depiction.

The Multi Mother Remote Sensing Swarm architecture, depicted in Figure 29, uses two Mother spacecraft each with its own swarm of remote sensing CubeSats. One Mother with its swarm would have a highly inclined orbit to collect data at Saturn's poles. The other Mother and swarm orbit Saturn in its equatorial plane to investigate Saturn's satellites and rings. This architecture is near identical to the MMTS architecture but has two remote sensing swarms instead of total swarms. Just like the RSS architecture the MMRSS architecture seeks to reduce mission costs by reducing the number of CubeSats. This architecture would still have many of the challenges of the MMRS architecture and any cost savings derived from reducing the number of CubeSats will likely not be enough to justify the increased cost from the more powerful launch vehicle and construction of the second Mother.

\subsection{Concept Selection}

To select the best architecture a trade study was conducted. Each architecture was scored on four categories; Cost, Science Return, Complexity, and Reliability. Each category was weighted and summed to calculate a score with 5 being the highest possible. Each category was scored between 1 and 5 with 1 being 
the worst for that category and 5 being the best. Cost was weighted as $40 \%$ as the point of this study is to use CubeSats to reduce costs. Science return was weighted as $30 \%$ because the motivation of NASA missions is to gain scientific knowledge. Complexity was weighted as $20 \%$ because it will a determining factor in implementing CubeSat architectures at a programmatic level. Reliability was weighted as $10 \%$ because while an important consideration, the other categories are more important for this initial high level study. Tables Error! Reference source not found., Error! Reference source not found., Table 16, Error! Reference source not found., Error! Reference source not found., Error! Reference source not fou nd. and Table 20 summarize the characteristics of the architectures. Tables Table 21, Table 22, Table 23 and Table 24 outline how each architecture was scored and the reasoning behind the score. The trade Matrix and results are shown in Table 25.

Table 14. TSS Architecture Summary.

\begin{tabular}{|l|l|}
\hline \multicolumn{2}{|c|}{ TSS Architecture Summary } \\
\hline Number of Swarms & 4 \\
\hline Number of CubeSats & 48 \\
\hline Number of Mothers & 1 \\
\hline Mission Duration & $<2$ years \\
\hline Comments & $\begin{array}{l}\text { Each swarm contains all 12 instruments, one per CubeSat. Each swarm } \\
\text { targets a specific area of interest for Cassini }\end{array}$ \\
\hline
\end{tabular}

Table 15. TS Architecture Summary.

\begin{tabular}{|l|l|}
\hline \multicolumn{2}{|c|}{ TS Architecture Summary } \\
\hline Number of Swarms & 1 \\
\hline Number of CubeSats & 10 \\
\hline Number of Mothers & 1 \\
\hline Mission Duration & 4 years \\
\hline Comments & $\begin{array}{l}\text { Swarm and Mother fly in formation and follow Cassini's tour trajectory. } \\
\text { All instruments are placed on the CubeSats except the Radar and RSI } \\
\text { which use the Mothers antenna. Reduces number of CubeSats but } \\
\text { increases mission duration. }\end{array}$ \\
\hline
\end{tabular}

Table 16. RSS Architecture Summary.

\begin{tabular}{|l|l|}
\hline \multicolumn{2}{|c}{ RSS Architecture Summary } \\
\hline Number of Swarms & 1 \\
\hline Number of CubeSats & 4 \\
\hline Number of Mothers & 1 \\
\hline Mission Duration & 4 years \\
\hline Comments & $\begin{array}{l}\text { Swarm and Mother fly in formation and follow Cassini's tour trajectory. } \\
\text { Only the remote sensing instruments are placed on CubeSats. Minimizes } \\
\text { number of CubeSats but maintains simplicity of Mother. }\end{array}$ \\
\hline
\end{tabular}


Table 17. MTS Architecture Summary.

\begin{tabular}{|l|l|}
\hline \multicolumn{2}{|c|}{ MTS Architecture Summary } \\
\hline Number of Swarms & 2 \\
\hline Number of CubeSats & 20 \\
\hline Number of Mothers & 1 \\
\hline Mission Duration & 2 years \\
\hline Comments & $\begin{array}{l}\text { Two Total Swarms, one in a highly inclined orbit, one in an equatorial } \\
\text { orbit. Enables a reduced mission duration to save operations costs. }\end{array}$ \\
\hline
\end{tabular}

Table 18. RSTS Architecture Summary.

\begin{tabular}{|l|l|}
\hline \multicolumn{2}{|c|}{ RSTS Architecture Summary } \\
\hline Number of Swarms & 2 \\
\hline Number of CubeSats & 14 \\
\hline Number of Mothers & 1 \\
\hline Mission Duration & 2 years \\
\hline Comments & $\begin{array}{l}\text { One Total Swarm in an inclined orbit, one Remote Sensing Swarm and the } \\
\text { Mother in an equatorial orbit. Reduces number of CubeSats compared to } \\
\text { MTS. }\end{array}$ \\
\hline
\end{tabular}

Table 19. MMTS Architecture Summary.

\begin{tabular}{|l|l|}
\hline \multicolumn{2}{|c|}{ MMTS Architecture Summary } \\
\hline Number of Swarms & 2 \\
\hline Number of CubeSats & 20 \\
\hline Number of Mothers & 2 \\
\hline Mission Duration & 2 years \\
\hline Comments & $\begin{array}{l}\text { Two Total Swarms and two Mothers, one Mother for each swarm. One } \\
\text { swarm in a highly inclined orbit, one in an equatorial orbit. Enables a } \\
\text { reduced mission duration to save operations costs. Second Mother } \\
\text { simplifies communications and operations. }\end{array}$ \\
\hline
\end{tabular}

Table 20. MMRSS Architecture Summary.

Number of Swarms

Number of CubeSats

Number of Mothers

Mission Duration

Comments

\section{MMRSS Architecture Summary}

2

8

2

2 years

Two Remote Sensing Swarms and two Mothers, one Mother for each swarm. One swarm in a highly inclined orbit, one in an equatorial orbit. Enables a reduced mission duration and reduces the number of CubeSats. Second Mother simplifies communications and operations. 
Table 21. Cost Scoring.

\begin{tabular}{|l|c|l|}
\hline Architecture & Score & Reason \\
\hline TSS & 1 & $\begin{array}{l}\text { Most expensive architecture with total costs approaching the cost of the } \\
\text { original Cassini mission. Large number of CubeSats leads to large hardware } \\
\text { cost. }\end{array}$ \\
\hline TS & 4 & $\begin{array}{l}\text { Second cheapest architecture at } \$ 100 \text { million more than RSS. Small number } \\
\text { of CubeSats reduces hardware cost but has more CubeSats than the RSS } \\
\text { architecture. }\end{array}$ \\
\hline RSS & 5 & $\begin{array}{l}\text { Cheapest architecture. Reduces hardware costs by having the fewest } \\
\text { CubeSats while still simplifying the Mother }\end{array}$ \\
\hline MTS & 2 & $\begin{array}{l}\text { Large number of CubeSats increases cost to \$300 million more than RSS. } \\
\text { Second swarm at far distances adds hardware cost and requires more complex } \\
\text { and therefore expensive telecommunication system for the Mother. }\end{array}$ \\
\hline RSTS & 4 & $\begin{array}{l}\text { Middle ground pricing at \$100 million more than RSS. Reduced operation } \\
\text { time enough to make up for larger number of CubeSats. }\end{array}$ \\
\hline MMTS & 1 & $\begin{array}{l}\text { Expensive because of second Mother and likely need for a large and } \\
\text { expensive launch vehicle. }\end{array}$ \\
\hline MMRSS & 1 & $\begin{array}{l}\text { Expensive because of second Mother and likely need for a large and } \\
\text { expensive launch vehicle. }\end{array}$ \\
\hline
\end{tabular}

Table 22. Science Return Scoring.

\begin{tabular}{|c|c|c|}
\hline \multicolumn{3}{|r|}{ Science Return } \\
\hline Architecture & Score & Reason \\
\hline TSS & 5 & $\begin{array}{l}\text { Large number of CubeSats offer potential for large science return, potentially } \\
\text { more than Cassini returned because of detailed ring, moon and Titan study } \\
\text { opportunities. }\end{array}$ \\
\hline TS & 3 & Meets the Cassini science objectives but not more. \\
\hline RSS & 3 & Meets the Cassini science objectives but not more. \\
\hline MTS & 4 & $\begin{array}{l}\text { Has the potential to perform additional studies beyond Cassini but not as } \\
\text { many as TSS. }\end{array}$ \\
\hline RSTS & 4 & $\begin{array}{l}\text { Has the potential to perform additional studies beyond Cassini but not as } \\
\text { many as TSS. }\end{array}$ \\
\hline MMTS & 4 & $\begin{array}{l}\text { Has the potential to perform additional studies beyond Cassini but not as } \\
\text { many as TSS. }\end{array}$ \\
\hline MMRSS & 4 & $\begin{array}{l}\text { Has the potential to perform additional studies beyond Cassini but not as } \\
\text { many as TSS. }\end{array}$ \\
\hline
\end{tabular}


Table 23. Complexity Scoring.

\begin{tabular}{|l|c|l|}
\hline Architecture & Score & Reason \\
\hline TSS & 1 & $\begin{array}{l}\text { Largest number of spacecraft. Complex operations and difficult tracking and } \\
\text { navigation. Complex design of Mother telecommunication system to relay } \\
\text { data from 40+ spacecraft. }\end{array}$ \\
\hline TS & 4 & $\begin{array}{l}\text { Simple mother design due to lack of instruments but more complex } \\
\text { operations and telecommunication design than RSS because of larger number } \\
\text { of CubeSats. }\end{array}$ \\
\hline RSS & 4 & $\begin{array}{l}\text { Simple operations and telecommunication system but more complex Mother } \\
\text { design to host instruments. }\end{array}$ \\
\hline MTS & 3 & $\begin{array}{l}\text { Complex operations due to two separate swarms. Complex } \\
\text { telecommunication system to relay data and perform tracking and navigation. }\end{array}$ \\
\hline RSTS & 3 & $\begin{array}{l}\text { Complex operations due to two separate swarms. Complex } \\
\text { telecommunication system to relay data and perform tracking and navigation. }\end{array}$ \\
\hline MMTS & 2 & $\begin{array}{l}\text { Complex design of two separate mothers that would need to stack on one } \\
\text { another. Operations of two individual swarms and large total number of } \\
\text { spacecraft. }\end{array}$ \\
\hline MMRSS & 2 & $\begin{array}{l}\text { Complex design of two separate mothers that would need to stack on one } \\
\text { another. Operations of two individual swarms and large total number of } \\
\text { spacecraft. }\end{array}$ \\
\hline
\end{tabular}

Table 24. Reliability Scoring.

\begin{tabular}{|l|c|l|}
\hline Architecture & Score & Reason \\
\hline TSS & 3 & $\begin{array}{l}\text { Large number of similar CubeSats that could replace one another if a failure } \\
\text { were to occur. However, only one Mother exists so if it failed the whole } \\
\text { architecture fails. }\end{array}$ \\
\hline TS & 1 & $\begin{array}{l}\text { If any spacecraft were to fail, no replacement exists. Large number of } \\
\text { CubeSats increases probability of a failure. }\end{array}$ \\
\hline RSS & 2 & $\begin{array}{l}\text { If any spacecraft were to fail, no replacement exists. Smaller number of } \\
\text { CubeSats than TS reducing probability of failure. }\end{array}$ \\
\hline MTS & 3 & $\begin{array}{l}\text { Every instrument has a potential replacement should it fail. However, only } \\
\text { one Mother exists. }\end{array}$ \\
\hline RSTS & 3 & $\begin{array}{l}\text { Every instrument has a potential replacement should it fail. However, only } \\
\text { one Mother exists. }\end{array}$ \\
\hline MMTS & 4 & $\begin{array}{l}\text { Two of every spacecraft exists so if a spacecraft were to fail, a second exists } \\
\text { that could replace. However, the large number of spacecraft increases the } \\
\text { potential of a failure. }\end{array}$ \\
\hline MMRSS & 5 & $\begin{array}{l}\text { With two near identical swarms and Mothers, if any one spacecraft were to } \\
\text { fail, a second exists that could replace it. }\end{array}$ \\
\hline
\end{tabular}


Table 25. Architecture Trade Study Summary.

\begin{tabular}{|l|l|l|l|l|l|l|l|l|}
\hline & Weight & TSS & TS & RSS & MTS & RSTS & MMTS & MMRSS \\
\hline Cost & $\mathbf{4 0 \%}$ & 1 & 4 & $\mathbf{5}$ & 2 & 4 & 1 & 1 \\
\hline Science Return & $\mathbf{3 0 \%}$ & 5 & 3 & $\mathbf{3}$ & 4 & 4 & 4 & 4 \\
\hline Complexity & $\mathbf{2 0 \%}$ & 1 & 4 & $\mathbf{4}$ & 3 & 3 & 2 & 2 \\
\hline Reliability & $\mathbf{1 0} \%$ & 3 & 1 & $\mathbf{2}$ & 3 & 3 & 4 & 5 \\
\hline & & & & & & & & \\
\hline Score & & 2.4 & 3.4 & $\mathbf{3 . 9}$ & 2.9 & 3.7 & 2.4 & 2.5 \\
\hline
\end{tabular}

The Remote Sensing Swarm was found to be the best architecture with a score of 3.9 based on the selection criteria. A close second was the RSTS. In order for the RSTS to win, Science Return and Reliability would need to be weighted more.

The Remote Sensing Swarm was given a 5 for Cost because it had the cheapest overall cost. Table 26 shows the cost relation between the different architecture. While the numbers could fluctuate, the general trend should hold. A score of 3 was given for Science Return because even though it would meet all the science goals of the Cassini mission, it didn't have the potential to enhance scientific discovery like the Target Specific Swarm did. The Remote Sensing Swarm scored a 4 for complexity because even though it had the fewest CubeSats, it added some complexity to the design of the Mother. Only a 2 was given for Reliability because of the increased potential for failure by having five separate spacecraft.

Table 26. Cost Relation Between Architectures.

\begin{tabular}{|l|l|}
\hline Architecture & Cost Compared to RSS \\
\hline RSS & $+\$ 0$ \\
\hline TS & $+\$ 100$ million \\
\hline RSTS & $+\$ 100$ million \\
\hline MTS & $+\$ 250$ million \\
\hline MMRSS & $>\$ 300$ million \\
\hline MMTS & $>\$ 300$ million \\
\hline TSS & $+\$ 1$ billion \\
\hline
\end{tabular}


With the Remote Sensing Swarm selected as the ideal architecture for replicating the Cassini Mission, further work was done to assess its technical feasibility. The architecture trade study was performed at a very high level assuming each architecture was feasible. While several CubeSats are slated to launch on the upcoming Exploration Mission 1 going to the moon [49] and the Insight mission to Mars [3], no CubeSat has yet flown in interplanetary space, let alone at Saturn. An initial bus design is proposed that could accommodate the remote sensing instruments as well as a conceptual Mother spacecraft to show their technical feasibility.

Because the instruments do not exist in a CubeSat form factor, a single bus was designed that could accommodate any of the remote sensing instruments and serve as a baseline for further detailed design. The proposed bus would offer the instruments $7 \mathrm{~W}$ of power and $6 \mathrm{U}$ out of a $12 \mathrm{U}$ CubeSat. It is important to note that even if a different architecture was chosen, the remote sensing CubeSat bus wouldn't change other than the propulsion system potentially being able to reduce propellant mass.

Detailed design could discover challenges but the detailed design of the spacecraft is not within the scope of this thesis. The proposed designs suggest that the architecture is technically feasible using current technologies with minimal modification.

\subsubsection{CubeSat Payloads}

To replicate the ISS measurements, one to two Intellicams with different optics could be used. The Intellicam is being developed by JPL as a low cost deep space imager based on Malin Space Science Systems ECAM-C50. The Intellicam will have an approximate power consumption of $2.5 \mathrm{~W}$ based on the heritage ECAM-C50 [50]. Intellicam will have 20 Mpixels and will fit in approximately $1.5 \mathrm{U}$. To replicate the abilities of the ISS, a filter wheel assembly would need to be developed and the spectral range expanded slightly. The total CubeSat instrument can be assumed to have approximately $5 \mathrm{~W}$ power consumption.

No CubeSat size hyperspectral instrument like VIMS currently has a comparable range. Existing products cover the visible spectrum of VIMS' range but not the IR components. Vims will likely prove one of the harder instruments to miniaturize simply because of the breadth of it spectral range. That said, detector technology has come a long way since Cassini with detectors now in the multiple Mpixel range making the 
challenge of replicating VIMS an optical one. While it will be difficult to miniaturize VIMS, it is assumed to be possible to create a hyperspectral imager capable of fitting into a $12 \mathrm{U}$.

UVIS could be replicated taking design of the R-Alice ultraviolet instrument and shrinking it to CubeSat size. R-Alice is $12 \times 20 \times 41 \mathrm{~cm}$ and would need to be shrank down to at least $10 \times 20 \times 20 \mathrm{~cm}$ to fit into a $12 \mathrm{U}$ CubeSat. R-Alice is already pretty power-efficient consuming just $4.0 \mathrm{~W}$ but any reduction is a good thing. R-Alice also has a similar spectral range as UVIS of $70-205 \mathrm{~nm}$ and would likely take minimal modifications to change the range to UVIS's $56-191 \mathrm{~nm}$. Because of UV light having small wavelengths and the already small size of heritage components, an UV spectrograph appears readily feasible to miniaturize to CubeSat size.

CIRAS will likely be another instrument that is hard to miniaturize because of the long wavelength of light it observes and its immense spectral range. CIRAS was a Michelson interferometer and the CubeSat version might need to be as well to achieve the range of $7-1000 \mu \mathrm{m}$.

The Birches IR spectrometer is an excellent example of the possibilities of miniaturization. Birches is a miniaturized version of the Ralph instrument that flew on New Horizons. Ralph was 49 x 40 x $29 \mathrm{~cm}$ with a mass of $11 \mathrm{~kg}$ and was successfully miniaturized into Birches with dimension $10 \mathrm{x} 10$ x $15 \mathrm{~cm}$, mass of just $2 \mathrm{~kg}$ and power consumption of $5 \mathrm{~W}$.

For this thesis, it is assumed that the Cassini instruments could be miniaturized to CubeSat form factors. This appears to be a valid assumption but achieving similar spectral ranges will be challenging with the smaller form factors. Another challenge with miniaturization is radiometric signal to noise ratios of the instruments. The Cassini remote sensing instruments generally had SNRs of 100. Even with high modern performance components, miniaturized CubeSat instruments will need longer integration times to achieve similar SNR. Having longer integration times will require the attitude control system to maintain stable pointing for longer periods of time adding complexity to the ADCS. Longer integration times will also reduce the total number of images taken unless Cassini had ample time between images.

One of the greatest benefits of moving the remote Sensing instruments to CubeSats is they would all be independent of each other. On Cassini, because they all shared a common boresight, the different 
instruments would have to share the 12 hour operation time with each other so if two instruments wanted to image two different targets, one would be forced to collect its data quickly so the other could have its turn. With the CubeSats, each one can point to wherever it likes whenever it likes, simplifying operations and coordination between the science teams. Independently pointing instruments will also decrease the time spent slewing between targets potentially compensating for the longer integration times required to achieve desired SNRs.

For this thesis, it will be assumed that all the instruments can miniaturized with the caveat that the exact same performance might not be possible. It is also assumed that the instruments could achieve power consumptions of approximately $5 \mathrm{~W}$.

\subsubsection{ADCS}

The default attitude and determination control system for high performance CubeSats is the XACT module made by Blue Canyon Technologies. The XACT system offers best in class pointing accuracy of $\pm 0.003^{\circ}$

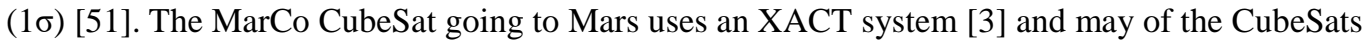
launching on Exploration Mission 1 use it as well. XACT has a modest operating power consumption of $2.5 \mathrm{~W}$ and a low power standby consumption of just $0.03 \mathrm{~W}$. XACT has a larger version with bigger reaction wheels called the XACT 50 meant for heavier 6 and 12U CubeSats while maintaining similar power consumptions. The XACT 50, shown in Figure 30, has a volume of $0.75 \mathrm{U}$ is baselined for all the CubeSats in the Remote Sensing Swarm.

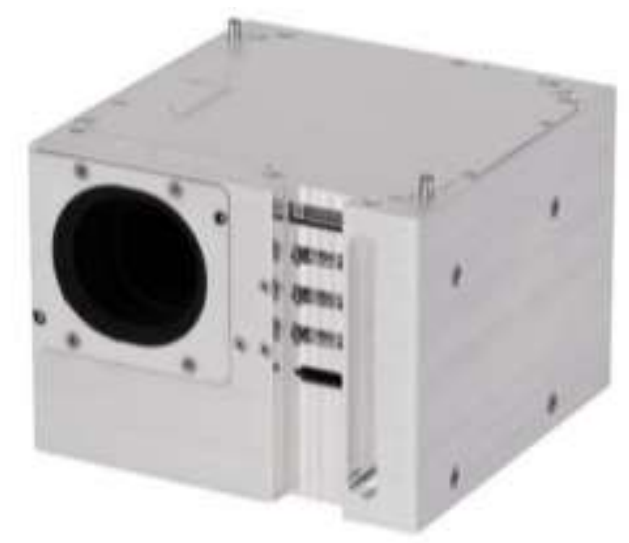

Figure 30. XACT 50 ADCS. Credit Blue Canyon Technologies. 


\subsubsection{Flight Computer}

For a flight computer, JPL's Leon 3 Sphinx CubeSat processor was baselined. JPL developed the processor for the NEA Scout and Lunar Flashlight missions as a reliable and radiation hardened flight processor for interplanetary missions. The processor consumes between 1.5 and $7 \mathrm{~W}$ during nominal operations [52]. For the sake of analysis, it is assumed the Sphinx processor consumes an average of $2.5 \mathrm{~W}$ and is capable of a low power standby mode with a consumption of $0.5 \mathrm{~W}$. Other CubeSat flight processors exist, notably the Tyvak/Cal Poly Intrepid board, that have lower power consumptions and even lower standby modes. However, these processors aren't explicitly designed for deep space so for an initial design they weren't considered. It is easier to reduce power consumption than increase it in further studies. The Sphinx processor is shown in Figure 31.

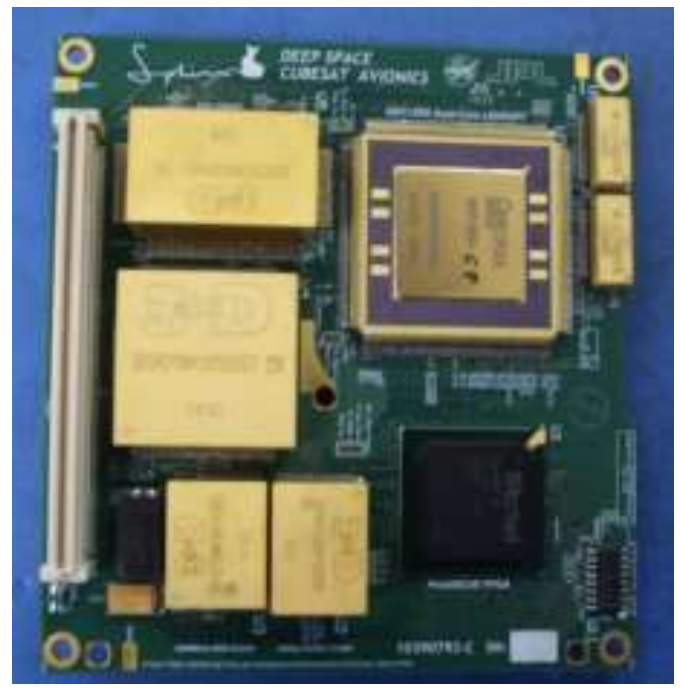

Figure 31. Leon 3 Sphinx Computer. Credit Jet Propulsion Laboratory.

\subsubsection{Propulsion}

The CubeSats will need their own propulsion to perform trajectory correction throughout the four year tour. Cassini allocated $497 \mathrm{~m} / \mathrm{s}$ of delta V for trajectory corrections so the CubeSats will need to perform this much as well. A change of velocity of $500 \mathrm{~m} / \mathrm{s}$ is extremely difficult for a CubeSat to accomplish and is impossible with traditional cold gas CubeSat systems. Electric propulsion capable of $\sim 2 \mathrm{~km} / \mathrm{s}$ of delta $\mathrm{V}$ 
[53] is becoming available but the power required to operate is too high for the low power production environment at Saturn that receives about $1 \%$ of the solar energy as Earth. But recent advancements in monopropellant systems bring high delta $\mathrm{V}$, high thrust trajectories into the realm of possibility. Because the CubeSats and Mother follow the Cassini trajectory, the CubeSats will need a high thrust system like what monopropellant systems can provide. Aerojet Rocketdyne is in the process of producing a propulsion system that has a total system impulse of 3,360 N-s [54]. Simply dividing the total impulse by the mass of the spacecraft gives an approximate total delta V capability. Assuming the CubeSats are $12 \mathrm{~kg} 6 \mathrm{Us}$, the propulsion unit can provide approximately $280 \mathrm{~m} / \mathrm{s}$ of delta $\mathrm{V}$ which is simply not enough. A solution to this problem is to expand the CubeSats to $12 \mathrm{U}$ and fit two propulsion units in. Assuming the only increase in mass is $3.75 \mathrm{~kg}, 2.75 \mathrm{~kg}$ for the propulsion unit and $1 \mathrm{~kg}$ for extra structure, the new total system impulse of $6,720 \mathrm{~N}$-s can provide about $425 \mathrm{~m} / \mathrm{s}$ of delta V. While not the $497 \mathrm{~m} / \mathrm{s}$ allocated by Cassini, the propellant tanks could be expanded. The $497 \mathrm{~m} / \mathrm{s}$ allocation was also created before Cassini launched for trajectory corrections. Actual delta V for the mission was likely lower so the proposed propulsion system could be sufficient without modification. It should be noted that the CubeSats could position themselves closer to Titan to gain a greater gravity assist but doing so would not alleviate delta V concerns as most of Cassini's delta V allocation was for post flyby trajectory correction, not orbital maneuvering.

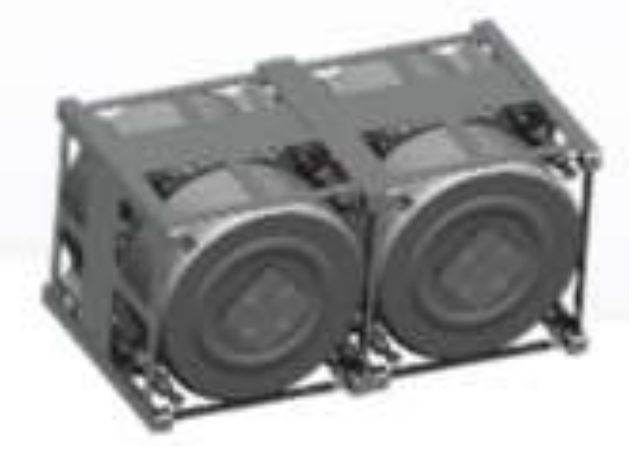

Figure 32. Aerojet Rocketdyne CubeSat Propulsion System.

\subsubsection{Communication}

Current NASA missions still use $\mathrm{X}$ band as their primary communication band; Cassini used it [14] and Juno is currently using it [55]. It is assumed for the sake of analysis that the Mother will also use X band. Since the Mother will use X band, it is desirable for the CubeSats to also use X band to utilize the Mother's 
existing systems and not require it to have a separate system for communicating with the CubeSats. Using $\mathrm{X}$ band keeps system design simple and has the added benefit of JPL already having a deep space transponder. The Iris transponder, shown in Figure 33, uses X band and is purpose built for deep space communication. Iris also has the built-in capability for two way ranging and can support Doppler tracking. Simply modifying the Electra radio to use $\mathrm{X}$ band and using it in conjunction with Iris could be an elegant solution for the challenge of tracking and navigating the CubeSats. Iris consumes $26 \mathrm{~W}$ in full transmit/receive mode but outputs $5 \mathrm{~W}$ RF power [56].

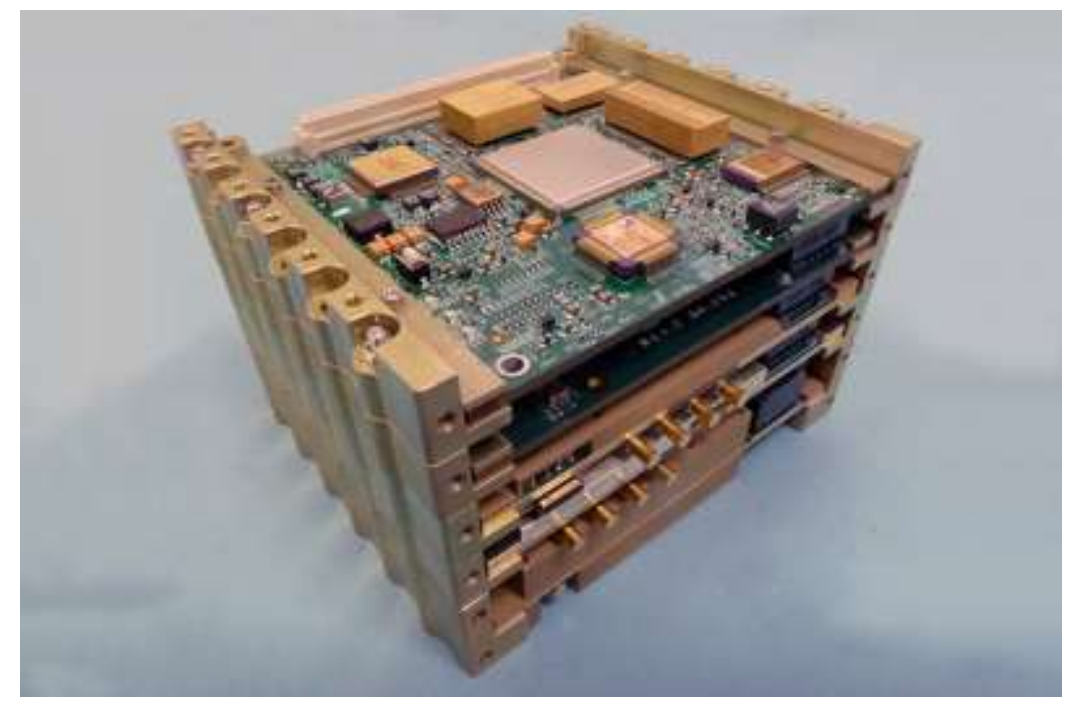

Figure 33. Iris Deep Space Transponder. Credit Jet Propulsion Laboratory.

If the CubeSats use a reflect array antenna design like the MarCo CubeSat, emergency direct to Earth communication is possible. The reflect array antenna has a gain of $28 \mathrm{dBi}$ [56] that could enable a bit rate as high as $16 \mathrm{bps}$.

The link budget for direct to Earth communications is shown in Table 27 for the Iris radio using a Marco reflect array antenna transmitting to the DSN $70 \mathrm{~m}$ dish. 
Table 27. CubeSat Direct to Earth Link Budget.

\begin{tabular}{|l|l|l|}
\hline \multicolumn{3}{|c|}{ X band Downlink } \\
\hline Bit rate & 0.016 & $\mathrm{Kbps}$ \\
\hline Frequency & 8.45 & $\mathrm{GHz}$ \\
\hline Slant range & $1.5 \mathrm{E}+09$ & $\mathrm{~km}$ \\
\hline \multicolumn{3}{|c|}{ Environment } \\
Wave Length & 0.035 & \\
\hline Space loss & 294.66 & $\mathrm{~dB}$ \\
\hline polarization & 3 & $\mathrm{~dB}$ \\
loss & & \\
\hline \multicolumn{2}{|c|}{ S/C } \\
\hline RF power & 5 & $\mathrm{~W}$ \\
\hline Line losses & 1 & $\mathrm{~dB}$ \\
\hline Antenna Gain & 28 & $\mathrm{~dB}$ \\
\hline Pointing loss & 1 & $\mathrm{~dB}$ \\
\hline Noise Temp & 0 & $\mathrm{~dB}$ \\
\hline \multicolumn{2}{|c|}{ Receiver } & \\
\hline Antenna Gain & 74.6 & $\mathrm{~dB}$ \\
\hline Pointing Loss & 0.1 & $\mathrm{~dB}$ \\
\hline Noise Temp & 13.1 & $\mathrm{~dB}$ \\
\hline Modulation & 2.75 & $\mathrm{~dB}$ \\
Loss & & \\
\hline Required & 4 & $\mathrm{~dB}$ \\
Power & & $\mathrm{dB}$ \\
\hline EB/n0 & 10.53 & $\mathrm{~dB}$ \\
\hline Margin & 6.53 & \\
\hline
\end{tabular}

However, the ability to both communicate with Earth and the Mother would require an expansion of Iris's frequency range to both transmit and receive over between 7 and $8.5 \mathrm{GHz}$. The expansion is necessary because the DSN only transmits between $7.1-7.3 \mathrm{GHz}$ and receives between 8.4 and 8.5 which means the Mother receives and transmits in the reverse frequencies. For the CubeSat to communicate with both the Mother and Earth, Iris needs to be able to transmit and receive in both frequency ranges. At 16 bps, direct to Earth communication would only be used for emergency operations and trouble shooting. 
Flying in formation only as far as $1 \mathrm{~km}$ from the Mother allows the CubeSat to have extremely high data rate communication with the Mother. Table 28 shows the CubeSat to Mother relay data rate to be as high as 1 Gbps conservatively. This data rate is the CubeSat transmitting from a single $\mathrm{X}$ band patch antenna with $5 \mathrm{dBi}$ of gain [56] to the Mother's low gain antenna. The reason the CubeSat would communicate with the Mothers low gain antennas is because they are omni directional and so don't require the Mother to have to point toward the CubeSat. The CubeSat can be in any direction in the Mother's coordinate frame and still achieve 1 Gbps. The $18.5 \mathrm{~dB}$ pointing loss for the Mother conservatively accounts for the worst case of the CubeSat being exactly $90^{\circ}$ off boresight of the low gain antenna [55].

Table 28. CubeSat to Mother Relay Link Budget.

\begin{tabular}{|l|c|c|}
\hline \multicolumn{3}{|c|}{ Inter-Satellite Link } \\
\hline Bit rate & 1000000 & $\mathrm{Kbps}$ \\
\hline Frequency & 7.2 & $\mathrm{GHz}$ \\
\hline Slant range & 1 & $\mathrm{~km}$ \\
\hline Environment & \\
\hline Wave Length & 0.041638 & \\
\hline Space loss & 109.5944 & $\mathrm{~dB}$ \\
\hline polarization loss & 3 & $\mathrm{~dB}$ \\
\hline Atmospheric loss & 0 & $\mathrm{~dB}$ \\
\hline \multicolumn{1}{|c|}{ S/C } & & \\
\hline RF power & 5 & $\mathrm{~W}$ \\
\hline Line losses & 1 & $\mathrm{~dB}$ \\
\hline Antenna Gain & 5 & $\mathrm{~dB}$ \\
\hline Pointing loss & 0.5 & $\mathrm{~dB}$ \\
\hline Noise Temp & 0 & $\mathrm{~dB}$ \\
\hline Receiver & & \\
\hline Antenna Gain & 8 & $\mathrm{~dB}$ \\
\hline Pointing Loss & 18.5 & $\mathrm{~dB}$ \\
\hline Line Loss & 8 & $\mathrm{~dB}$ \\
\hline Noise Temp & 0 & $\mathrm{~dB}$ \\
\hline Modulation & 2.75 & $\mathrm{~dB}$ \\
\hline Rosses & & \\
\hline EB/n0 & & \\
\hline Margin & & $\mathrm{dB}$ \\
\hline
\end{tabular}


With relay data rates of at least $1 \mathrm{Gbps}$, the CubeSat will hardly need to spend any time in communication with the Mother. Analyzing the Cassini data down link allocations for each instrument, the ISS was the highest data producer being allocated a maximum of $3 \mathrm{~Gb}$ per science period. With a data rate of $1 \mathrm{Gbps}$, the CubeSats will never need more than a few seconds to relay their science and engineering telemetry. The only reason a CubeSat would need to be in communication for much longer than a few seconds is for tracking and navigation. For these purposes, it is assumed that a CubeSat will be in communication with the Mother for 10 minutes once per science operation.

\subsubsection{Thermal}

The thermal environment will be challenging for the CubeSats as they will only receive $1 \%$ of the solar energy they would get at Earth. Because of the low solar energy, it was assumed that all the power required to keep the CubeSats within operating temperatures was produced by the CubeSats. Figure 34 shows the internal heating power required to keep the CubeSat internals at $-10,-20$ and $-30 \mathrm{C}$ for various emissivities

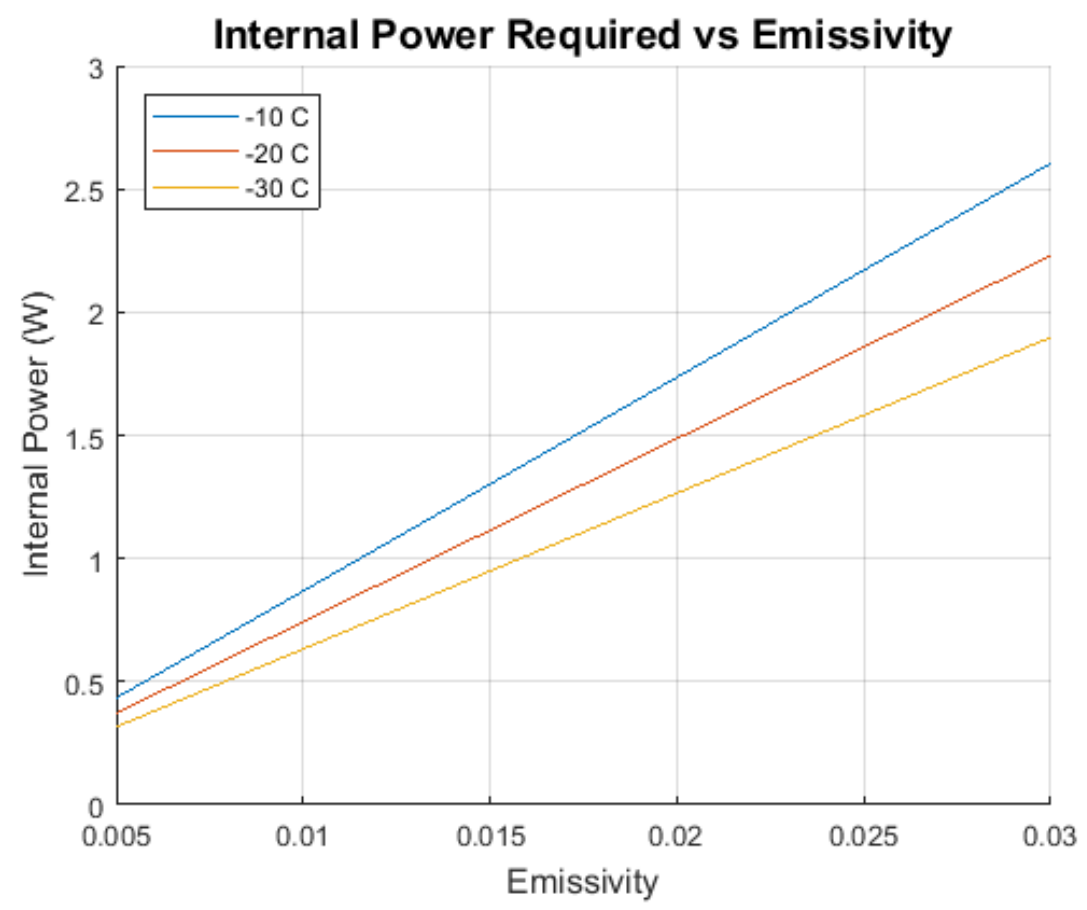

Figure 34. Heating Power Required vs Emissivity.

Figure 35 shows the number of layers of multi-layer insulation (MLI) needed to achieve the emissivities given in Figure 34. While the lowest possible heater power is desired, the number of layers of MLI needs to 
be reasonable. For the CubeSats, MLI with 12 layers was chosen since 12 layers corresponds to an emissivity of about 0.013 requiring $1 \mathrm{~W}$ of input power to maintain the internal temperature of the CubeSat at -20C. The low end of operating temperatures for many commercial off the shelf CubeSat components is -20C leading to it being chosen as the desired maintenance temperature. While more layers would reduce the amount of power required, $1 \mathrm{~W}$ of heater power is conservative and 12 layers seems perfectly reasonable for a CubeSat. The CubeSats will likely be able to maintain higher temperatures due to operating components producing heat.

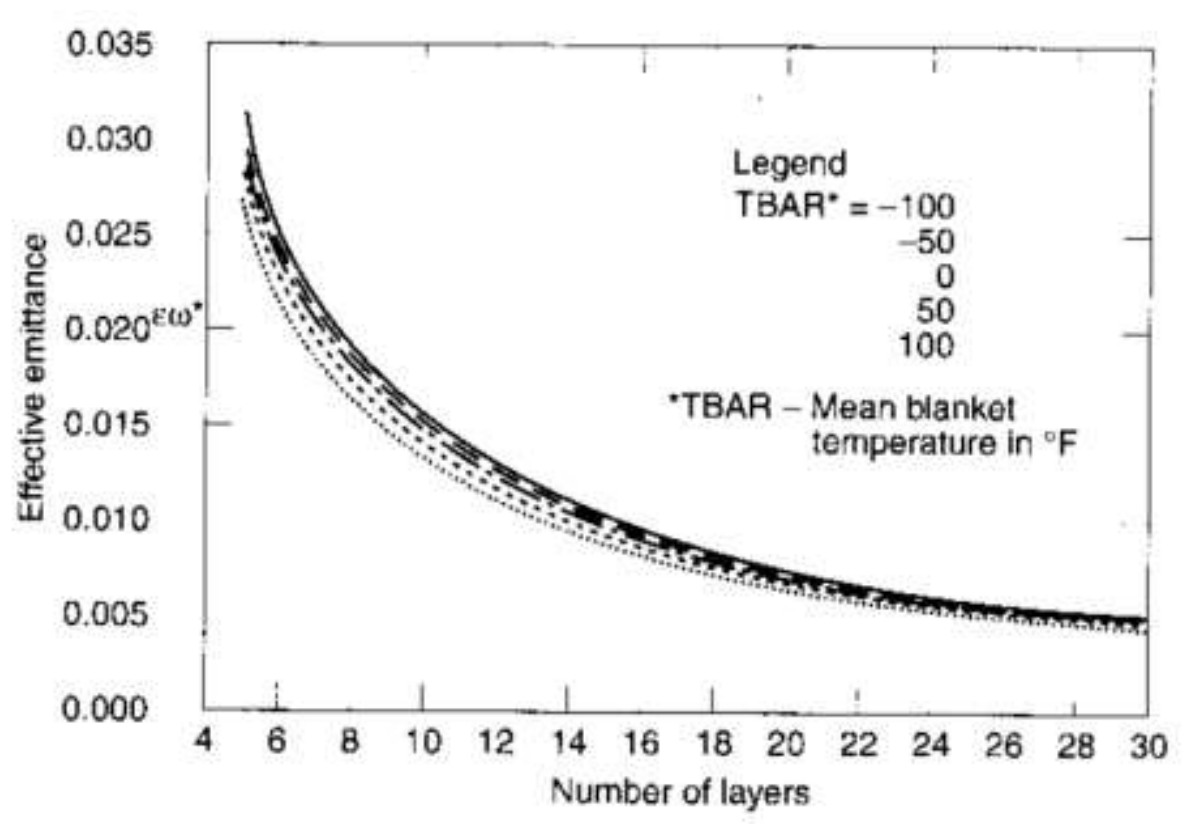

Figure 35. Emissivity vs Number of Layers of MLI [57].

\subsubsection{Power}

Power generation is the greatest challenge for CubeSats at Saturn and for deep space in general. There is a concept design for a CubeSat RTG but it's output is only $50 \mathrm{~mW}$ [58] which is simply not enough power. Due to the prevalence of existing solar panel systems for CubeSats, only solar power was considered for the Remote Sensing Swarm CubeSats.

Saturn's semi major axis is $9.582 \mathrm{AU}$ [47], so the available solar energy is $1.09 \%$ of the available energy at Earth. The current largest solar panels for CubeSats produces 120W at Earth [53] so if the same solar panels were used at Saturn they would produce just 1.31 W. Lunar IceCube used two arrays of three panels 
that are $6 \mathrm{U}, 0.3 \times 0.2 \mathrm{~m}$, each as shown in Figure 36. The flight computer alone consumes more power than would be generated during normal operations demanding alternative be investigated.

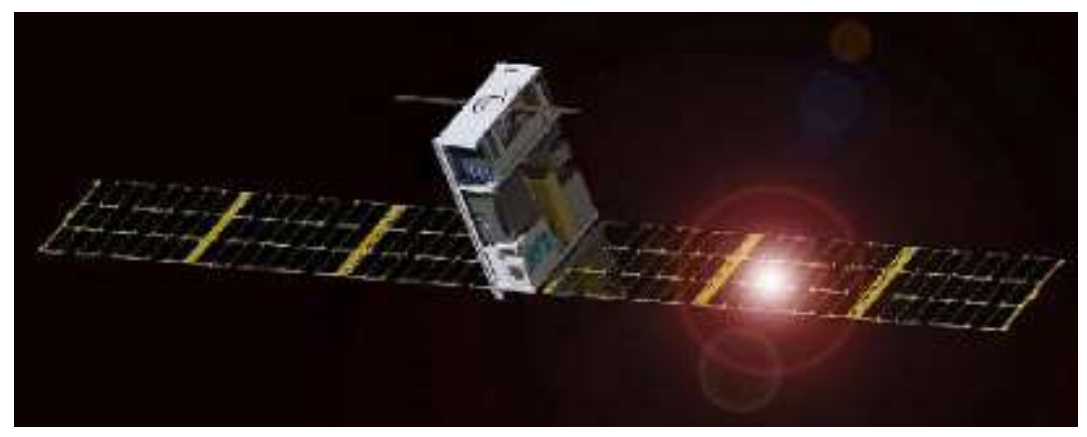

Figure 36. Lunar IceCube. Credit Morehead State University.

If a similar 6 panel architecture used the previously mentioned advanced LILT solar cells with $36 \%$ efficiency and $90 \%$ of the panel area is covered by the cells, $1.74 \mathrm{~W}$ could be generated. The increase in power production is beneficial, but more power is desired. Expanded arrays containing eight and ten cells could produce 2.33 and $2.91 \mathrm{~W}$ respectively. If a $12 \mathrm{U}$ CubeSat was used as suggested by the propulsion analysis, four strings of 5 panels would be possible and produce $5.82 \mathrm{~W}$. Even with 20 panels, barely enough power is generated to operate the flight computer and the ADCS let alone an actual payload.

Having much more than 20 panels starts to become infeasible because the CubeSat deployer limits the volume the panels can occupy while stowed. Adding more panels will start to eat into the internal volume which is a problem as the payloads will likely require all the volume they can get. Potentially custom deployers could be built to expand the volume available for the stowed solar arrays but the extra length raises concern over the panel's dynamic stability when the CubeSats are slewing.

Cassini spent half of its time communicating with Earth and during that time, did not collect images with the remote sensing instruments. For the CubeSats, this means that half of the time could be spent charging batteries to supplement data collecting periods.

However, during imaging operations, it cannot be assumed that the solar panels will be pointed at the sun. For this reason, it was assumed that no power is produced during imaging operations. While conservative, this assumption provides a good baseline. Figure 37 shows how long it would nominally take to recharge 
batteries versus how much power was consumed for each of the solar array architectures. While recharging, it is assumed that the solar panels are perpendicular to the sun, $1 \mathrm{~W}$ of heat is being generated the flight computer is in a low power sleep mode drawing only $0.5 \mathrm{~W}$ and all other systems are shut down. It is also assumed that the power distribution system is $90 \%$ efficient.

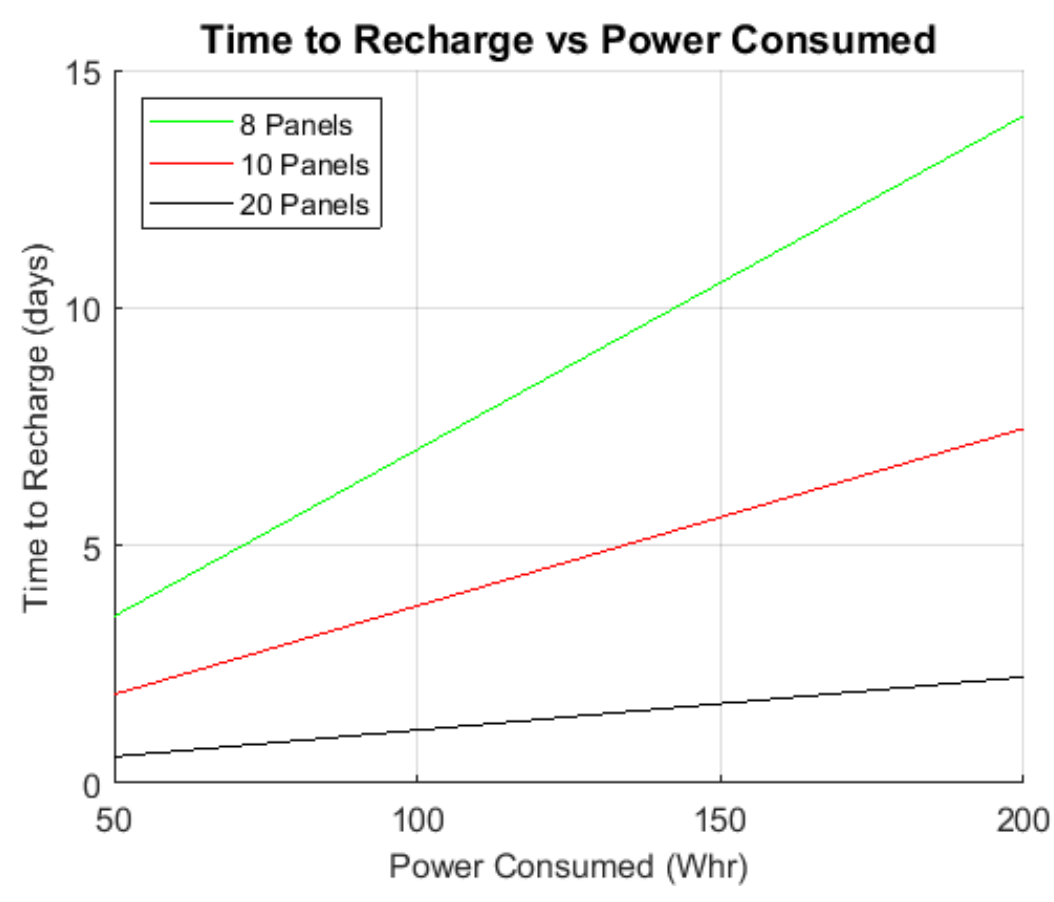

Figure 37. Time to Recharge Batteries vs Power Consumed.

For the CubeSat to maintain Cassini's 12 hour operation rate, it would need to fully recharge in 12 hours after operating for 12 hours. Even with 20 panels, the CubeSat can only consume less than $50 \mathrm{Whr}$, or 4.17 $\mathrm{W}$ an hour, and still recharge in 12 hours. This leaves no power for the payload if the flight processor and ADCS run at nominal power. More time will therefore be needed to recharge and generate more power. Extending the recharge time will either cut into mission operations or force the mission duration to be extended. An extension of the recharge time to 24 hours would provide $90 \mathrm{Whr}$ of charge. However, recharging for 24 hours would either reduce imaging operations by $33 \%$ or extend the mission by two years. Accounting for $12 \mathrm{Whr}$ for heating, $4 \mathrm{Whr}$ for 10 minutes of communications, and an average of 1 Whr for the propulsion system and the power distribution systems efficiency of $90 \%$, only $64 \mathrm{~W}$ are available for the ADCS, flight computer and payload. With the ADCS and flight computer together 
consuming $60 \mathrm{Whr}, 4 \mathrm{Whr}$ are left for the payload. With an available capacity of $4 \mathrm{Whr}$ to draw from, the payload can have a power consumption of only $0.33 \mathrm{~W}$. A further extension of the recharge time to 48 hours would allow for a capacity of $179 \mathrm{Whr}$ and enable the payload to have an operational power of $7.0 \mathrm{~W}$ at the cost of increasing the mission duration by 6 years or reducing the imaging operation period by $60 \%$.

Both the Birches and Intellicam instruments consumed just $5 \mathrm{~W}$, so having $7 \mathrm{~W}$ available allows for margin in the Remote Sensing Swarm CubeSats power consumption. However, losing $60 \%$ of the operation time, and therefore $60 \%$ of the remote sensing data is highly undesirable. Extending the mission duration by six years is also highly undesirable because it will add as much as $\$ 624$ million in operation costs and will increase complexity due to reliability concerns. A solution to this is to add duplicate remote sensing CubeSats and space out their operation such that 9.6 hours or $80 \%$ of imaging can be achieved every day. Adding more CubeSats to make up the remaining 2.4 hours increases the risk of needing a larger launch vehicle so wasn't considered but could be an option in further studies. Adding four CubeSats would only conservatively add \$120 million in cost accounting for savings in CubeSat manufacturing. Adding four CubeSats is much more cost effective than adding six years of mission operation and doesn't change the outcome of the trade study because the same power challenges would affect all the other architectures. Work should also be performed to reduce the power consumption of the computer and ADCS to reduce the time required to recharge.

The extra CubeSats have the added benefit of greatly increasing the reliability of the system. If one of the CubeSats were to fail, its duplicate could still fill its role, albeit, at a loss of data. Another added benefit is the potential for the same two instruments to operate simultaneously and image the same target from different locations creating a stereographic image, which was not possible with Cassini. Stereographic images would be especially beneficial with the ISS instrument as they would enable the creation of topographical maps of the moons surfaces. As Enceladus was found to have a liquid subsurface [59], there is a high interest in going back [60] and even placing a lander on the surface next to the cryovolcanic geysers that were discovered. Stereographic imaging would better help map the surface and locate an appropriate landing site for a future lander mission. Another thing simultaneous instrument operation would enable is imaging different location of the same target. For example, one instrument could image the 
equator of Saturn while the co-instrument images higher latitude allowing scientists to see how latitude effects an atmospheric phenomenon.

\subsubsection{Radiation}

Many deep space missions are concerned about radiation but because the CubeSats would stay in their radiation hardened deployers until reaching Saturn, the radiation hazard is greatly reduced. Saturn has a very benign radiation environment unlike Jupiter. Figure 38 shows the total accumulated dose of a notional four year Saturn probe mission for various aluminum shield thicknesses in mil, or thousandths of an inch. For aluminum shielding of just 10 mil which is only $0.254 \mathrm{~mm}$, a total dose of only $1 \mathrm{krad}$ is accumulated. Having shielding less than $0.5 \mathrm{~mm}$ thick on the CubeSats seems very feasible.

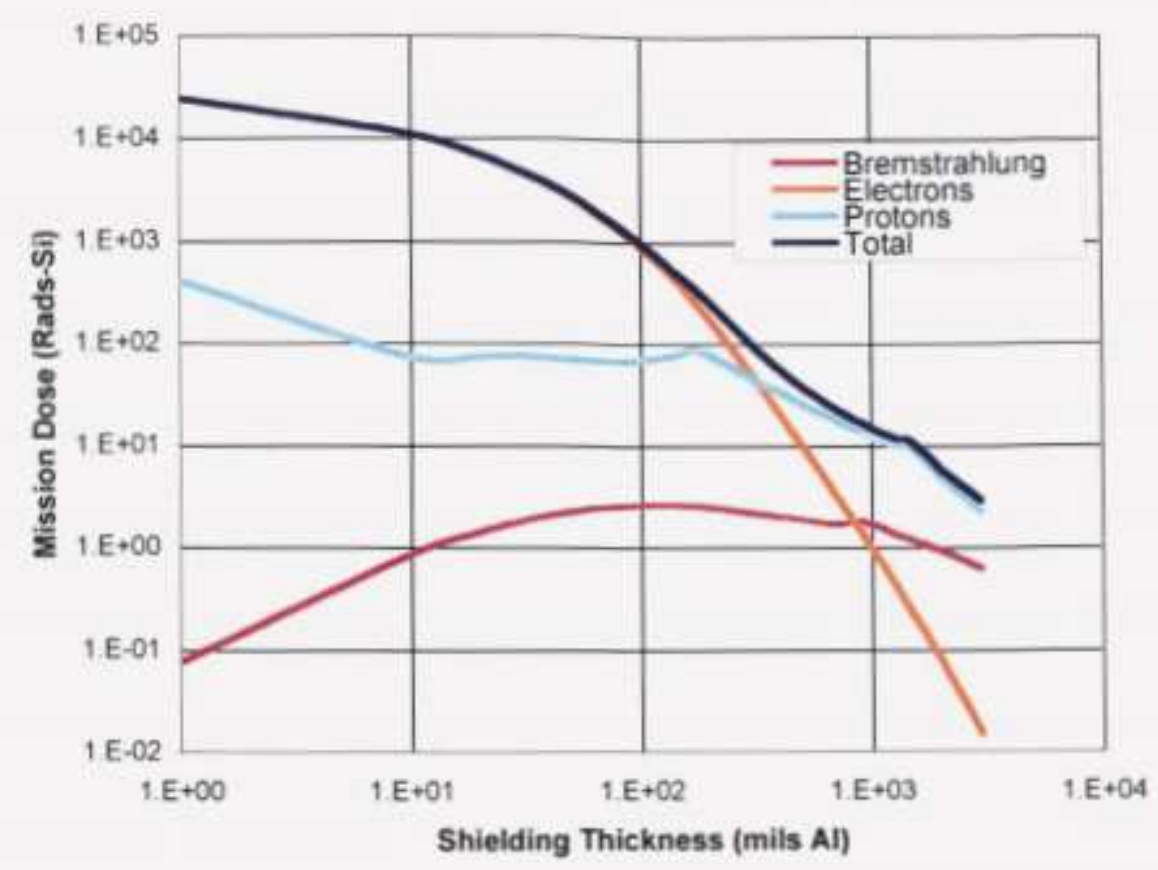

Figure 38. Total dose of the proposed Prometheus Saturn Moon Mission which had a four year minimum mission duration at Saturn [61] [62].

\subsubsection{CubeSat Design}

Because of the need for large propulsion tanks and 20 panel solar array, the Remote Sensing Swarm will consist of 8 12U CubeSats, four pairs of identical CubeSats to house modern miniaturized versions of the ISS, VIMS, CIRS and VIMS. The decision to go to a $12 \mathrm{U}$ package won't have any effect on the trade study 
because each architecture would require $12 \mathrm{U}$ CubeSats for the same reasons. With the $12 \mathrm{U}$ form factor, more volume is available for the instruments increasing their chance to be miniaturized and fit inside the CubeSat.

The 12U platform would allow for an instrument volume of almost $6 \mathrm{U}$. However, to meet propulsion requirements, the mass needs to be kept below $15.75 \mathrm{~kg}$ so mass would likely be the driving requirement, not volume. The same basic bus could be used for the eight CubeSats and is illustrated in figure. shows the dimensions and layout of the solar arrays. Two of the sides don't extend radially so they can provide a structure for the optically transparent reflect array [63].

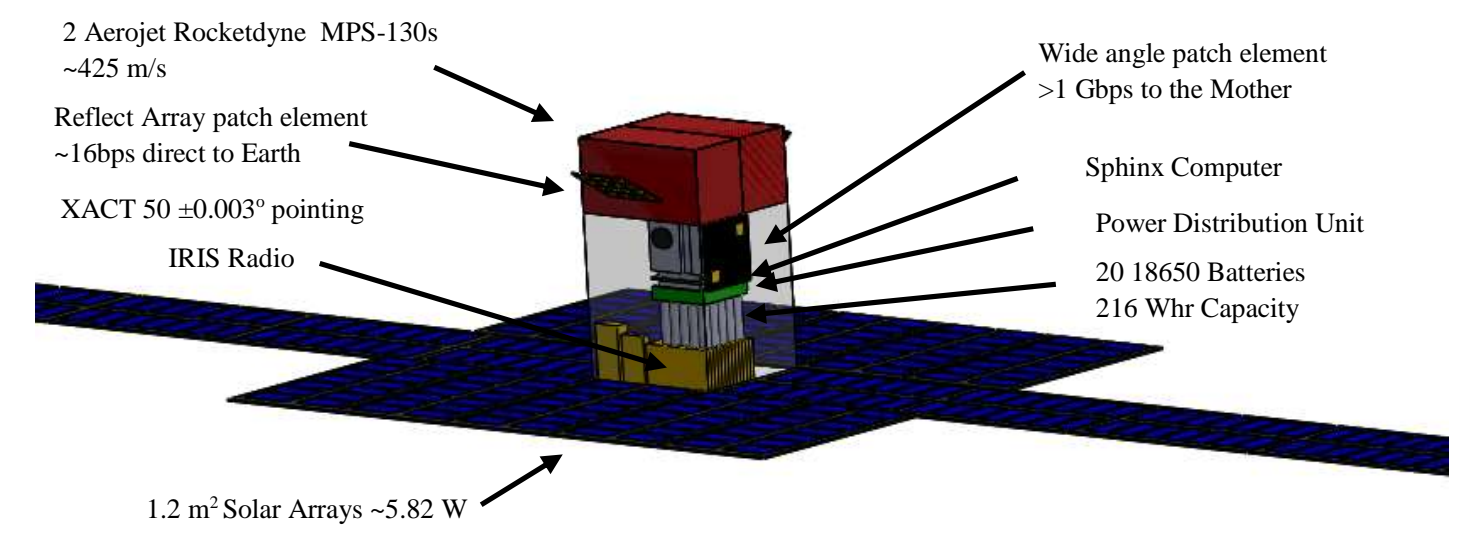

Figure 39. Remote Sensing CubeSat Bus.

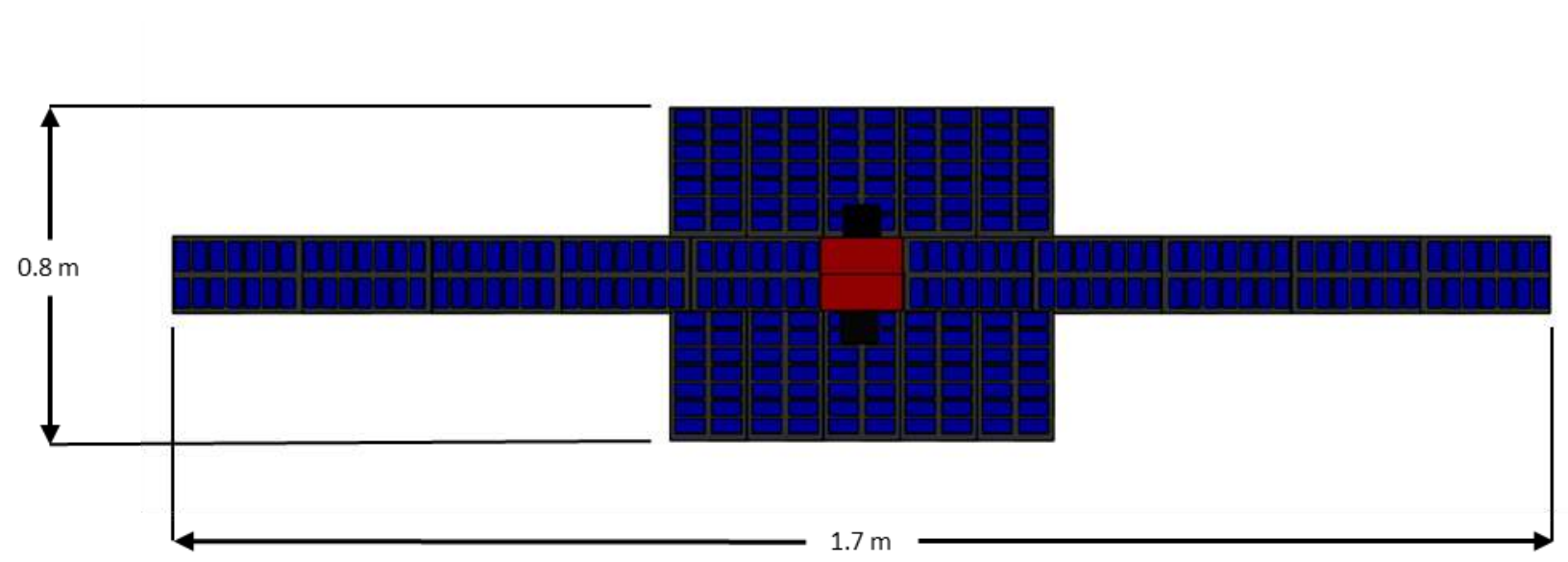

Figure 40. Remote Sensing CubeSat Solar Array Layout. 
Table 29. Remote Sensing CubeSat Bus Summary.

\begin{tabular}{|l|l|l|}
\hline & Mass & Power \\
\hline MPS 130 Thruster & $5.52 \mathrm{~kg}$ & $40 \mathrm{~W}$ \\
\hline XACT 50 ADCS & $1.23 \mathrm{~kg}$ & $2.5 \mathrm{~W}$ \\
\hline Sphinx Flight Computer & 0.2 & $2.5 \mathrm{~W}$ \\
\hline IRIS Radio & $1.2 \mathrm{~kg}$ & $26 \mathrm{~W}$ \\
\hline Batteries & $0.89 \mathrm{~kg}$ & $216 \mathrm{Whr}$ (Capacity) \\
\hline EPS & $\sim 0.2$ & $\mathrm{~N} / \mathrm{A}$ \\
\hline Thermal & $\sim 0$ & $1 \mathrm{~W}$ \\
\hline Structure & $\sim 1 \mathrm{~kg}$ & N/A \\
\hline Remote Sensing Payload & $6.5 \mathrm{~kg}$ & $7 \mathrm{~W}$ \\
\hline Total & $\mathbf{1 5 . 7 5}$ & $\begin{array}{l}\text { Operation: } \mathbf{1 2} \text { W } \\
\text { Sleep: 1.5 W }\end{array}$ \\
\hline
\end{tabular}

\subsubsection{Mother Design}

Juno is used as the baseline design for the Mother as it has similar propulsion capabilities and is the most distant solar powered spacecraft to date. This section discusses some of the modifications Juno would need to be used as the Mother for Remote Sensing Swarm architecture.

As was shown previously, a mother with similar solar panel area as the Juno spacecraft utilizing next generation 36\% efficient Low Incidence and Low Temperature solar cells [37] could generate as much power as $240 \mathrm{~W}$ at Saturn. Using new Roll Out Solar Array technology, which is 20\% lighter [38] than traditional solar arrays like the ones used on Juno, $290 \mathrm{~W}$ could be produced at Saturn for the same mass as Juno's solar arrays. Juno's three solar panels were $2.7 \times 9 \mathrm{~m}$ for a total surface area of $>60 \mathrm{~m}^{2}$ and an active power generating area of $45 \mathrm{~m}^{2}[30]$.

The only things the Mother needs to power are its own flight computer, star trackers, deep space transponder, telemetry control unit, ultra stable oscillator, $\mathrm{X}$ band traveling wave tube amplifier and the fields and particles instruments. 
Table 30. Mother Power Budget.

\begin{tabular}{|l|l|}
\hline Component & Power $(\mathbf{W})$ \\
\hline Computer & 25 \\
\hline Startrackers & 5 \\
\hline DST & 10.2 \\
\hline Telemetry Controller & 5.1 \\
\hline USO & 3.0 \\
\hline TWTA & 53.7 \\
\hline Fields and Particles Instruments & 84 \\
\hline Total & $\mathbf{1 8 6}$ \\
\hline
\end{tabular}

If the extended ROSA arrays are used, just over 100W are left over for thermal control of the Mother.

Further design and analysis is required to determine if this is sufficient but if it is not, simply increase the area of the solar arrays. One of the key benefits of ROSA array other than being lighter is that they can be lengthened to add area without having to add a whole extra panel like traditional solar array.

Extra batteries would have to be added to Juno to supply power during eclipse. The batteries will also double as being the power source for the RSS and Radar when they are on so power doesn't need to be taken from the fields and particles instruments. The driving case is when the RSS is on during eclipse with the avionics, instruments and thermal together consuming $376 \mathrm{~W}$. Baselining an eclipse at Titan distances, the mother will be in shadow for about five hour thus, the batteries must have a capacity of at least 1880 Whr. To meet the capacity requirements, the Mother would need ten of Juno's batteries [64]. To increase reliability and decrease the depth of discharge, 12 cells should be used. Increasing the number of cells from 2 to 12 would add $16.8 \mathrm{~kg}$.

Juno's high gain antenna would need to be replaced with a larger $4 \mathrm{~m}$ antenna to be used with the Cassini Radar and RSS. Increasing the diameter of the antenna will likely require the solar arrays to shift outward so they aren't shaded by the dish. While increasing the size of the dish adds some mass to the spacecraft, it is mitigated by that fact that Juno's titanium electronics vault won't be necessary. Much of the design work that went into Juno went towards making it radiation hard. While the Mother's journey to Saturn will be two years longer than Juno's, the radiation environment the Mother will experience at Saturn is incredibly benign compared to the environment found at Jupiter. 
Juno probe had eight instruments not including the Gravitational Wave experiment that used Juno's telemetry system. Those eight instruments had a total mass of $128.5 \mathrm{~kg}$ and power consumption of $64.5 \mathrm{~W}$ [65] [66] [67] [68] [69] [70] [71] [72].

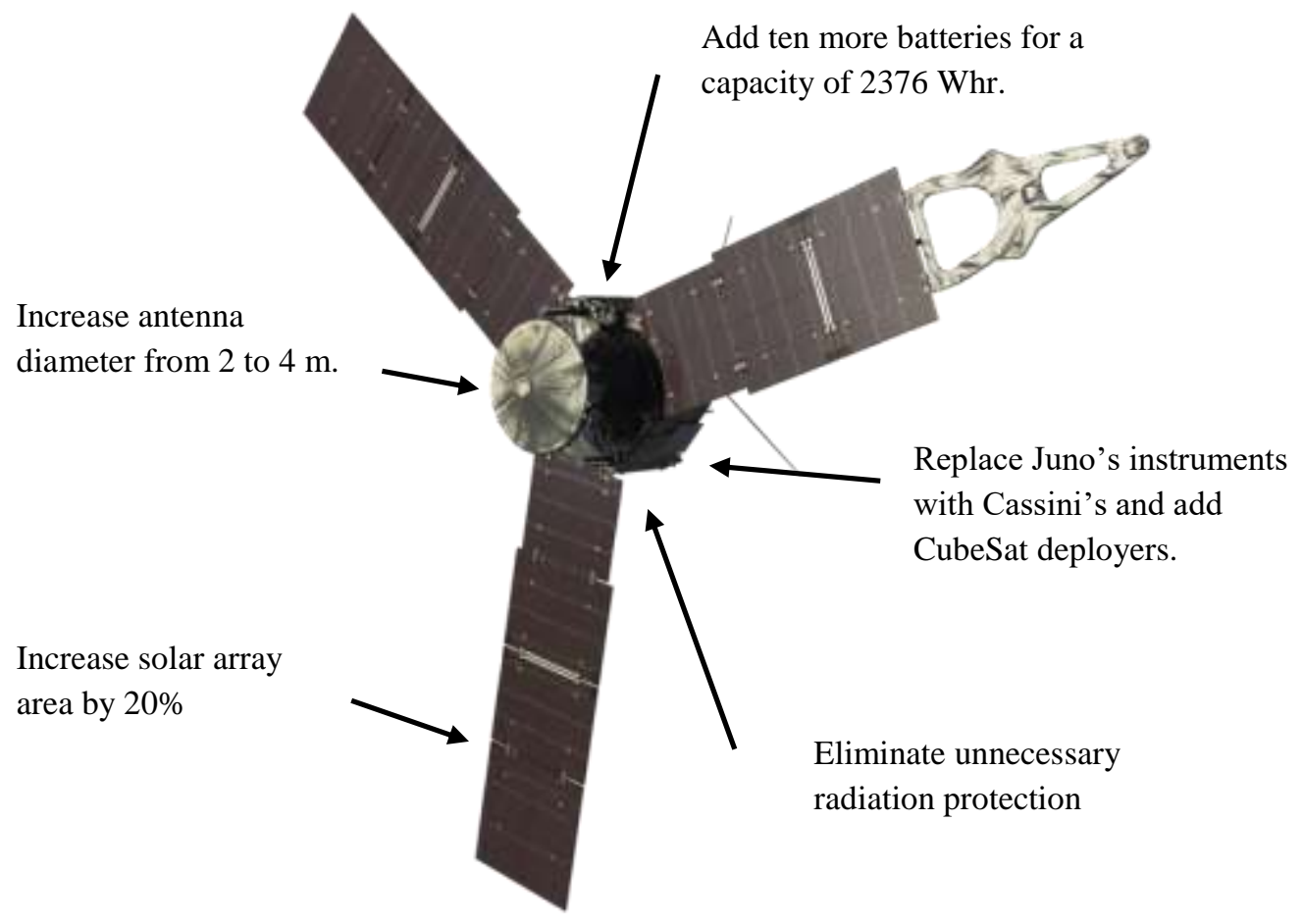

Figure 41. Juno and its Proposed Modifications for Motherhood.

\subsection{Summary}

The Cassini remote sensing pallet provided structurally and thermally stable platform to house all four remote sensing instruments in coalignment as shown in Figure 42. Having the boresights aligned allowed for tremendous collaboration between the instruments. However, being coaligned and hard mounted to Cassini drove the complexity of the Cassini spacecraft. 


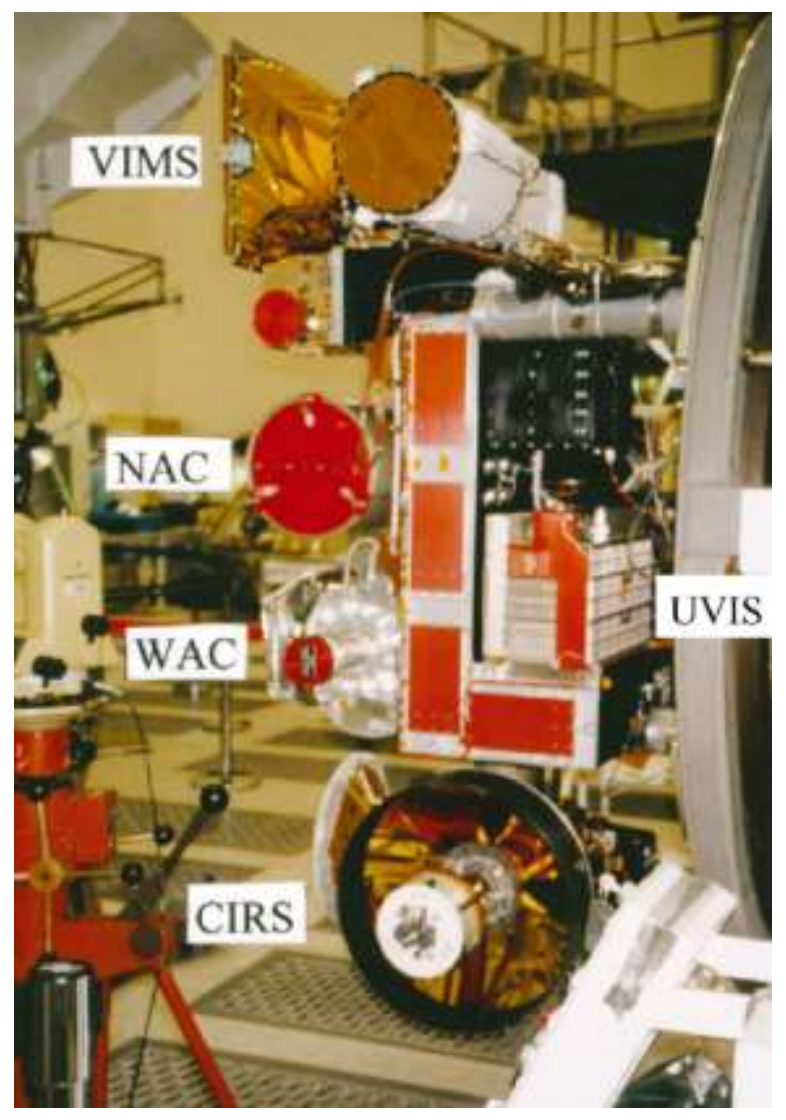

Figure 42. Remote Sensing Pallet.

The instruments worked together to meet the overarching Cassini mission goals of determining the foundation and history of the Saturn system. For some investigations, however, only one instrument needed to or was even capable of performing the desired measurement. Even worse was when instruments needed to image different targets. An example of this would be if the ISS wanted to image Titan during a flyby but UVIS, whose wavelengths can't penetrate Titans atmosphere, wanted to image the rings because of an occultation opportunity. The two instruments can't both perform their desired measurement due to their coaligned boresight.

Another problem that, while solvable, added tremendous complexity to the mission was when two targets were to be imaged in close temporal succession but were spatially far apart. The spacecraft had to slew from one target to the next and in some cases, back to the original target in quick succession. This placed enormous strain on the spacecraft ADCS and added complexity to mission operations. Instrument teams 
had to collaborate and coordinate to ensure proper handoff and adequate time for the spacecraft to slew to the next target.

This problem turned out to be common throughout the Cassini Primary Mission. The problem of determining which instrument received priority and when led the mission managers and operators to develop a system of time allocation. These complexities are eliminated by moving the remote sensing instruments to CubeSats. The instruments could still work together to image the same target but could image completely different targets if desired maximizing observation opportunities. As opposed to mounting the instruments on gimbals, moving them to CubeSats has the benefit of reducing the requirements on the Mother greatly simplifying its design.

The fields and particles instruments' methods of collecting data are passive in that they don't require the spacecraft to actively point towards specific target. They are generally left in an "on" state collecting data continuously. Because of their minimal power and data demands as well as not needing to point, there is no benefit of offloading the fields and particles instruments onto separate CubeSat platforms. The exception to this is if one of the fields and particles instruments and its measurements become a primary science objective. If the science objective demanded higher spatial and temporal resolution measurements, a swarm of CubeSats with that instrument could be deployed to take measurements over different locations at the same time or the same location many times in quick succession.

The Juno mission cost was $\$ 1.13$ billion which included the spacecraft development, science instruments, launch services, mission operation, science data processing and relay support [30]. The eight remote sensing CubeSats will likely cost an estimated $\$ 250$ and the architecture's extra four years of mission duration compared to Juno will add another \$416 million. Assuming similar Juno mission costs, the Remote Sensing Swarm architecture will likely cost approximately $\$ 1.8$ billion. The Remote Sensing Swarm will cost just $46 \%$ of the Cassini's price of $\$ 3.95$ billion and still return $80 \%$ of the imaging data and $100 \%$ of the fields and particles instrument data.

The Remote Sensing Swarm architecture is the ideal deep space CubeSat architecture for maximizing scientific return while minimizing mission cost. The architecture reduces carrier spacecraft complexity and 
increases observation opportunities. However, the miniaturized instruments will likely sacrifice spectral range, SNR or resolution. Cassini's science objectives demanded large spectral ranges to ensure as much could be discovered as possible. While the degree of sacrifice could be minimal, the laws of physics dictate sacrifices will be made. Scientists in the future will need to decide if the reduction in mission cost is worth the reduction in data quality. 


\section{Recommendations and Future Work}

\subsection{Recommendations}

The primary driver of complexity of interplanetary missions are the remote sensing instruments. These instruments. These instruments require the spacecraft to be three axis stabilized which increases power demand, propellant usage, and overall complexity of the design and structure. Moving the remote sensing instruments to free flying CubeSats would dramatically reduce the complexity of the Mother spacecraft and therefore the overall mission cost. With the remote sensing instruments removed, the Mother is free to be spin stabilized allowing it to be solar powered. Solar power would further reduce mission costs by eliminating the need for expensive RTGs and with amount of radioactive fuel available, RTGs might not even be a viable power source in the near future.

Although this thesis shows that remote sensing CubeSats could be used for Flagship missions, current instruments do not have the spectral range to perform the wide variety of investigations that Cassini achieved. Narrowing the scope of the mission to be performed is a good way to incorporate CubeSats in the immediate future. The narrow scope allows for tailored and narrow band instrument to be used which will ease instrument design and increase performance. Two possible missions are proposed, but many more exist.

Cassini found active water geysers at the south pole of Enceladus in an area known as the Tiger Stripes [59]. Anytime liquid water is found in the solar system, significant interest is generated for further investigation of the source as evidenced by the proposed Europa Clipper mission to investigate the subsurface oceans on Europa [73]. The Europa Clipper in its current conception doesn't include a lander but a future landing mission is desired [74]. Future exploration of Enceladus will likely go the same way; first a targeted orbiter followed by a lander. To reinforce this idea, the Enceladus Life Finder mission is currently being considered as the next NASA New Frontiers mission [60]. A CubeSat could accompany the Enceladus Life Finder to capture high resolution images of the Tiger Stripe's surface in preparation for a future lander.

Alternatively, a high-resolution imaging CubeSat could get closer to Saturn's rings and image individual ring particles, something Cassini was never able to do. Scientists are still unsure of the age of the rings [75] 
and imaging individual particles to resolve their size, shape and composition would go a long way to determine the rings' age.

This thesis concentrated on existing and near term technologies to show that CubeSats are capable and ready to perform Flagship and high priority science missions. Looking forward to far future technologies, one architecture presents itself that could solve many of the issues that affect the RSS architecture. The Hive concept involves the remote sensing CubeSats using the mother as a docking port. The CubeSats would release from the Mother at the beginning of their 12 hour operation period, conduct their observations, and then return to the Mother to recharge, downlink data, and be escorted through trajectory correction maneuvers. The Hive architecture would dramatically reduce the solar area of the CubeSats as they would only have small solar panels in case the CubeSat failed to dock at the appropriate time. Their primary power source would be the Mother. The propulsion unit would also be smaller, only being required to redock the CubeSat. Because the data collected would be transmitted to the mother while docked, the high power Iris radio would not be needed. These reductions would likely enable the CubeSats to shrink back to a $6 \mathrm{U}$ form factor. A significant amount of work is needed to develop a relative navigation system and docking port for this concept. Power and data would need to transmitted wirelessly because reattaching connectors id unlikely. Commercially available wireless charging ports exist for cellphones but the technology would need to be adapted for high power and the space environment. For data downlink, systems such as Wi-Fi or Bluetooth could be used but again would need to be adapted for the space environment. Relative navigation and maneuvering techniques would also need to be developed to allow the CubeSats to consistently dock and undock to the spinning mother. Additionally, the physical docking attachment and deployer will need to be developed. Much work is required to bring the Hive concept into the realm of feasibility but the concept offers tremendous potential.

While the RSS concept would be the best architecture for replicating Cassini or performing near future missions with CubeSats and existing technology, in the future, the TS architecture and a Hive version of it, offer the potential to develop a standard deep space exploration vehicle. A single, standardized Mother could be developed such that it could travel to many different locations in the solar system and only the CubeSats would be changed for the different destinations. This Deep Space Carrier concept would provide 
a modular, fast and cheap way to explore our solar system, leveraging all the benefits that CubeSats provide.

\subsection{Future Work}

This section outlines topics of study that could further define deep space CubeSat architectures and technologies. The proposed topics are an initial selection that are immediately relevant to the material presented in this thesis.

\subsubsection{Deep Space Deployer (DSD)}

Current deployers are generally simple metal canister that deploy CubeSats soon after launch. The deployers don't provide any thermal or radiation protection and don't supply the CubeSat with any data or power connections. A deep space deployer will need to provide all of these for future interplanetary CubeSats. A deep space deployer will also need to have a reliable method of deploying the CubeSats after multi yearlong cruises. Additionally, a deep space deployer will need to be standardized to simplify design, reduce manufacturing costs and allow for easy interfacing with potential partner institutions. Because interplanetary missions often use gravity assists from the inner planets, the thermal environments found near the Sun need to be considered as thoroughly as the cold case at the destination planet. However, unlike past deep space deployer concepts, the CubeSat is an instrument of the primary mission. This allows the CubeSat and deployer to dictate their placement on the carrier spacecraft potentially easing design considerations.

\subsubsection{Trajectory Analysis and Optimization}

It was assumed for this analysis that the architectures considered could follow both Cassini's trajectories to Saturn and during its tour. The design and optimization of a trajectory for the Remote Sensing Swarm would be a worthwhile investigation.

Alternatively, it would be interesting to see if it was possible for the Mother to use a solar electric propulsion system and what effect it would have on launch mass and mission duration. 


\subsubsection{Huygens Replication}

The Huygens probe was not a part of this analysis but played an important role in the Cassini mission. The design of a miniaturized Huygens probe using CubeSat technology and components would complement this thesis.

\subsubsection{Detailed Spacecraft Design}

While initial designs where offered for the CubeSats, detailed design of any one of the CubeSats would be beneficial to further investigate the technological feasibility of a deep space remote sensing CubeSat. Areas of interest include the thermal design of the CubeSat and the mechanical design of large solar arrays.

\subsubsection{Laser Communication}

Laser communication was not analyzed in this study but a detailed trade between radio frequency and laser communication as well as a detailed laser communication architecture design would be useful. Direct to Earth laser communications for the CubeSats as well as the Mother should be considered. Of interest a trade between adding a laser communication system to the Mother vs. utilizing the RF system the Mother would likely have to perform radio occultation science.

\subsubsection{Inter-Satellite Communication System}

A key technology to enable the Remote Sensing swarm and architectures like it is the Inter-Satellite Communication System. This system would be responsible for relaying data and commands between the Mother and CubeSats and determining the position of the CubeSats. The CubeSats need to stay close to the Mother to ensure the swarm stays together through multiple gravity assists so accurate position knowledge is crucial to avoid collisions. Systems such as Electra that perform similar duties exist but need to be modified for $\mathrm{X}$ band. This system design and the laser communication trade discussed previously would benefit from concurrent investigation.

\subsubsection{Hive Concept}

The Hive concept, as mentioned above, could solve many of the technological challenges that drive the CubeSats to be 12U. having the CubeSats dock to the Mother between operations reduces the size of the propulsion unit, solar panels, and telecommunication system. The Hive concept would allow the CubeSats 
to operate for longer periods of time by reducing the recharge time. by moving the majority of the solar panels to the Mother, the Hive concept could allow CubeSats to operate at locations farther than Saturn.

The CubeSats proposed for the RSS architecture would not be able to generate enough power at Uranus as the solar panels are not big enough and increasing their size is infeasible. Development of a relative navigation system, docking port, data connections and power transfer is required for this concept. 


\section{Conclusion}

The Cassini Primary Mission could be replicated with a CubeSat swarm at a cost of just $\$ 1.8$ billion, or $46 \%$ of Cassini's cost. Remote sensing data would potentially be reduced by $20 \%$ but at a cost savings of 54\%. The feasible architecture developed in this thesis to replicate the Cassini Primary mission also demonstrates that CubeSats have the ability to perform Flagship class missions and reduce costs. While CubeSats can be used for many instruments, they are most cost effective for remote sensing applications.

Much work is needed to further miniaturize instruments and increase their performance and range. The four remote sensing instruments used by Cassini will be difficult to miniaturize because of their large spectral range. These instruments needed the range because they were investigating a new planetary system and wanted to gather as much breadth of knowledge as possible. Narrowing the spectral range would make miniaturizing the instruments easier. CubeSats are limited to a certain size by the CubeSat standard so, at a certain point, the laws of physics prohibit further downsizing of an instrument. Because of their limited power production, CubeSats aren't the best suited for constant or near constant observation. To achieve day- in and day-out observations, multiple of the same CubeSat will be needed to insure continuous coverage while the other CubeSats recharge. Instead, CubeSats are better suited for targeted observations where after flying by the target they can recharge before coming back to it. Specific targets with narrow scientific goals are better for CubeSats as the instruments can more easily be made for the investigation. For the outer planets, including Saturn, CubeSats are ideal for investigating moons as they provide a narrow study area and multi flyby orbits provide enough time for the CubeSats to recharge.

Future flagship missions could use CubeSats for remote sensing applications to dramatically reduce costs, but, range performance will be reduced. Depending on the scientific desires and budgetary constraints, CubeSats can play a major role in future Flagship missions, especially for remote sensing instruments. However, CubeSats are currently better suited for more specific investigations with narrower scope.

CubeSat technology today is best suited for missions with narrow focuses of investigation. With further development of miniaturized instruments, CubeSats could be used on Flagship class and other high priority science missions to reduce costs. CubeSats would best be used for removing the remote sensing instruments from the carrier spacecraft as these instruments are what drive the complexity of the carrier and 
removing them drastically simplifies the carrier. Advances in LILT solar cells and advanced solar panels could extend solar powered spacecraft's reach to Saturn and potentially beyond. In the future, standardized Deep Space Carrier spacecraft could create a modular method of exploring our solar system. The Hive concept, where the CubeSats redock to the Deep Space Carrier, could eliminate many of the power and propulsion problems of using CubeSats for extended periods of time in deep space. 


\section{References}

[1] E. Piazza, "Cassini Solstice Missions FAQs," NASA JPL, [Online]. Available: https://saturnarchive.jpl.nasa.gov/faq/FAQMission/. [Accessed 8 September 2017].

[2] Bureau of Labor Staistics, "Consumer Price Index Inflation Calculator," Bureau of Labor Staistics, [Online]. Available: https://data.bls.gov/cgi-bin/cpicalc.pl. [Accessed 8 September 2017].

[3] Jet Propulsion Laboratory, "Mars Cube One (MarCO)," Jet Propulsion Laboratory, [Online]. Available: https://www.jpl.nasa.gov/cubesat/missions/marco.php. [Accessed 23 October 2017].

[4] NASA Goddard Space Flight Center, "Saturnian Satellite Fact Sheet," NASA Goddard Space Flight Center, 13 October 2015. [Online]. Available: https://nssdc.gsfc.nasa.gov/planetary/factsheet/saturniansatfact.html. [Accessed 12 September 2017].

[5] NASA, "Titan Overview," NASA, [Online]. Available: https://solarsystem.nasa.gov/planets/titan. [Accessed 29 October 2017].

[6] Jet Propulsion Laboratory, "Cassini Mission to Saturn," NASA JPL, 1 February 2017. [Online]. Available: https://saturn.jpl.nasa.gov/. [Accessed 6 February 2017].

[7] NASA, "Galileo Mission to Jupiter," Jet Propulsion Laboratory, [Online]. Available: https://www.jpl.nasa.gov/news/fact_sheets/galileo.pdf. [Accessed 29 October 2017].

[8] Jet Propulsion Laboratory, "Cassini The Grand Finale," [Online]. Available: https://saturn.jpl.nasa.gov/resources/67/. [Accessed 10 September 2017].

[9] F. a. S. F. Peralta, "Cassini Interplanetary Trajectory Design," Contro Eng. Practice, vol. 3, no. 11, pp. 1603-1610, 1995.

[10] A. Y. Lee and G. Hanover, "Cassini Spacecraft Attitude Control System Flight Performance," in AIAA Guidance, Navigation, and Control Conference and Exhibit, San Fransisco, 2005.

[11] Jet Propulsion Laboratory, "Cassini Mission Plan," May 2002. [Online]. Available: http://caps.space.swri.edu/caps/tourInfo/Documents/MP_N.pdf. [Accessed 11 October 2017].

[12] G. L. Bennett, "Space Nuclear Power: Opening the Final Frontier," in 4th International Energy Conversion Engineering Conference and Exhibit (IECEC), San Diego, California, 2006.

[13] NASA, "Radioisotope Power Systems," NASA, 13 July 2013. [Online]. Available: https://solarsystem.nasa.gov/rps/cassini.cfm. [Accessed 11 September 2017].

[14] J. Taylor, L. Sakamoto and C.-J. Wong, "Cassini Telecommunications," January 2002. [Online]. Available: https://descanso.jpl.nasa.gov/DPSummary/Descanso3--Cassini2.pdf. [Accessed 10 October 2017]. 
[15] M. W. Leeds, R. N. Eberhadt and R. L. Berry, "Development of the Cassini Spacecraft Propulsion Subsystem," in 32nd AIAA/ASME/SAE/ASEE Joint Propulsion Conference, Lake Buena Vista, 1996.

[16] C. C. Porco, "Cassini Imaging Science: Instrument Characteristics and Anticipated Scientific Investigations at Saturn," Space Science Reviews, vol. 115, no. 1-4, pp. 363-497, 2004.

[17] R. H. Brown, "The Cassini Visual and Infrared Mapping Spectrometer (VIMS) Investigation," Space Science Reviews, vol. 115, no. 1-4, pp. 111-168, 2004.

[18] F. M. Flasar, "Exploring the Saturn System in the Thermal Infrared: The Composite Infrared Spectrometer," Space Science Reviews, vol. 115, no. 1-4, pp. 169-297, 2004.

[19] L. Esposito, "The Cassini Ultraviolet Imaging Spectrograph Investigation," Space Science Reviews, vol. 115, no. 1-4, pp. 299-361, 2004.

[20] C. Elachi, "Radar: The Cassini Titan Radar Mapper," Space Science Reviews, vol. 115, no. 1-4, pp. 71-110, 2004.

[21] D. T. Young, "Cassini Plasma Spectrometer Investigation," Space Science Reviews, vol. 114, no. 14, pp. 1-112, 2004.

[22] R. Srama, "The Cassini Cosmic Dust Analyzer," Space Science Reviews, vol. 114, no. 1-4, pp. 465$518,2004$.

[23] J. H. W. Jr, "The Cassini Ion and Neutral Mass Spectrometer (INMS) Investogation," Space Science Reviews, vol. 114, no. 1-4, pp. 113-231, 2004.

[24] M. K. Dougherty, "The Cassini Magnetic Field Investigation," Space Science Reviews, vol. 114, no. 1-4, pp. 331-383, 2004.

[25] S. M. Krimigis, "Magnetosphere Imaging Instrument (MIMI) on the Cassini Mission to Saturn/Titan," Space Science Reviews, vol. 114, no. 1-4, pp. 233-329, 2004.

[26] D. A. Gurnett, "The Cassini Radio and Plasma Wave Investigation," Space Science Reviews, vol. 114, no. 1-4, pp. 395-463, 2004.

[27] A. J. Kliore, "Cassini Radio Science," Space Science Reviews, vol. 115, no. 1-4, pp. 1-70, 2004.

[28] J. Foust, "NASA seeks cost-cutting changes in design of WFIRST mission," SpaceNews, 20 October 2017. [Online]. Available: http://spacenews.com/nasa-seeks-cost-cutting-changes-in-design-ofwfirst-mission/. [Accessed 29 October 2017].

[29] T. D. Goodson, D. L. Gray, Y. Hahn and F. Peralta, "Cassini Maneuver Experience: Launch and Early Cruise," in Guidance, Navigation, and Control Conference and Exhibit, Boston, 1998.

[30] NASA Jet Propulsion Laboratory, "Juno Press Kit," [Online]. Available: https://www.jpl.nasa.gov/news/press_kits/juno/pdf/juno-hires.pdf. [Accessed 12 October 2017]. 
[31] Moog-ISP, "Moog Upper Stage Engines," 2013. [Online]. Available: http://www.moog.com/literature/Space_Defense/Spacecraft/Propulsion/Upper_Stage_Engines_Rev_ 0913.pdf. [Accessed 12 October 2017].

[32] T. Freeman, "Deep Space CubeSats and Nanosats at JPL," in iCubeSat, Cambridge, 2017.

[33] OneWeb, "OneWeb Solution," OneWeb, 2017. [Online]. Available: http://www.oneweb.world/\#solution. [Accessed 17 October 2017].

[34] J. Wenz, "All the NASA Planetary Missions That Are Currently Active," Popular Mechanics, 14 July 2015. [Online]. Available: http://www.popularmechanics.com/space/a16444/nasa-activemissions/. [Accessed 17 October 2017].

[35] NASA, "Deep Space Network," [Online]. Available: https://deepspace.jpl.nasa.gov/files/dsn/820100\%20F.pdf. [Accessed 12 September 2017].

[36] R. Staehle and a. et, "Interplanetary CubeSats: Opening the Solar System to a Broad Community at Lower Cost," 8 December 2012. [Online]. Available: https://www.nasa.gov/pdf/716078main_Staehle_2011_PhI_CubeSat.pdf. [Accessed 3 October 2017].

[37] A. Boca and e. al, "Advanced-Architecture High-Efficiency Solar Cells," Jet Propulsion Laboratory, Pasadena, 2017.

[38] S. Clark, "Prototype solar array jettisoned as Dragon capsule prepares for trip home," SpaceFlight Now, 30 June 2017. [Online]. Available: https://spaceflightnow.com/2017/06/30/prototype-solararray-jettisoned-as-dragon-capsule-prepares-for-trip-home/. [Accessed 18 October 2017].

[39] Jet Propulsion Laboratory, "Cassini, Spacecraft Navigation," NASA Jet Propulsion Laboratory, [Online]. Available: https://saturn.jpl.nasa.gov/mission/spacecraft/navigation/. [Accessed 9 October 2017].

[40] NASA, "Juno's Solar Cells Ready to Light Up Jupiter Mission," NASA, 15 June 2011. [Online]. Available: https://www.nasa.gov/mission_pages/juno/launch/Juno_solarpower.html. [Accessed 23 October 2017].

[41] CubeSat, "Developer Resources," 20 April 2016. [Online]. Available: https://static1.squarespace.com/static/5418c831e4b0fa4ecac1bacd/t/573fa2fee321400346075f01/146 3788288448/6U_CDS_2016-05-19_Provisional.pdf. [Accessed 10 October 2017].

[42] Planetary Systems Corporation, "Canisterized Satellite Dispenser Payload Specifications," 4 August 2017. [Online]. Available: http://www.planetarysystemscorp.com/web/wpcontent/uploads/2017/08/2002367E-Payload-Spec-for-3U-6U-12U-27U.pdf. [Accessed 10 October 2017].

[43] Planetary Systems Corp, "Canisterized Satellite Dispenser Data Sheet," 4 August 2017. [Online]. Available: http://www.planetarysystemscorp.com/web/wp-content/uploads/2017/08/2002337E-CSDData-Sheet.pdf. [Accessed 13 September 2017]. 
[44] R. Staehle and e. al, "Interplanetary CubeSats: Opening the Solar System to a Broad Community at Lower Cost," 8 December 2012. [Online]. Available: https://www.nasa.gov/pdf/716078main_Staehle_2011_PhI_CubeSat.pdf. [Accessed 3 October 2017].

[45] United Launch Alliance, "Rocket Builder," United Launch Alliance, 2016. [Online]. Available: https://www.rocketbuilder.com/start/configure. [Accessed 23 October 2017].

[46] S. Clark, "ULA needs commercial customers to close Vulcan rocket business case," SpaceFlight Now, 22 April 2016. [Online]. Available: https://spaceflightnow.com/2015/04/22/ula-needscommercial-business-to-close-vulcan-rocket-business-case/. [Accessed 23 October 2017].

[47] NASA Goddard Space Flight Center, "Saturn Fact Sheet," NASA Goddard Space Flight Center, 23 December 2016. [Online]. Available: https://nssdc.gsfc.nasa.gov/planetary/factsheet/saturnfact.html. [Accessed 12 September 2017].

[48] NASA, "JWST Launch," NASA Goddard Space Flight Center, [Online]. Available: https://jwst.nasa.gov/launch.html. [Accessed 23 October 2017].

[49] NASA, "NASA Space Launch System's First Flight to Send Small Sci-Tech Satellites Into Space," NASA, 2 February 2016. [Online]. Available: https://www.nasa.gov/press-release/nasa-spacelaunch-system-s-first-flight-to-send-small-sci-tech-satellites-into-space. [Accessed 23 October 2017].

[50] Malin Space Science Systems, "ECAM-C50," [Online]. Available: http://www.msss.com/brochures/c50.pdf. [Accessed 25 October 2017].

[51] Blue Canyon Technologies, "Attitude Determination Control Systems," 2017. [Online]. Available: http://bluecanyontech.com/wp-content/uploads/2017/07/DataSheet_ADCS_08_F.pdf. [Accessed 24 October 2017].

[52] T. Imken, J. Castillo-Rogez, Y. He, J. Baker and A. Marinan, "CubeSat Flight System Development for Enabling Deep Space Science," in IEEE Aerospace Conference, 2017.

[53] eoPortal, "Lunar IceCube," eoPortal, [Online]. Available:

https://directory.eoportal.org/web/eoportal/satellite-missions///lunar-icecube. [Accessed 24 October 2017].

[54] Aerojet Rocketdyne, "MPS-130 Performance Data," [Online]. Available: http://www.rocket.com/files/aerojet/documents/CubeSat/MPS-130\%20data\%20sheet\%20crop.pdf. [Accessed 25 October 2017].

[55] R. Mukai and a. et, "Juno Telecommunicationd," October 2012. [Online]. Available: https://descanso.jpl.nasa.gov/DPSummary/Juno_DESCANSO_Post121106H--Compact.pdf. [Accessed 25 October 2017].

[56] NASA, "Deep Space Network: Iris Radio," [Online]. Available: https://deepspace.jpl.nasa.gov/files/dsn/Brochure_IrisV2_201507.pdf. [Accessed 12 September 2017]. 
[57] D. Gilmore, Spacecraft Thermal Control Handbook, Volume I: Fundamental Technologies, AIAA, 2002.

[58] E. Wertheimer, L. Berthoud and M. Johnson, "PocketRTG - a CubeSat scale radioisotope," in iCubeSatWorkshop, London, 2015.

[59] NASA, "Cassini Finds Global Ocean in Saturn's Moon Enceladus," NASA, 15 September 2015. [Online]. Available: https://www.nasa.gov/press-release/cassini-finds-global-ocean-in-saturns-moonenceladus. [Accessed 25 October 2017].

[60] M. L. Cable and e. al, "Enceladus Life Finder:," 2016. [Online]. Available: https://trs.jpl.nasa.gov/bitstream/handle/2014/45905/15-4603_A1b.pdf?sequence=1. [Accessed 25 October 2017].

[61] H. B. Garrett, J. M. Ratliff and R. W. Evans, "Saturn Radiation Model," NASA Jet Propulsion Laboratory, 2005.

[62] M. A. Noca and K. J. Hack, "Results of the JIMO Follow-on Destinations Parametric Studies," NASA Jet Propulsion Laboratory, Pasadena, 2005.

[63] M. Moharram and A. Kishk, "Optically Transparent Reflectarray Antenna Design Integrated with Solar Cell," IEEE Transactions on Antennas and Propogation, vol. 64, no. 5, pp. 1700-1712, 2016.

[64] EaglePicher Technologies, "55Ah High Rate Lithium Ion Cell," [Online]. Available: https://www.eaglepicher.com/sites/default/files/NCP55-4\%20APPROVED.pdf. [Accessed 27 October 2017].

[65] M. Janssen and a. et, "MWR: Microwave Radiometer for the Juno Mission to Jupiter," Space Science Review, pp. 1-47, 27 March 2017.

[66] A. Adriani and a. et, "JIRAM: the Jovian Infrared Auroral Mapper," Space Science Reviews, pp. 154, 01 October 2014.

[67] J. E. P. Connerney and a. et, "The Juno Magnetic Field Investigation," Space Science Reviews, pp. 1100, 14 February 2017.

[68] D. J. McComas and a. et, "The Jovian Auroral Distributions Expirement (JADE) on the Juno Mission to Jupiter," Space Science Reviews, pp. 1-97, 25 May 2013.

[69] B. H. Mauk and a. et, "The Jupiter Energetic Particle Detector Instrument (JEDI) Investigation for the Juno Mission," Space Science Reviews, pp. 1-58, 26 November 2013.

[70] W. S. Kurth and a. et, "The Juno Waves Investigation," Space Science Reviews, pp. 1-46, 10 July 2017.

[71] G. R. Gladstone and a. et, "The Ultraviolet Spectrograph on NASA's Juno Mission," Space Science Reviews, pp. 1-27, 25 March 2014. 
[72] C. J. Hansen and a. et, "Junocam: Juno's Outreach Camera," Space Science Reviews, pp. 1-32, 23 August 2014.

[73] Jet Propulsion Laboratory, "Europa Clipper," Jet propulsion Laboratory, [Online]. Available: https://www.jpl.nasa.gov/missions/europa-clipper/. [Accessed 30 October 2017].

[74] NASA, "NASA Receives Science Report on Europa Lander Concept," NASA, 8 February 2017. [Online]. Available: https://www.nasa.gov/feature/nasa-receives-science-report-on-europa-landerconcept. [Accessed 30 October 2017].

[75] J. Amos, "Cassini hints at young age for Saturn's rings," BBC, 30 August 2017. [Online]. Available: http://www.bbc.com/news/science-environment-41091333. [Accessed 31 October 2017 ].

[76] NASA, "Solar System Exploration," [Online]. Available: https://solarsystem.nasa.gov/docs/Final_RHU_Fact_Sheet_2016_5-26-16.pdf. [Accessed 11 September 2017].

[77] SRI International, "60-Foot (18.3-Meter) Parabolic Reflector Antenna Dish," 2016. [Online]. Available: https://www.sri.com/60-foot-parabolic-reflector-antenna-dish. [Accessed 12 September 2017].

[78] D. Gray and Y. Hahn, "Maneuver Analysis of the Cassini Mission," in AIAA Guidance, Navigation and Control Conference, 1995.

[79] N. Strange, T. Goodson and Y. Hahn, "Cassini Tour Redesign fothe Huygens Mission," in AIAA/AAS Astrodymics Specialist Conference and Exhibit, Monterey, 2002.

[80] R. E. Hodges, "ISARA Integrated Solar Array Reflectarray," in Small Satellite Conference, Logan, 2013.

[81] M. Stender and C. Lohgry, "Extending Rideshare: Mission Case Studies Using Propulsive ESPA," in 31st Space Symposium, Colorado Springs, 2015.

[82] NASA, "NASA Launch Services Program Launch Vehicle Performance Website," 20 July 2015. [Online]. Available: https://elvperf.ksc.nasa.gov. [Accessed 13 September 2017].

[83] S. T. Cruz, "National Aeronautics and Space Administration Transition Authorization Act of 2017," 21 March 2017. [Online]. Available: https://www.congress.gov/bill/115th-congress/senatebill/442/text. [Accessed 6 October 2017].

[84] D. Leone, "U.S. Plutonium Stockpile Good for Two More Nuclear Batteries after Mars 2020," SpaceNews, 11 March 2015. [Online]. Available: http://spacenews.com/u-s-plutonium-stockpilegood-for-two-more-nuclear-batteries-after-mars-2020/. [Accessed 23 October 2017].

[85] NASA Jet Propulsion Laboratory, "Cassini Mission to Saturn," 2004. [Online]. Available: https://www.jpl.nasa.gov/news/fact_sheets/cassini.pdf. [Accessed 29 October 2017]. 
Appendix

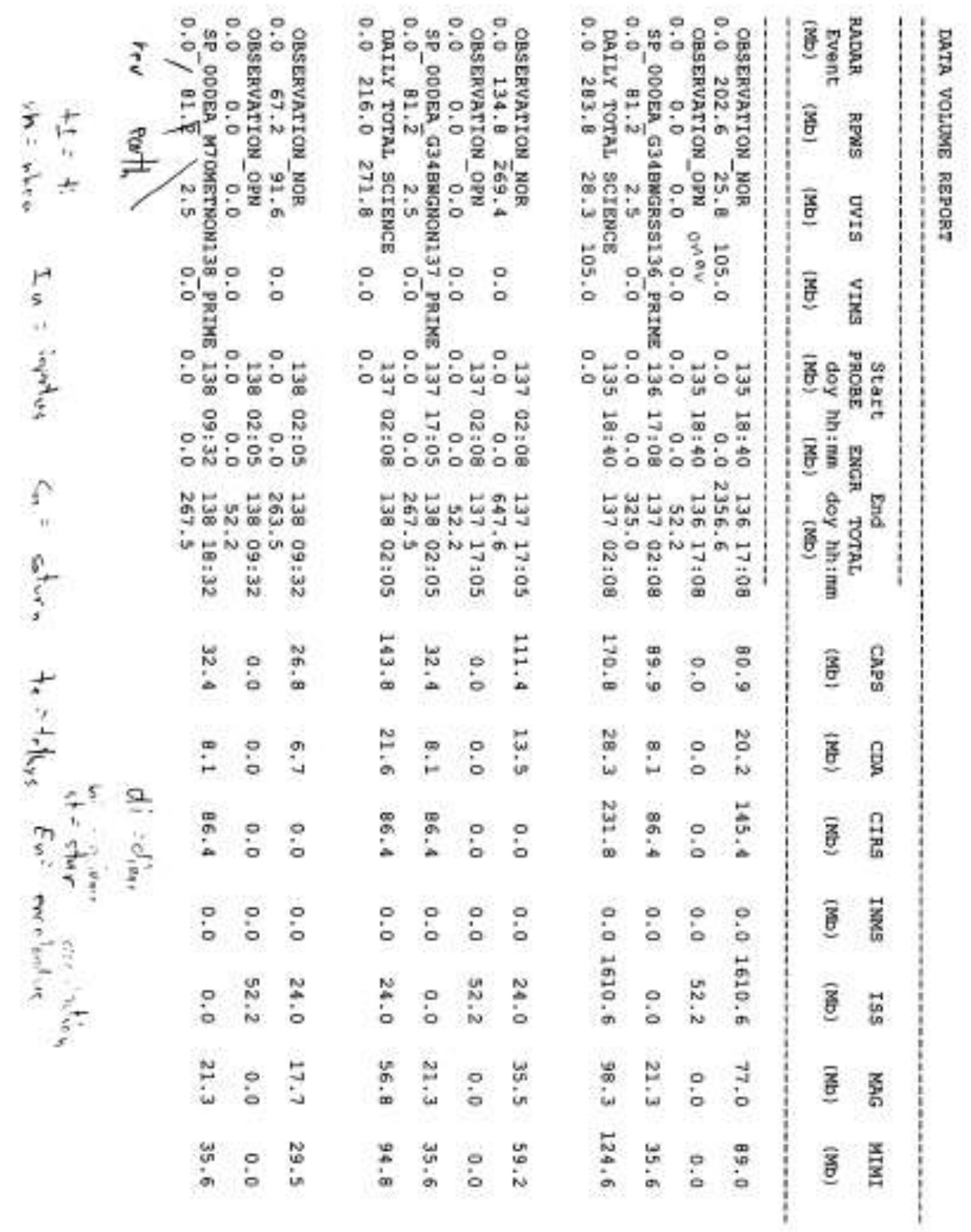



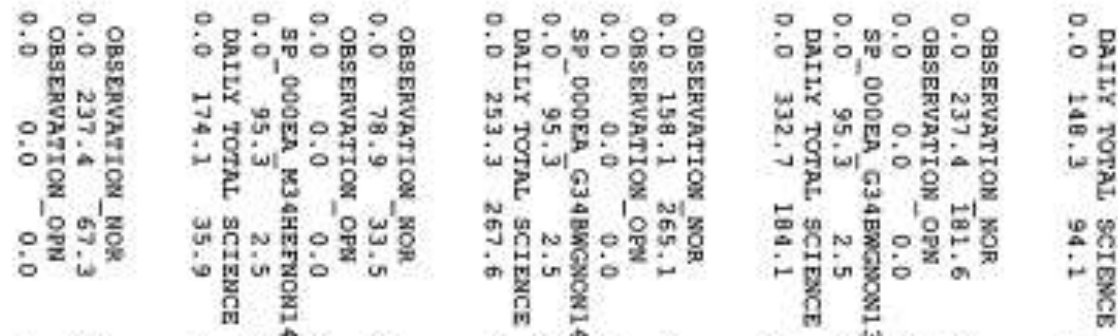

$\therefore: 0$ : 0 桨:

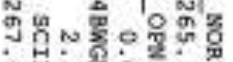

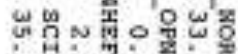

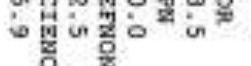

苚

峃

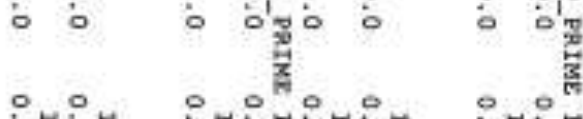

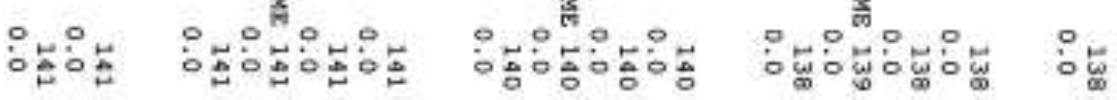

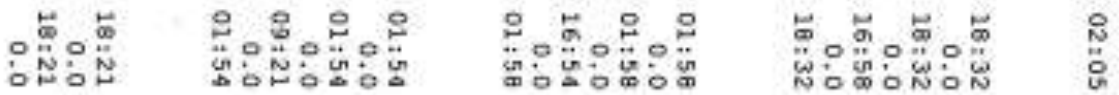

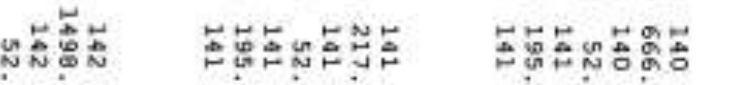

in

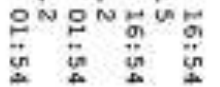

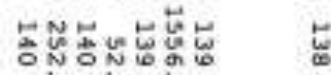

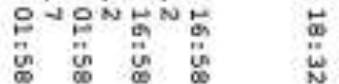

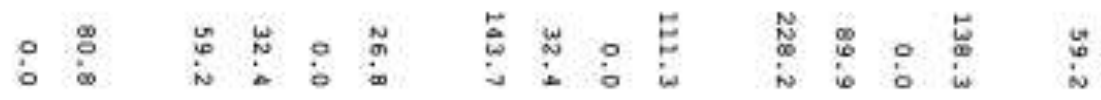

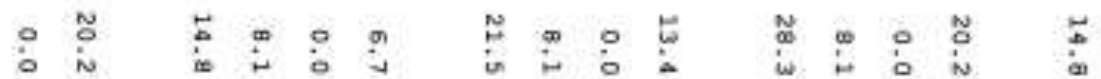

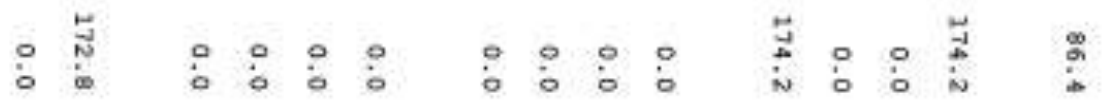

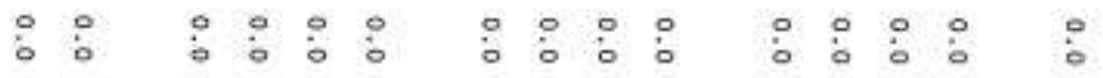

N

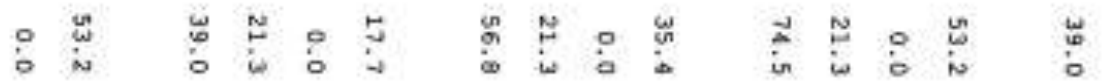

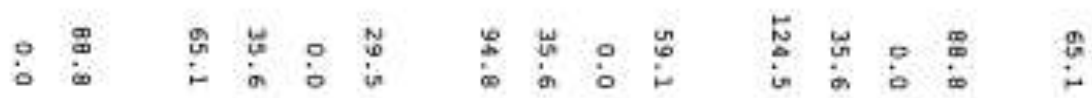




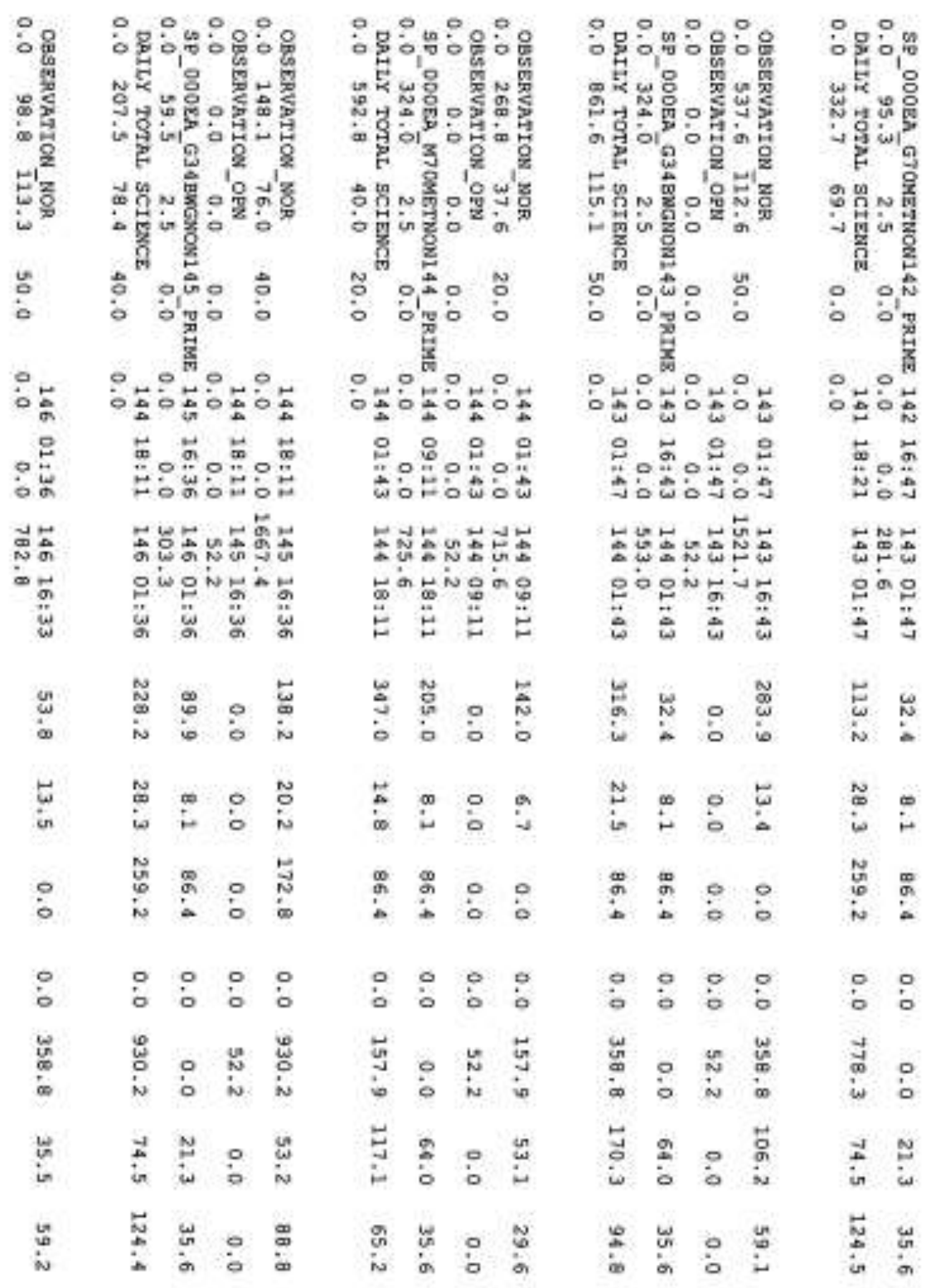




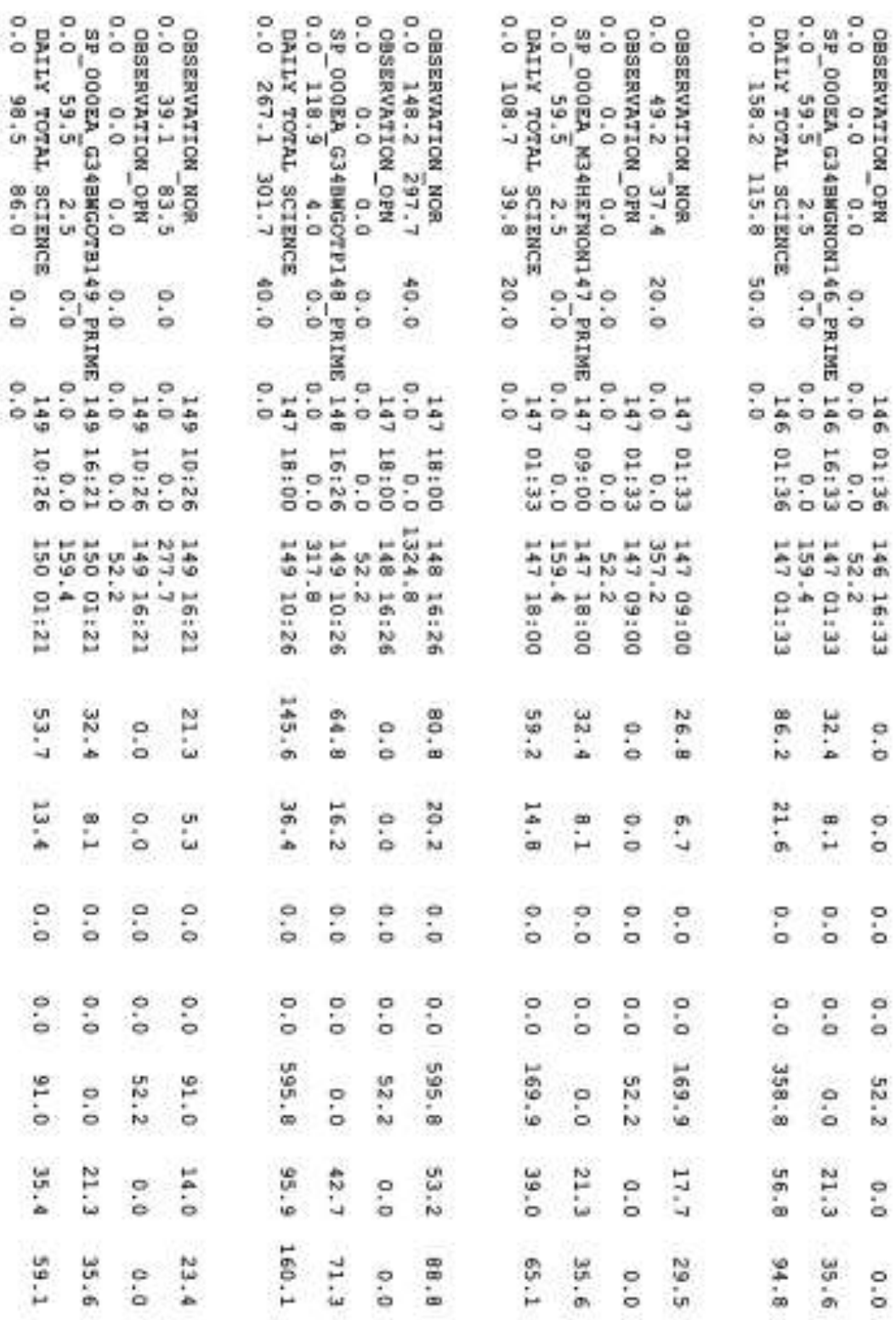




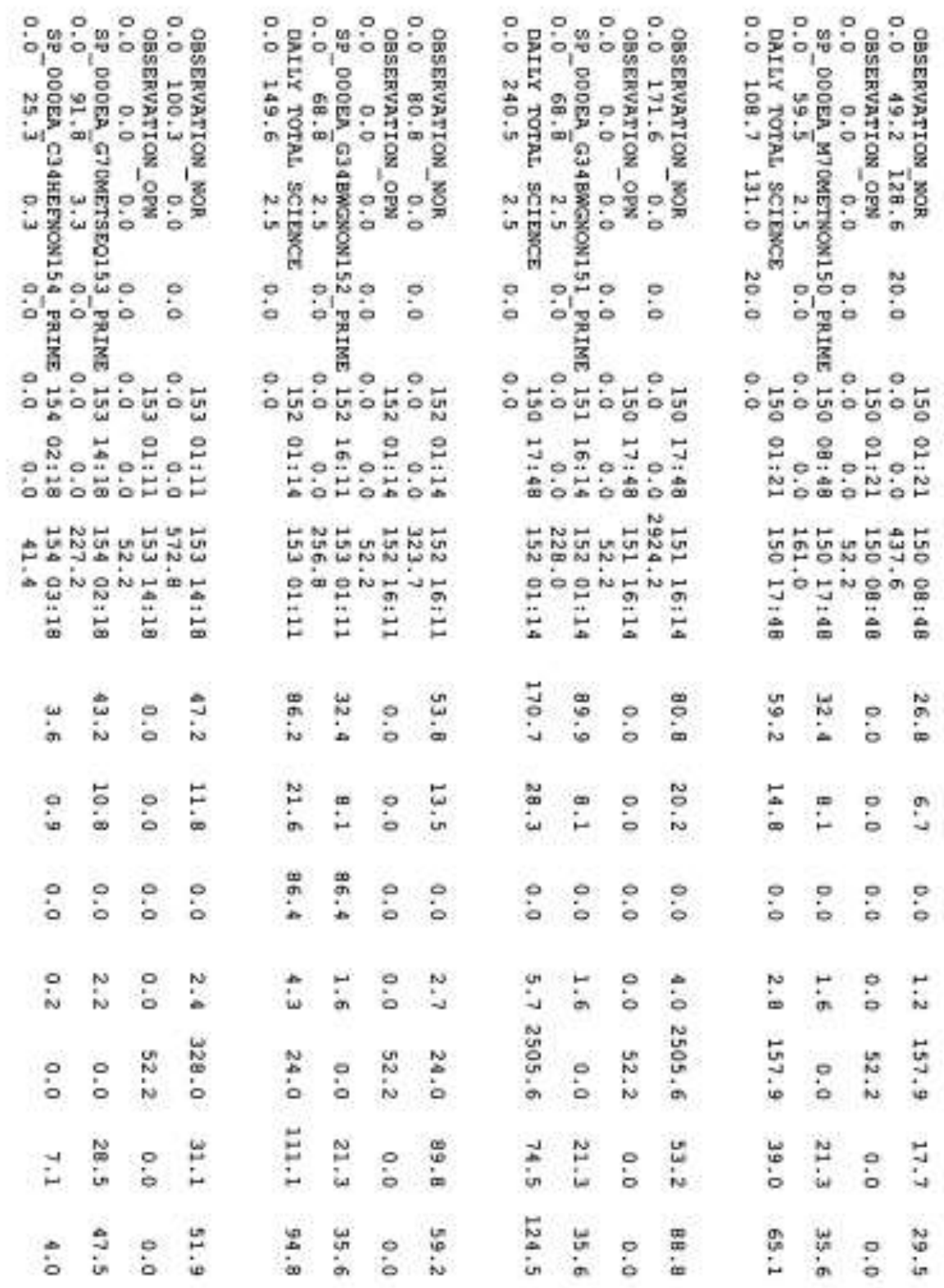




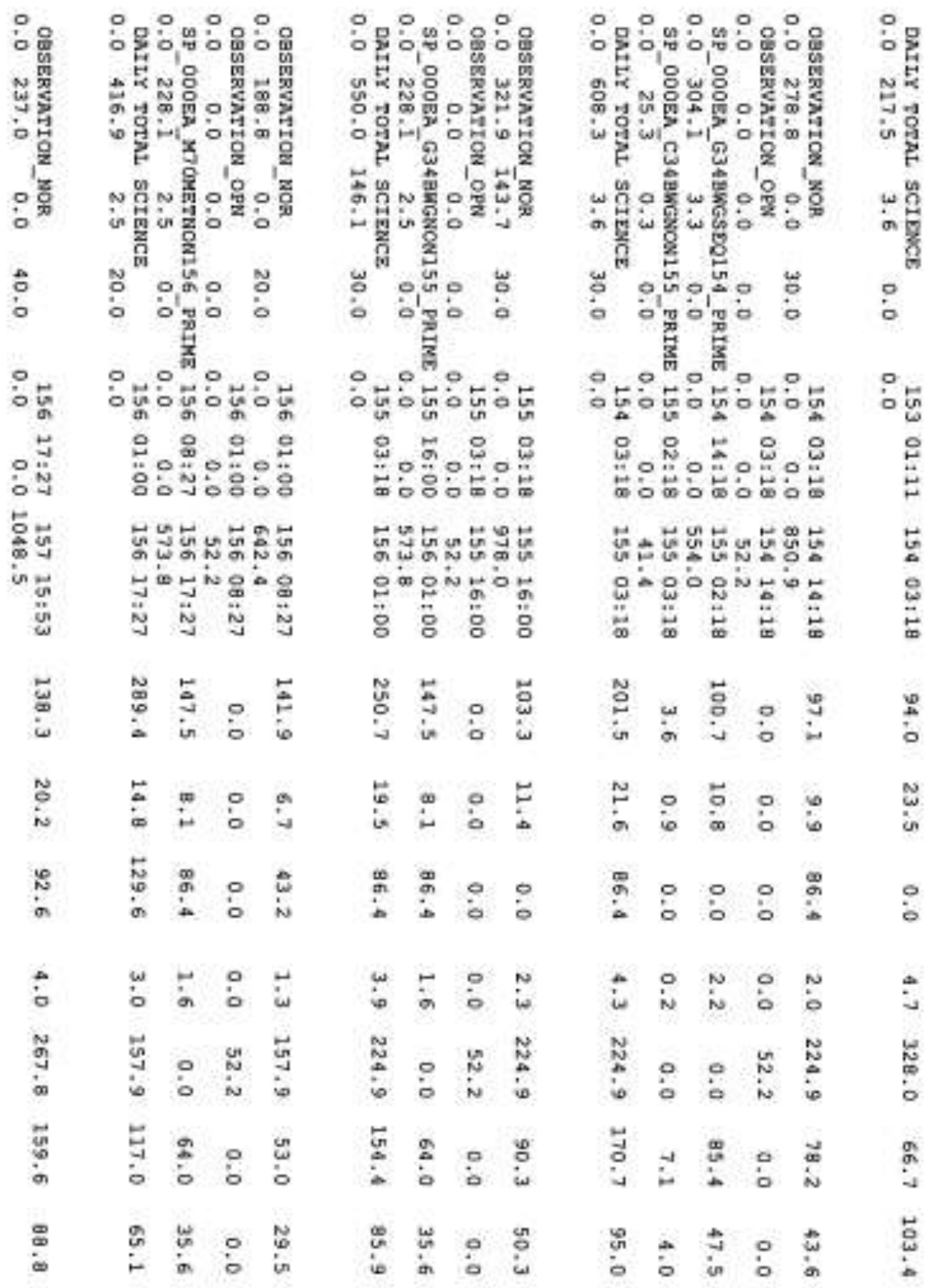




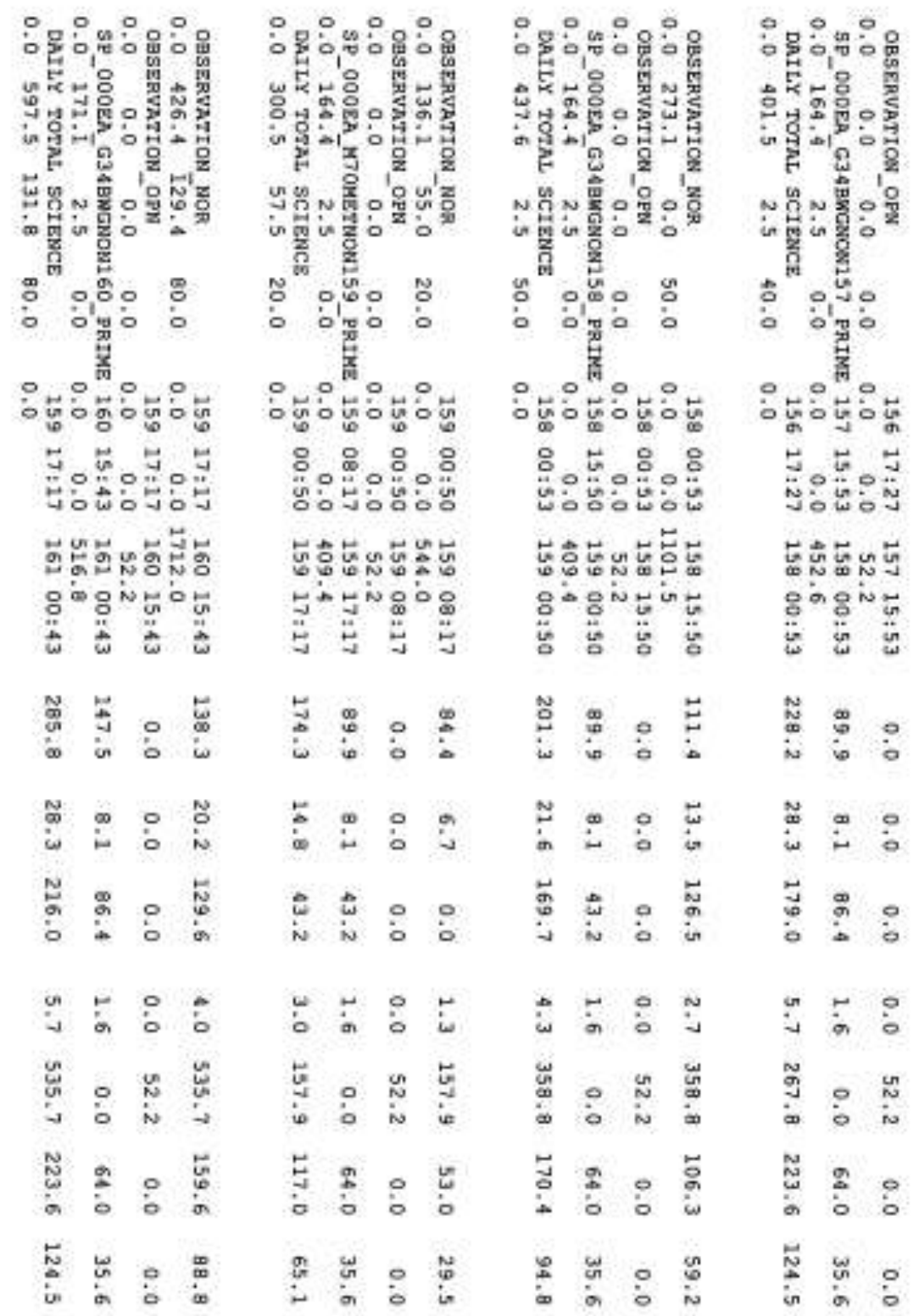



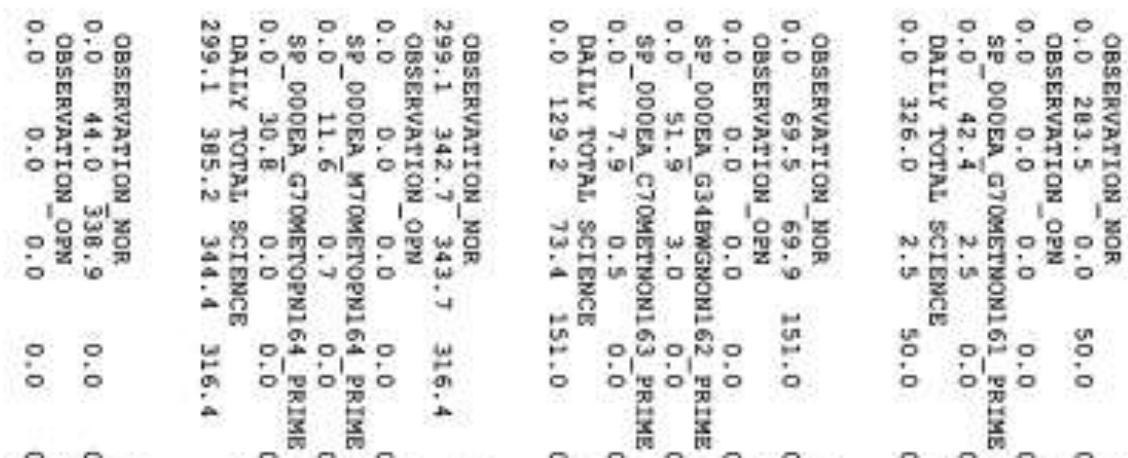

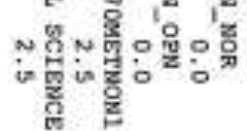

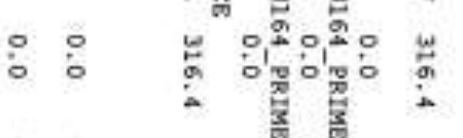

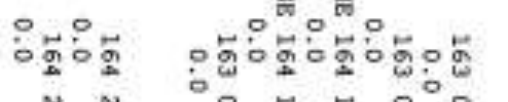

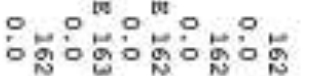

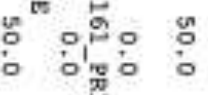

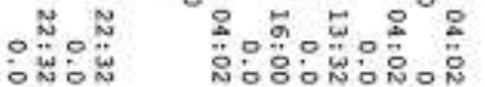

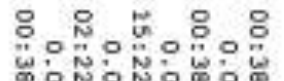

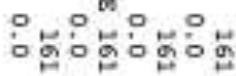

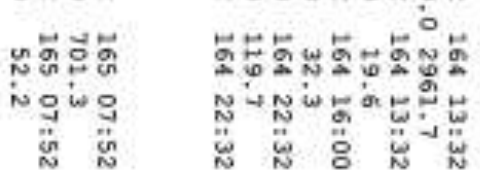

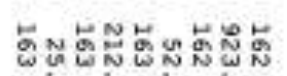

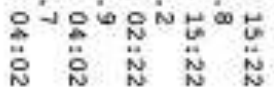

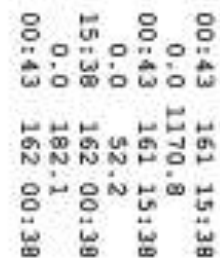

$\circ \underset{\omega}{\omega}$ is

累

¿ $\vdots$ b in

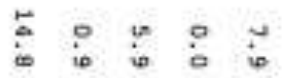

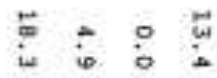

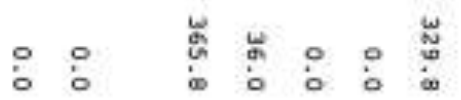

蔡品

苦

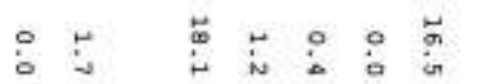

iิ

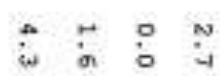

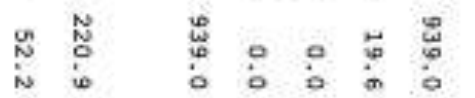

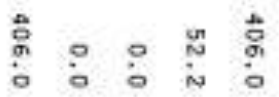

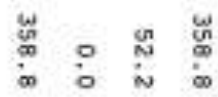

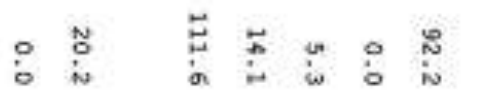

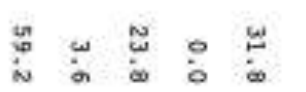

芯:

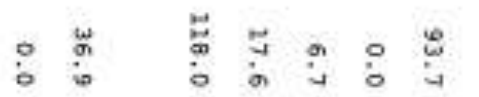

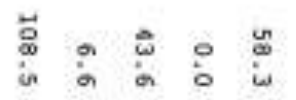

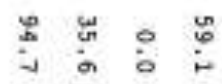




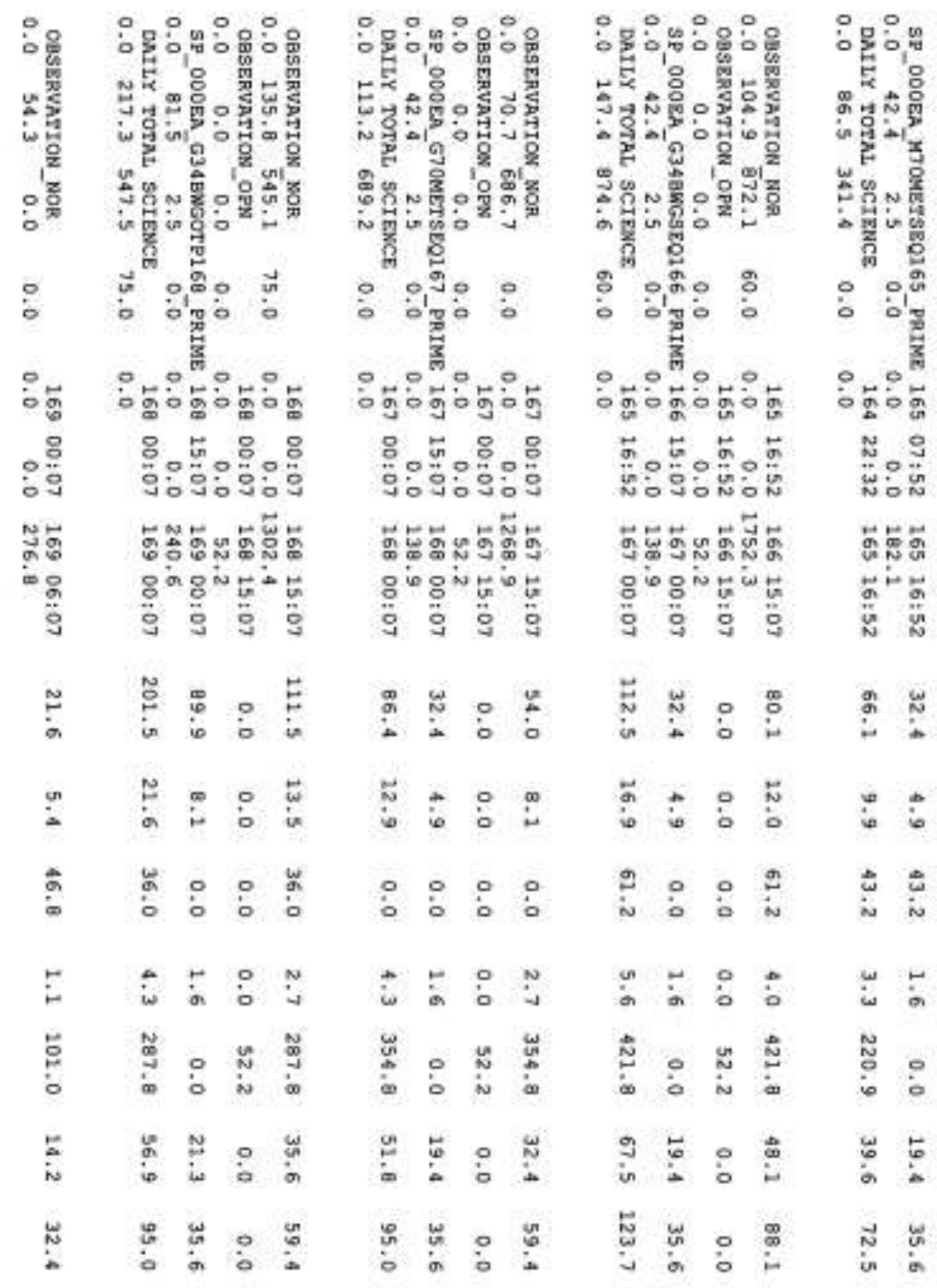




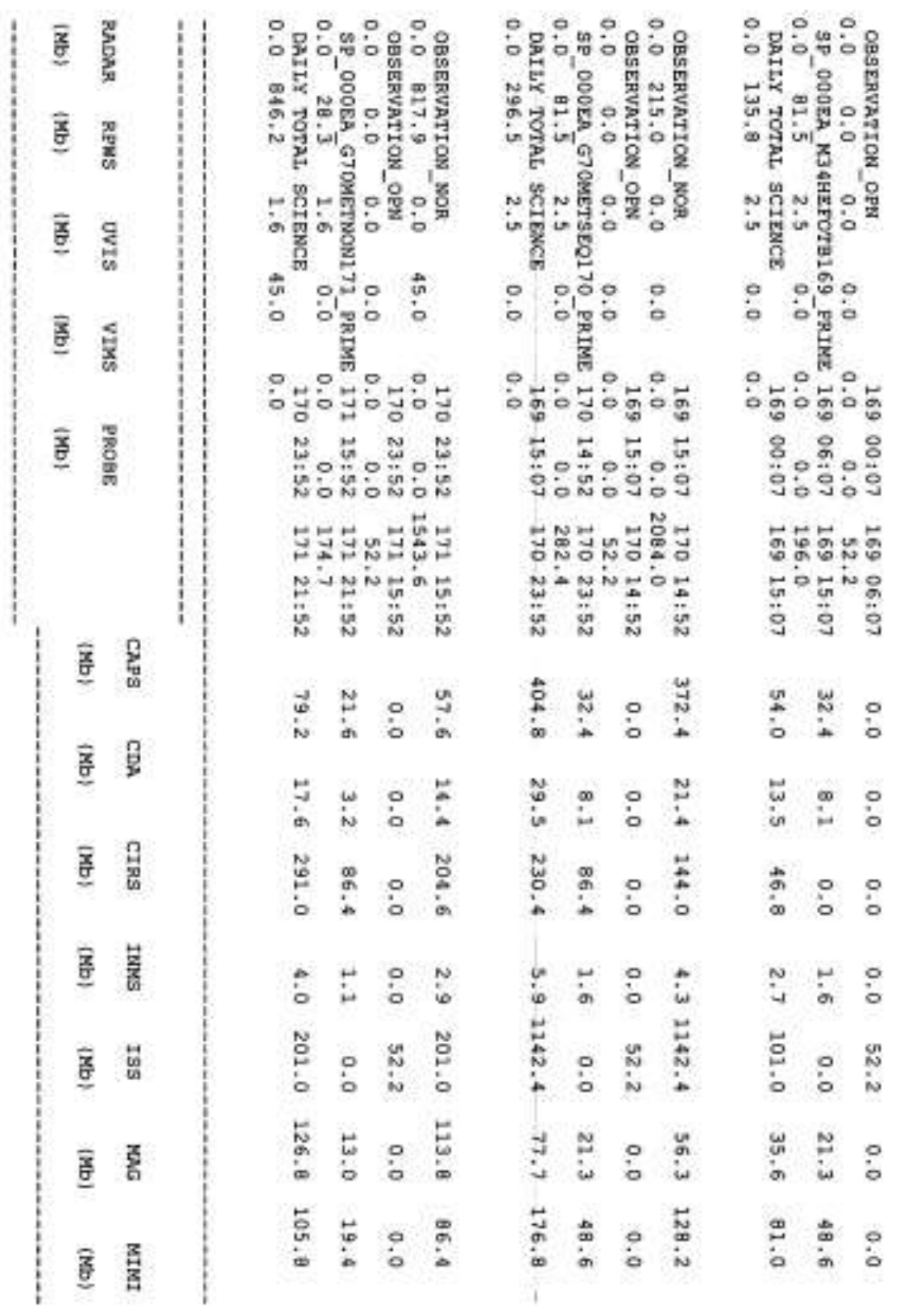




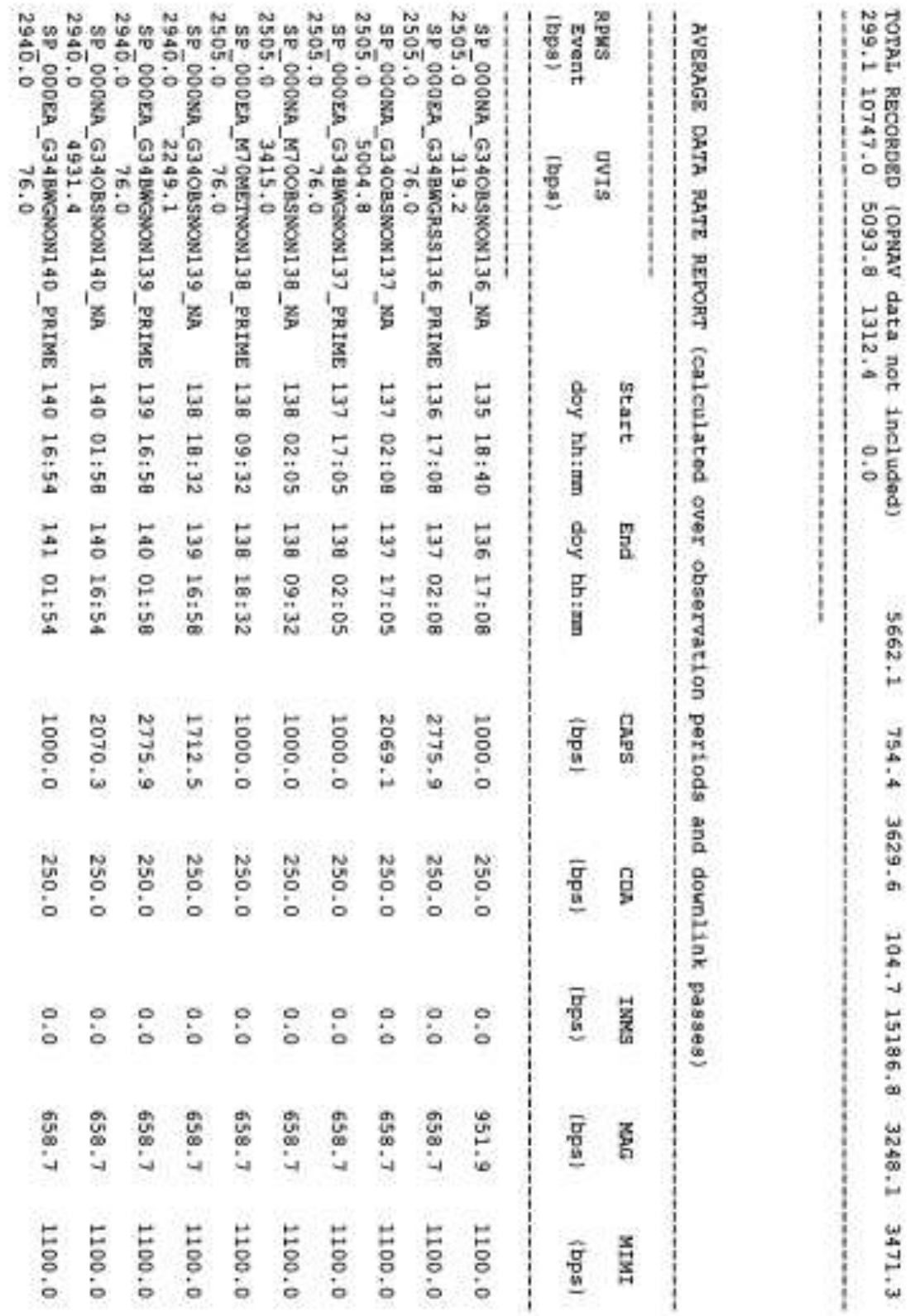

